\title{
Grafos e aplicações de Gauss estáveis
}

\author{
Flavio Henrique de Oliveira
}



Data de Depósito:

Assinatura:

\section{Flavio Henrique de Oliveira}

\section{Grafos e aplicações de Gauss estáveis}

Dissertação apresentada ao Instituto de Ciências
Matemáticas e de Computação - ICMC-USP, como
parte dos requisitos para obtenção do título de
Mestre em Ciências - Matemática. VERSÃO
REVISADA

Área de Concentração: Matemática

Orientadora: Profa. Dra. Ana Claudia Nabarro 
Ficha catalográfica elaborada pela Biblioteca Prof. Achille Bassi e Seção Técnica de Informática, ICMC/USP, com os dados fornecidos pelo(a) autor(a)

Oliveira, Flavio Henrique de

$048 \mathrm{~g}$ Grafos e aplicações de Gauss estáveis / Flavio Henrique de Oliveira; orientadora Ana Claudia Nabarro. -- São Carlos, 2016.

$111 \mathrm{p}$.

Dissertação (Mestrado - Programa de Pós-Graduação em Matemática) -- Instituto de Ciências Matemáticas e de Computação, Universidade de São Paulo, 2016.

1. Grafos de aplicações de Gauss estáveis. I. Nabarro, Ana Claudia, orient. II. Título. 


\section{Flavio Henrique de Oliveira}

\section{Graphs and stable Gauss applications}

\begin{abstract}
Master dissertation submitted to the Instituto de Ciências Matemáticas e de Computação - ICMCUSP, in partial fulfillment of the requirements for the degree of the Master Program in Mathematics. FINAL VERSION
\end{abstract}

Concentration Area: Mathematics

Advisor: Profa. Dra. Ana Claudia Nabarro

\section{USP - São Carlos}

August 2016 

"Paradoxo, humor e mudança."

O caminho do guerreiro pacífico 


\section{Agradecimentos}

Primeiramente, agradeço a Deus por ter me dado esta oportunidade.

Agradeço aos meus pais, pela base sólida que me deu força para encarar a vida e pelo seu imenso amor.

Agradeço à professora Ana Claudia Nabarro, os meus mais sinceros agradecimentos pela sua orientação, dedicação e por ter sido tão importante no meu crescimento profissional.

Agradeço à professora Luciana de Fátima Martins por ter me dado um empurrão para que eu tivesse essa oportunidade.

Aos professores e funcionários do ICMC por tornarem a minha jornada por aqui possível e pelo trabalho dedicado.

À CAPES, pelo suporte financeiro.

Enfim, agradeço a todos que colaboraram com a realização deste sonho. 


\section{Índice}

Resumo ....................... ix

Abstract ........................... xi

Introdução . . . . . . . . . . . . . . . . . . . . . . . . . . . 1

1 Resultados preliminares $\quad 3$

1.1 Característica de Euler . . . . . . . . . . . . . . . . . . . . . 3

1.1.1 Complexos . . . . . . . . . . . . . . . . . . . . 4

1.1.2 Superfícies e característica de Euler . . . . . . . . . . . . . 7

1.2 Grafos e Matrizes . . . . . . . . . . . . . . . . . . . . . . . . . . . . . 12

1.2.1 Ciclos independentes . . . . . . . . . . . . . . . . . . . . 14

1.2 .2 Grafos bipartidos . . . . . . . . . . . . . . . . 17

1.3 Preliminares da teoria de Singularidades . . . . . . . . . . . . . 18

1.3.1 Aplicações estáveis e aplicação de Gauss . . . . . . . . . . . . . 18

$1.3 .2 \quad$ Jatos . . . . . . . . . . . . . . . . . . . . . 20

1.3.3 A topologia $C^{\infty}$ de Whitney . . . . . . . . . . . . . . . . . 21

1.3.4 Conjunto singular e contorno aparente . . . . . . . . . . . . 23

1.3.5 Grau de aplicações . . . . . . . . . . . . . . . . . . . 26

1.3.6 Aplicação de Gauss e superfícies . . . . . . . . . . . . . . . . . . 27

1.4 Germes de aplicações diferenciáveis . . . . . . . . . . . . . . . . . . . 30

1.4.1 Desdobramento . . . . . . . . . . . . . . . . . . . . . . 31

1.4.2 Classificação dos germes de funções . . . . . . . . . . . . . . . . 32

1.4.3 Classificação dos germes $\mathbb{R}^{2} \rightarrow \mathbb{R}^{2} \ldots \ldots \ldots \ldots . \ldots \ldots$

1.4.4 Singularidades de Morin . . . . . . . . . . . . . . . . . . 33 
1.5 Teoria da catástrofe de Thom-Zeeman . . . . . . . . . . . . . . . . . 34

2 Aplicações de Gauss, transições e cirurgias 35

2.1 Forma de Monge e familia de funções altura de uma superfície . . . . . . . 36

2.2 Aplicação Catástrofe da família de funções altura . . . . . . . . . . . . . . 37

2.3 Sobre a classificação local das singularidades da função altura . . . . . . . 42

2.4 Transições locais . . . . . . . . . . . . . . . . . . . . . . . 45

2.4.1 Transição $A_{3}$ : lábios/bicos . . . . . . . . . . . . . . . 46

2.4 .2 Transição $A_{4}$. . . . . . . . . . . . . . . . . . . . 49

2.5 Cirurgias da aplicação de Gauss . . . . . . . . . . . . . . . . . . . 51

2.5.1 Cirurgia $S^{-} \ldots \ldots \ldots \ldots \ldots$. . . . . . . . . . . 51

2.5.2 Cirurgia $S^{-+} \ldots \ldots \ldots \ldots \ldots$. . . . . . . . . . . . . 52

2.5.3 Cirurgia $S^{+} \ldots \ldots \ldots \ldots \ldots$. . . . . . . . . . . . . 52

3 Invariantes de aplicações estáveis $\quad 55$

3.1 Grafos associados às superfícies com um conjunto de curvas prefixado . . . 55

3.2 Grafos como invariantes de aplicações estáveis . . . . . . . . . . . . . 58

3.3 Invariantes locais de aplicações estáveis . . . . . . . . . . . . . . . . . . 59

3.3.1 Relação entre cúspides e característica de Euler . . . . . . . . . . . 61

3.4 Outras dissertações sobre resultados relacionados ao que estudamos . . . . 70

4 Invariantes de aplicações de Gauss estáveis $\quad 73$

4.1 Grafos de aplicações de Gauss estáveis . . . . . . . . . . . . . . . . . 74

4.2 Transições da aplicação de Gauss . . . . . . . . . . . . . . . . . . 75

4.2.1 Grafo da transição lábios . . . . . . . . . . . . . 76

4.2.2 Grafo da transição bicos . . . . . . . . . . . . . . . . 76

4.2.3 Exemplos: realização de grafos . . . . . . . . . . . . . . . 77

4.3 Efeitos das cirurgias nos grafos das aplicações de Gauss de superfícies . . . 80

4.3.1 Grafo da cirurgia $S^{-} \ldots \ldots \ldots$. . . . . . . . . 80

4.3.2 Grafo da cirurgia $S^{-+} \ldots \ldots \ldots \ldots$. . . . . . . . . . 82

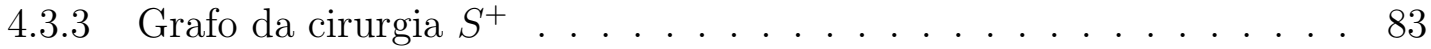


4.4 Realização de grafos bipartidos . . . . . . . . . . . . . . . . . 84

4.4.1 Grafos bipartidos com número de arestas $\leq 3 \ldots$. . . . . . . . 84

4.4.2 Realização de grafos árvore e grafos bipartidos . . . . . . . . . . . 86

4.4.3 Cúspides da aplicação de Gauss estável . . . . . . . . . . . . . . . 88

4.5 Algoritmo para a realização dos grafos . . . . . . . . . . . . . . . 95

4.6 Matrizes de aplicação de Gauss estáveis . . . . . . . . . . . . . . . . . 96 


\section{Resumo}

Oliveira, F. H.. Grafos e aplicação de Gauss estáveis. 2016. 111 f. Dissertação (Mestrado em Matemática) - Instituto de Ciências Matemáticas e de Computação (ICMC/USP), São Carlos - SP.

O objetivo desta dissertação é estudar grafos com pesos nos vértices como um invariante global das aplicações de Gauss estáveis de superfícies compactas e orientadas. Apresentaremos também alguns invariantes locais que são importantes no estudo de aplicações estáveis. Abordaremos o problema de realização de grafos por aplicações de Gauss estáveis, considerando também um destes invariantes, o número de cúspides destas aplicações. Finalmente, usaremos matrizes para representar estes invariantes e definiremos classes de equivalências para estes representantes. Esta foi uma ideia que surgiu no final deste trabalho de mestrado.

Palavras-chave: Grafos, Superfícies, aplicações de Gauss estáveis. 


\section{Abstract}

Oliveira, F. H.. Grafos e aplicação de Gauss estáveis. 2016. 111 f. Dissertação (Mestrado em Matemática) - Instituto de Ciências Matemáticas e de Computação (ICMC/USP), São Carlos - SP.

The propose of this work is to study graphs with weights at the vertices as a global invariant of stable Gauss applications on compact and oriented surfaces. We also present some local invariants that are important to the study of stable applications. We approach the problem of realization of graphs by stable Gauss applications, also emphasizing one of these invariants, the number of cusps of these applications. Finally, we use matrices to represent these invariants and define equivalence classes for these representatives. This idea appeared at the end of this master's thesis.

Key-words: Graphs, Surfaces, stable Gauss maps. 


\section{Introdução}

Em 1955, Whitney publicou o artigo [53], que é base para uma teoria sobre aplicações estáveis do plano no plano. Neste artigo, Whitney classifica os germes de aplicações estáveis do plano no plano como sendo germes do tipo regular, germes do tipo dobra, e germes do tipo cúspide. No final da década de 50, Thom em [49], percebeu que esses resultados poderiam ser agregados a uma nova teoria: a teoria das singularidades. Em 1986, J. Montaldi em [36], apresentou um método para estudar singularidades através de teoria de contato entre subvariedades.

Com a teoria de singularidades foi possível reinterpretar resultados clássicos da geometria diferencial local das superfícies no espaço Euclidiano $\mathbb{R}^{3}$. Isso permitiu a descoberta de resultados fascinantes sobre esta geometria. Esses novos resultados seguiram da sugestão de René Thom que consiste no estudo do contato da superfície com conjuntos especiais como por exemplo planos, linhas, esferas, círculos, etc.

Nesse trabalho, estudamos o problema da realização de grafos de aplicações de Gauss estáveis de superfícies fechadas sem bordo, nos baseando principalmente no artigo [30] de Mendes, Moraes e Romero, publicado em 2011, onde os autores mostram que qualquer grafo bipartido com pesos pode ser realizado por uma tal aplicação. Para chegar nesta realização estudamos as transições dadas por Bruce, Giblin e Tari em [9], e as cirurgias $S^{-}, S^{-+}$e $S^{+}$definidas em [30] por Mendes, Moraes e Romero, pois algumas transições, a saber, $A_{3}^{+}$e $A_{3}^{-}$, e as cirurgias alteram o conjunto singular de maneira conveniente para a realização dos grafos.

O presente trabalho foi dividido da seguinte forma:

No Capítulo 1, veremos conceitos e resultados preliminares necessários para o desenvolvimento do trabalho. Começaremos com os complexos regulares e característica de Euler de uma superfície compacta, seguida de alguns conceitos relevantes da Teoria de 
Grafos. Apresentaremos também alguns conceitos da Teoria de Singularidades, do ponto de vista da Topologia Diferencial, tais como k-jatos, a topologia $C^{\infty}$ de Whitney, alguns conceitos de aplicações estáveis e a noção de conjuntos singulares, considerando em particular, o caso das aplicações de Gauss de superfícies. Por fim, apresentaremos o conceito de germes de aplicações diferenciáveis e desdobramento. As principais referências utilizadas são: $[15,21,23,26,29,31,32,46,52]$.

No Capítulo 2, apresentaremos os casos estáveis das singularidades da aplicação de Gauss, utilizando a classificação dada por Bruce, Giblin e Tari em [9], que é feita a partir do estudo das funções altura de uma superfície $M$ na direção normal à superfície e dos germes de $\mathbb{R}^{2} \rightarrow \mathbb{R}^{2}$. Além disso, apresentaremos as transições locais da aplicação de Gauss para famílias de superfícies a 1-parâmetro. Estas transições são vistas do ponto de vista local. Em seguida serão apresentadas as cirurgias da aplicação de Gauss, a saber, as cirurgias $S^{-}, S^{-+}$e $S^{+}$. Estas cirurgias foram introduzidas em [30]. Para gerar as figuras que ilustram a seção de transições locais utilizamos o software SuperfíciesII de Montesinos (ver [35]), que está disponível em sua página.

No Capítulo 3, estudaremos os grafos associados às superfícies com um conjunto de curvas prefixadas, apresentaremos os invariantes locais de aplicações estáveis, e estudaremos uma demonstração para um caso particular do Teorema de Quine [41].

No Capítulo 4, estudaremos os grafos associados às aplicações de Gauss estáveis, apresentando o efeito das transições locais $A_{3}$ e das cirurgias definidas no Capítulo 2 sobre estes grafos. Utilizaremos também as transições locais e cirurgias para tratar do problema da realização de grafos por aplicações de Gauss estáveis, fazendo o uso, sempre que possível do resultado de Quine [41] para análise das cúspides da aplicação de Gauss. A transição $A_{4}$ ajuda na realização do grafo com um número menor de cúspides e em [42] temos um teorema que analisa o número de cúspides após a realização de transições. Porém não abordaremos aqui as transições $A_{4}$.

Apresentaremos um algoritmo que pode ser utilizado para realizar a aplicação de Gauss de qualquer grafo bipartido com pesos.

Para finalizar, introduziremos classes de matrizes para representar os invariantes: grafos com pesos, número de cúspides e número de pontos duplos. 


\section{Capítulo 1}

\section{Resultados preliminares}

Neste capítulo, veremos alguns conceitos e resultados preliminares necessários para o estudo de grafos de superfícies fechadas e orientadas em $\mathbb{R}^{3}$ com aplicações de Gauss estáveis. Não provaremos estes resultados, pois no nosso estudo daremos mais ênfase na Teoria de grafos.

Começaremos com os complexos regulares para definir a característica de Euler de uma superfície compacta e orientável, seguida de alguns conceitos relevantes da Teoria de Grafos. Na segunda parte, apresentaremos conceitos da Teoria de Singularidades, do ponto de vista da Topologia Diferencial, tais como os k-jatos, a topologia $C^{\infty}$ de Whitney, aplicações estáveis e a noção de conjuntos singulares. Em seguida, definiremos a aplicação normal de Gauss para uma superfície. Finalizaremos com conceitos da Teoria de Singularidades, tais como os germes e a Teoria da catástrofe de Thom-Zeeman.

\subsection{Característica de Euler}

Nesta seção veremos os complexos, e definiremos complexo simplicial para introduzir um invariánte topológico completo. Veremos a característica de Euler para superfícies compactas e orientadas, com bordo ou sem bordo. A característica de Euler (ver [32]), de superfícies compactas com bordo ou superfícies compactas sem bordo, será últil na hora de associar os grafos como invariantes topológicos de superfícies. Ela serve também para diferenciar superfícies compactas. 
Temos que se $\alpha$ é um invariante topológico e X e Y são topologicamente equivalentes, então $\alpha(\mathrm{X})=\alpha(\mathrm{Y})$. Logo a importância dos invariantes topológicos é que quando eles são diferentes para dois espaços temos que esses espaços não são homeomorfos, ou seja, não são topologicamente equivalentes.

\subsubsection{Complexos}

Utilizamos [32] como referência para definir os complexos.

Definição 1.1.1. Uma n-célula é um conjunto cujo interior é homemorfo a um disco $n$-dimensional $D_{n}=\left\{x \in \mathbb{R}^{n}:\|x\|<1\right\}$ com a propriedade adicional de que sua fronteira deve ser dividida em um número finito de células com dimensões menores, chamadas faces da n-célula. Seja $\tau$ uma n-célula, escrevemos que $\sigma<\tau$ se $\sigma$ é uma face de $\tau$.

Observação 1.1.2. 1. Uma 0-célula é um ponto $A$.

2. Uma 1-célula é um segmento de reta $a=A B$, e $A<a, B<a$.

3. Uma 2-célula é um polígono (por exemplo, um triângulo), assim temos $\sigma=\Delta A B C$, e temos $A B, B C, A C<\sigma$. Observe que $A<A B<\sigma$, assim $A<\delta$.

Indutivamente, temos que as faces de uma n-célula são as células de dimensão menor: pontos finais de uma 1-célula são as 0-células, a fronteira de uma 2-célula consiste de 1-células e 0-células, e as faces de uma n-célula consiste de 0-células, 1-células,...,(n-1)células, ver Figura 1.1. Estas células juntas, formam os complexos.

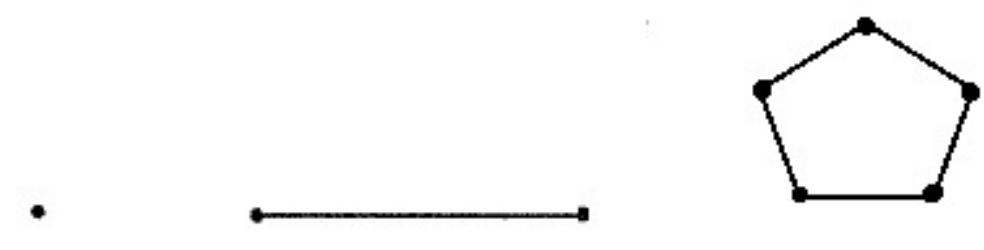

Figura 1.1: 0-,1-,2-células

O primeiro círculo da Figura 1.2 não é uma célula, pois temos que sua fronteira é a $S^{1}$ que não é homeomorfo a um disco $D_{1}$ (um segmento de reta), diferente do segundo círculo 
que tem uma fronteira de três partes da $S^{1}$ e três pontos, que são 1-células e 0-células respectivamente.

Exemplo 1.1.3. Uma célula 2-dimensional é um polígono, por exemplo um hexágono, com segmentos de reta (arestas do polígono) e pontos (vértices do polígono).

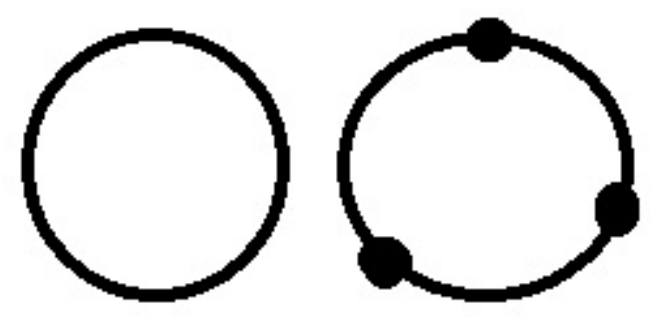

Figura 1.2: Exemplo de um círculo que não é uma célula, e de um círculo que é célula.

Exemplo 1.1.4. Uma célula 3-dimensional é um poliedro sólido, por exemplo, um tetraedro, com polígonos (face do poliedro), segmentos de reta (arestas do poliedro) e pontos (vértices do poliedro).

Células são coladas para formar os complexos, colando arestas com arestas, vértice com vértice e identificando células de dimensão maior de uma maneira similar (não queremos, por exemplo, colar uma face retangular com uma face triangular).

Definição 1.1.5. O $\boldsymbol{n}$-complexo $\boldsymbol{K}$, também denotado por $\boldsymbol{K}_{n}$ é a união de $k$-células com $0 \leq k \leq n$

$$
K=\cup_{k=0}^{n}(k \text {-células })
$$

tal que:

i) se $\sigma$ é uma célula de $\boldsymbol{K}$, então todas as faces de $\sigma$ são elementos de $\boldsymbol{K}$, ii)se $\sigma$ e $\tau$ são células de $\boldsymbol{K}$, então $\operatorname{Int}(\sigma) \cap \operatorname{Int}(\tau)=\varnothing$. 

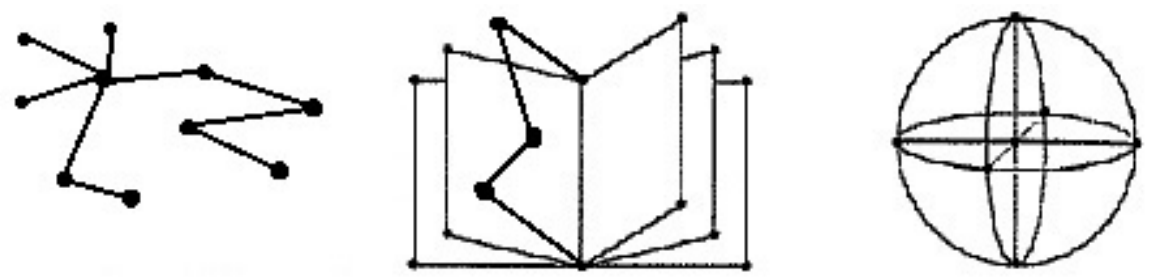

Figura 1.3: Exemplos de n-complexos

A dimensão n de K é a dimensão de sua célula de maior dimensão, ver Figuras 1.3 e 1.5.

A condição ii) nos diz que as situações da Figura 1.4 não podem ocorrer. Na Figura 1.5 sabemos que todos eles são topologicamente equivalentes, então eles representam o mesmo espaço topológico mas com diferentes estruturas complexas.
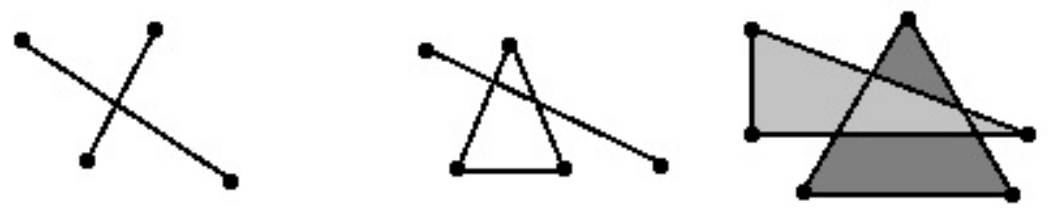

Figura 1.4: Interseção, do interior de células, não vazia
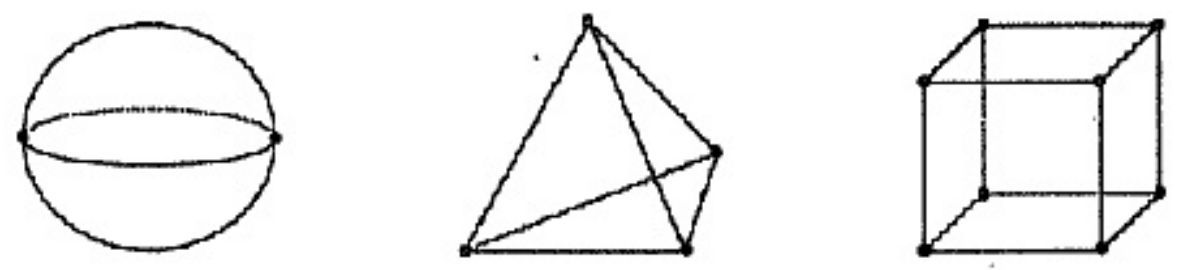

Figura 1.5: Diferentes complexos de uma esfera

Definição 1.1.6. Seja $K$ um n-complexo, o espaço subjacente $X$ de $K$ é o conjunto:

$$
\boldsymbol{X}=|\boldsymbol{K}|=\{x: x \in \sigma \in \boldsymbol{K}, \sigma \text { é uma célula em } \boldsymbol{K}\}
$$

O espaço subjacente é um conjunto de pontos, diferente do n-complexo regular que é um conjunto de células.

Definição 1.1.7. [32] A característica de Euler de um n-complexo $K$, denotada por $\chi(\boldsymbol{K})$, é a soma alternada do número das células do complexo $\boldsymbol{K}$, ou seja, 


$$
\chi(\boldsymbol{K})=\sum_{k=0}^{n}(-1)^{k} \#(k-c e ́ l u l a s)
$$

onde \#(k-células) denota o número de k-células do complexo $\boldsymbol{K}$.

Exemplo 1.1.8. Para $\boldsymbol{K}$ um 2-complexo, denotando $\boldsymbol{F}=\#$ (2-células), $\boldsymbol{A}=\#$ (1-células)

e $\boldsymbol{V}=\#$ (0-células), a característica de Euler de $\boldsymbol{K}$, é dada por:

$$
\chi(\boldsymbol{K})=\boldsymbol{V}-\boldsymbol{A}+\boldsymbol{F}
$$

Exemplo 1.1.9. Vamos calcular a característica de Euler dos diferentes complexos de uma esfera da Figura 1.5. Na primeira esfera da figura temos $\boldsymbol{F}=2, \boldsymbol{A}=2$ e $\boldsymbol{V}=2 . \mathrm{Na}$ segunda esfera da figura temos $\boldsymbol{F}=4, \boldsymbol{A}=6$ e $\boldsymbol{V}=4$. Na terceira esfera da figura temos $\boldsymbol{F}=6, \boldsymbol{A}=12$ e $\boldsymbol{V}=8$. Nas três figuras temos que a característica de Euler dos diferentes complexos de uma esfera é sempre 2.

\subsubsection{Superfícies e característica de Euler}

Nesta subseção, apresentaremos resultados importantes de superfícies, e a característica de Euler de uma superfície a partir do 2-complexo.

Definição 1.1.10. Uma variedade n-dimensional é um espaço topológico tal que todo ponto x possui uma vizinhança homeomorfa (ou diremos simplesmente, equivalente) a um disco aberto n-dimensional, centrado em $x$ e com raio $r, \boldsymbol{D}^{n}(x, r)=\left\{y \in \mathbb{R}^{n}:\|y-x\| \leq r\right\}$. Uma 2-variedade é chamada superfície.

Exemplo 1.1.11. A esfera e o toro são exemplos de superfícies. De fato, $\varphi(\alpha, \beta)=$ $\left(\operatorname{sen}\left(\alpha+r_{1}\right) \operatorname{sen}\left(\beta+r_{2}\right), \cos \left(\alpha+r_{1}\right) \operatorname{sen}\left(\beta+r_{2}\right), \cos \left(\beta+r_{2}\right)\right)$ é um homeomorfismo entre o disco e uma vizinhança de um ponto $p$ da esfera. $\psi(\alpha, \beta)=\left(\left(r+\operatorname{sen}\left(\alpha+r_{1}\right)\right) \operatorname{sen}(\beta+\right.$ $\left.\left.r_{2}\right),\left(r+\cos \left(\alpha+r_{1}\right)\right) \operatorname{sen}\left(\beta+r_{2}\right), \cos \left(\beta+r_{2}\right)\right)$ é um homeomorfismo entre o disco e uma vizinhança de um ponto $p$ do toro, onde $r_{1}$ e $r_{2} \in(-\pi, \pi)$.

Definição 1.1.12. Uma n-variedade com bordo é um espaço topológico tal que todo ponto tem uma vizinhança topologicamente equivalente ou a um disco n-dimensional ou a meio disco, $\boldsymbol{D}_{+}^{n}(x, r)=\left\{y=\left(y_{1}, \ldots, y_{n}\right) \in \mathbb{R}^{n}:\|y\| \leq r\right.$ e $\left.y_{n} \geq 0\right\}$.

Pontos do bordo de uma n-variedade são os pontos cuja vizinhança é equivalente ao meio disco. 
Como exemplo de superfície com bordo, temos o cilindro circular reto finito onde seu bordo consiste de dois círculos. O bordo da faixa de Möebius é um único círculo, enquanto a esfera e o k-toro não possuem bordo, ou seja, são superfícies sem bordo.

Definição 1.1.13. Um 2-complexo $\boldsymbol{K}$ é um complexo simplicial quando $\boldsymbol{K}$ possui apenas células triangulares que satisfazem a condição adicional de que dois triângulos são indentificados ao longo de uma aresta ou somente em um vértice ou são disjuntos.

Definição 1.1.14. Uma triangulação de uma superfície (sem bordo) é um 2-complexo simplicial tal que:

1. cada aresta é identificada com exatamente uma outra aresta;

2. um dado vértice pode pertencer a $n$ triângulos, denotados por $T_{1}, T_{2}, \ldots, T_{n}$, de modo que nesta sequência, dois a dois triângulos são adjacentes e possuem uma aresta em comum e $T_{n}$ identifica com $T_{1}$ ao longo de uma aresta.

Definição 1.1.15. Uma triangulação de uma superfície (com bordo) é um 2-complexo simplicial tal que:

1. cada aresta é identificada com pelo menos uma outra aresta;

2. um dado vértice pode pertencer a $n$ triângulos, denotados por $T_{1}, T_{2}, \ldots, T_{n}$, de modo que nesta sequência, dois a dois triângulos são adjacentes e possuem uma aresta em comum e $T_{n}$ identifica com $T_{1}$ ao longo de uma aresta, ou $T_{1}$ e $T_{n}$ possuem uma aresta que pertence ao bordo.

3. uma aresta que não pertence ao bordo não pode ter ambos os vértices pertencentes ao bordo.

Ver exemplos nas Figuras 1.6 e 1.7.

Definição 1.1.16. Sejam $M_{1}$ e $M_{2}$ duas superfícies disjuntas. Em cada uma delas, removendo um pequeno disco, obtemos as superfícies $M_{1}^{*}$ e $M_{2}^{*}$ com uma nova componente de bordo cada, denotadas por $c_{1}$ e $c_{2}$. Colando $c_{1}$ e $c_{2}$ como exemplifica a Figura 1.9, formamos uma nova superfície, e essa superfície é denotada por $M_{1} \# M_{2}$, que é a soma conexa de $M_{1}$ e $M_{2}$. 

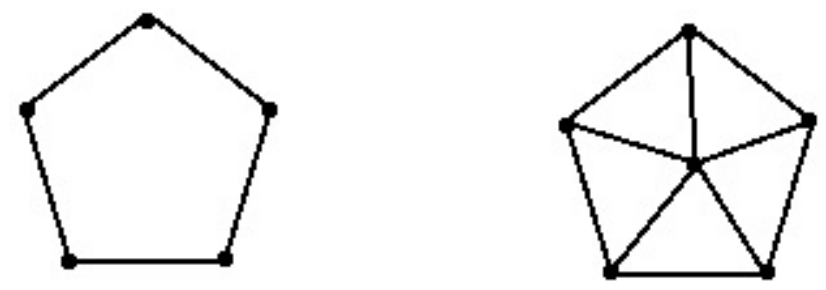

Figura 1.6: Triangulação de um polígono
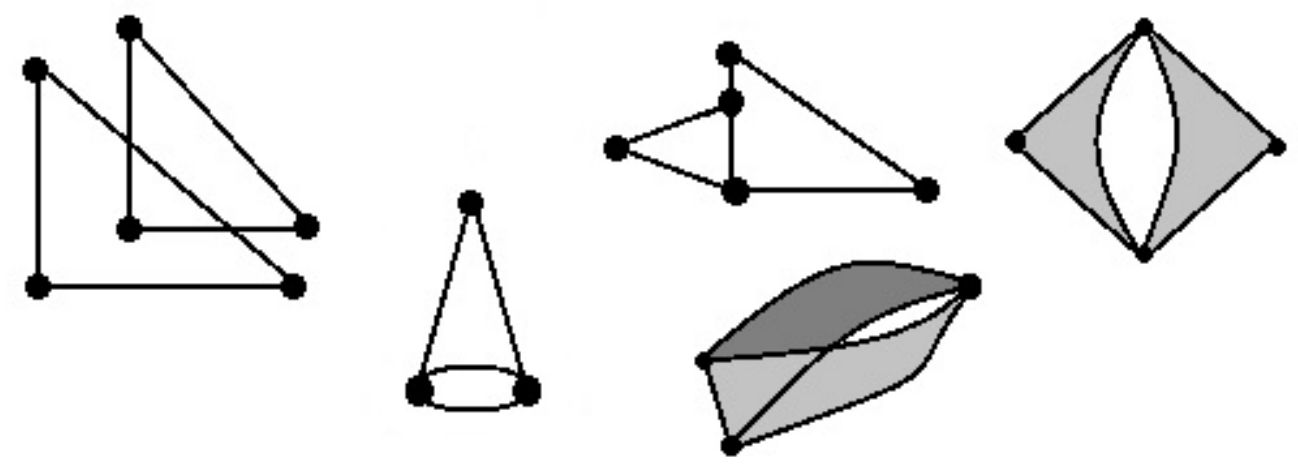

Figura 1.7: Não é triangulação

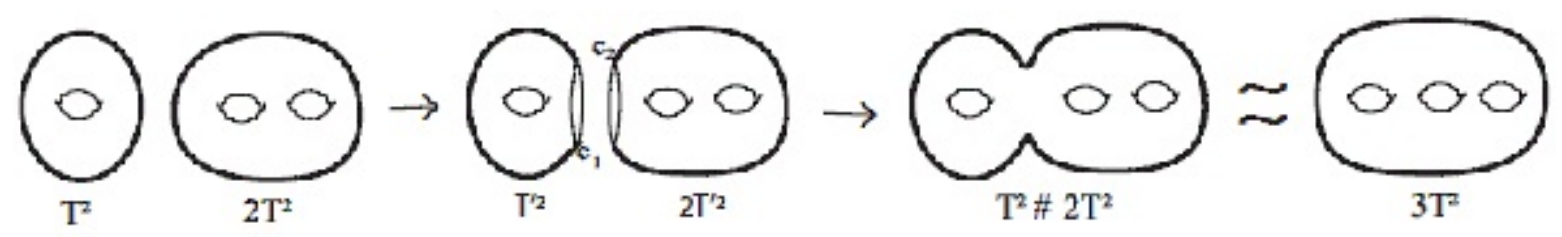

Figura 1.8: Soma conexa do toro com o 2-toro

A seguir, apresentaremos resultados sobre a classificação das superfícies compactas e conexas, que serão necessários para o Capítulo 4.

Teorema 1.1.17 ([32], Teorema 4.14, página 79). (Teorema de Classificação para superfícies) Toda superfície compacta, conexa e sem bordo é homeomorfa à esfera, ou à soma conexa de $n$ toros, ou à soma conexa de $n$ planos projetivos.

O teorema anterior nos diz que uma superfície compacta, conexa, orientável e sem bordo é homeomorfa à esfera, ou à soma conexa de $n$ toros.

Teorema 1.1.18 ([32], página 87, Teorema 4.17). (Teorema de Classificação para superfícies) Toda superfície compacta, conexa e com bordo é homeomorfa à esfera, ou à 
soma conexa de $n$ toros, ou à soma conexa de $n$ planos projetivos, com um número finito de discos removidos.

O teorema anterior nos diz que uma superfície compacta, conexa, orientável e com bordo é homeomorfa à esfera, ou à soma conexa de $n$ toros, com um número finito de discos removidos.

Teorema 1.1.19 ([32], Teorema 4.12, página 77). Uma superfície é compacta se, e somente se, qualquer triangulação possui um número finito de triângulos.

Teorema 1.1.20 ([32], Teorema 4.13, página 78). Uma superfície é conexa se, e somente se, uma triangulação pode ser arranjada na ordem $T_{1}, \ldots, T_{n}$, de modo que cada triângulo possui no mínimo uma aresta identificada com uma aresta de outro triângulo anterior.

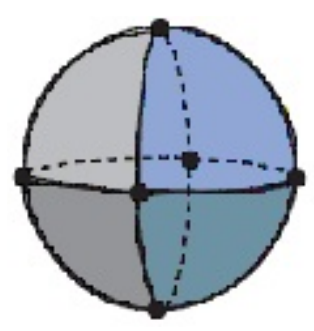

Figura 1.9: Triangulação da $S^{2}$

A Figura 1.8 ilustra uma triangulação da $S^{2}$, que podemos observar que satisfaz as condições para que o complexo simplicial seja uma triangulação.

A característica de Euler não nos diz exatamente com o que um espaço se parece, mas pode dar informações sobre similaridades e diferenças entre espaços, como acontece para grafos e árvores que veremos posteriormente.

Definição 1.1.21. A característica de Euler de uma superfície $M$ é definida como a característica de Euler de um 2-complexo $\boldsymbol{K}$ tal que $|\boldsymbol{K}|$ é homeomorfo a $M$.

Em [32], é provado que a característica de Euler de superfície está bem definida, provando que a definição independe do 2-complexo escolhido.

Na figura abaixo, temos um exemplo de triangulação do $D^{2}$, que possui a mesma característica de Euler de K. 

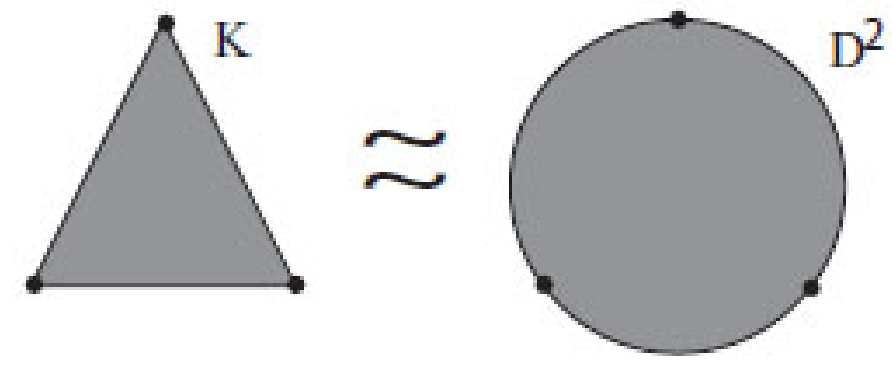

Figura 1.10: Característica de Euler do disco $D^{2}$

Teorema 1.1.22 ([32], Teorema 5.13, página 105). A característica de Euler é um invariante topológico completo para superfícies compactas, conexas.

Proposição 1.1.23 ([32], página 107). Se $M_{1}$ e $M_{2}$ são superfícies compactas e conexas, então

$$
\chi\left(M_{1} \# M_{2}\right)=\chi\left(M_{1}\right)+\chi\left(M_{2}\right)-2
$$

A esfera é o elemento neutro da operação soma conexa, \#, uma vez que ao realizar essa operação com outra superfície ela não se altera (a menos de homeomorfismo).

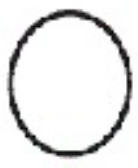

$5^{2}$

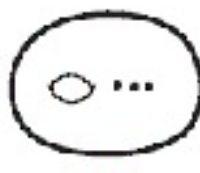

M

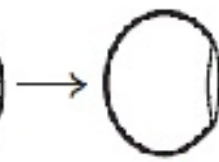

$5^{\prime}$

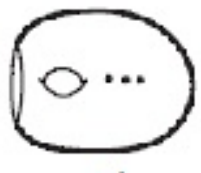

$\mathrm{M}^{\prime}$

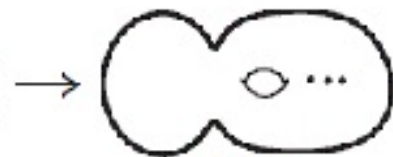

$\mathrm{S}^{2} \# \mathrm{M}$

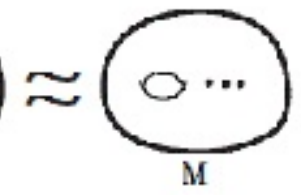

Figura 1.11: Soma conexa de uma superfície com $S^{2}$

O género de uma superfície compacta orientável M, é um invariante que corresponde ao número de toros que aparecem nos homeomorfismos dos Teoremas 1.1.17 e 1.1.18.

Definição 1.1.24. O género de uma superfície compacta orientável $M$, denotado por $g(M)$, é um invariante que corresponde ao número $k$, onde a superfície é homeomorfa ao k-toro(ver [32], página 10\%).

Do exemplo 1.1.9 temos $\chi\left(S^{2}\right)=2$. Vamos calcular a característica de Euler do toro. A triangulação tem 9 vértices, 27 arestas, 18 faces (Figura 1.12), logo a característica de 


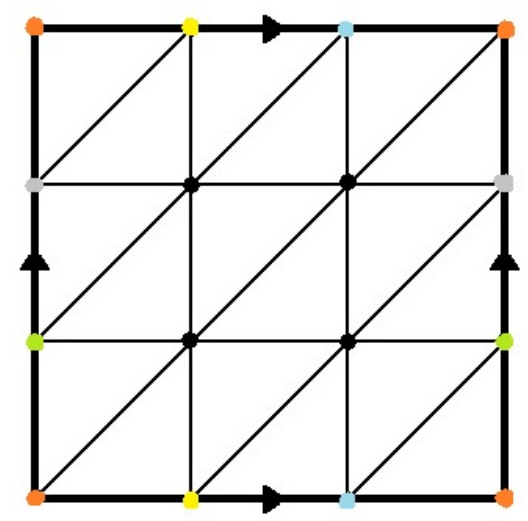

Figura 1.12: Triangulação do toro chato

Euler do toro é zero.

Da Proposição 1.1.23 temos o seguinte resultado.

Proposição 1.1.25. Se $M$ é uma superfície compacta orientável sem bordo, então

$$
\chi(\boldsymbol{M})=2-2 g(\boldsymbol{M})
$$

Se M é uma superfície orientável com k componentes de bordo, como cada componente de bordo é homeomorfo ao disco, adicionando um disco a cada componente de bordo obtemos uma superfície $\mathrm{M}^{\prime}$, tal que $\chi\left(\boldsymbol{M}^{\prime}\right)=\chi(\boldsymbol{M})+k$, e da Proposição 1.1.25 temos o seguinte resultado.

Proposição 1.1.26. Seja $\boldsymbol{M}$ uma superfície orientável com $k$ componentes de bordo. A característica de Euler de $\boldsymbol{M}$ é dada por

$$
\chi(\boldsymbol{M})=2-2 g(\boldsymbol{M})-k .
$$

\subsection{Grafos e Matrizes}

Nesta subseção, estudaremos conceitos básicos da Teoria dos Grafos necessários para o desenvolvimento deste trabalho. Utilizaremos [26], [31] e [32] como referência. Nesse trabalho consideraremos apenas os grafos conexos, e os chamaremos de grafos.

Definição 1.2.1. Um grafo $\mathcal{G}$ é um 1-complexo conexo. 
Uma aresta em $\mathcal{G}$ é uma 1-célula, um vértice é uma 0-célula. Denotaremos uma aresta que tenha como fronteira os vértices $u$ e $w$ pelo par $[u ; w]$ ou, simplesmente, por $u w$ quando não houver confusão. As arestas de um vértice $u$ são as 1-células tais que $u$ é uma face dessas 1-células. Quando $u$ possui uma única aresta, $u$ é chamado vértice extremo e neste caso, a aresta de $u$ é dita aresta extrema.

Observação 1.2.2. Como um grafo $\mathcal{G}$ é um 1-complexo, a característica de Euler é dada por

$$
\chi(\mathcal{G})=\boldsymbol{V}-\boldsymbol{A}
$$

onde $\boldsymbol{V}$ é o número de vértices e $\boldsymbol{A}$ é o número e arestas no grafo $\mathcal{G}$, já que grafos possuem somente vértices e arestas.

Um passeio é uma sequência de arestas do tipo $v_{0} v_{1}, v_{1} v_{2}, v_{2} v_{3}, \ldots v_{s-1} v_{s}$; s é o comprimento do passeio. Se todas as arestas do passeio são distintas, o passeio é chamado trilha; se $v_{0}=v_{s}$ o passeio é uma trilha fechada. Se, além das arestas, todos os vértices são distintos então temos um caminho e se $v_{0}=v_{s}$ temos um ciclo ( ver [31], página 28).

Os grafos podem ser representados por matrizes, uma maneira que facilita o estudo computacional dos grafos. A seguir definiremos a matriz de adjacência e a matriz de incidência. No final do Capítulo 4, apresentaremos algumas representações matriciais que mantêm informações da superfície e do grafo associado.

Sejam $\left\{a_{i}\right\}$ as arestas e $\left\{v_{j}\right\}$ os vértices do grafo $\mathcal{G}$.

Definição 1.2.3. [31] A matriz de adjacência de $\mathcal{G}$ é a matriz binária $\left(x_{i j}\right)$ onde $x_{i j}=1$ se existir uma aresta ligando o vértice $v_{i}$ ao vértice $v_{j}$, caso contrário $x_{i j}=0$. A matriz $\left(x_{i j}\right)$ é do tipo $n \times n$ onde $n$ é o número de vértices.

Definição 1.2.4. [31] A matriz de incidência de $\mathcal{G}$ é a matriz binária $\left(x_{i j}\right)$ onde $x_{i j}=1$ se a aresta $a_{j}$ tem como face o vértice $v_{i}$, caso contrário $x_{i j}=0$. A matriz matriz $\left(x_{i j}\right)$ é do tipo $n \times m$ onde $n$ é o número de vértices e $m$ é o número de arestas.

A seguir, veremos um exemplo de um grafo e suas matrizes de adjacência e de incidência, respectivamente. 


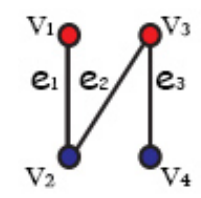

Figura 1.13: Exemplo de grafo

$$
\begin{gathered}
\left(\begin{array}{llll}
0 & 1 & 0 & 0 \\
1 & 0 & 1 & 0 \\
0 & 1 & 0 & 1 \\
0 & 0 & 1 & 0
\end{array}\right) \\
\left(\begin{array}{lll}
1 & 0 & 0 \\
1 & 1 & 0 \\
0 & 1 & 1 \\
0 & 0 & 1
\end{array}\right)
\end{gathered}
$$

\subsubsection{Ciclos independentes}

Quando associarmos grafos à aplicação de Gauss de superfícies, veremos uma relação entre o género da superfície e o número de ciclos independentes do grafo. Definiremos, a seguir, estes ciclos.

Seja $\mathcal{G}$ um grafo com q vértices e p arestas. Descreveremos um espaço vetorial associado ao grafo $\mathcal{G}$ : o "espaço cíclico". Por conveniência, esse espaço vetorial será considerado sobre o corpo finito de dois elementos $\mathbb{F}_{2}=(\{0,1\},+,$.$) .$

Definição 1.2.5. Seja $\mathcal{G}$ um grafo com vértices $v_{1}, v_{2}, \ldots, v_{q}$ e arestas $x_{1}, x_{2}, \ldots, x_{p}$. Definimos o espaço vetorial $\mathbb{V}$ como sendo o espaço vetorial com base $\left\{v_{1}, v_{2}, \ldots, v_{q}\right\}$ e coeficientes em $\mathbb{F}_{2}$, e o espaço vetorial $\mathbb{X}$ como sendo o espaço vetorial com base $\left\{x_{1}, x_{2}, \ldots, x_{q}\right\}$ e coeficientes em $\mathbb{F}_{2}$.

Definição 1.2.6. ([26], página 37) O operador bordo $\partial$ de $\mathbb{X}$ em $\mathbb{V}$ é tal que:

1. d é linear 
2. se $x=v_{i} v_{j}$, então $\partial x=v_{i}+v_{j}$.

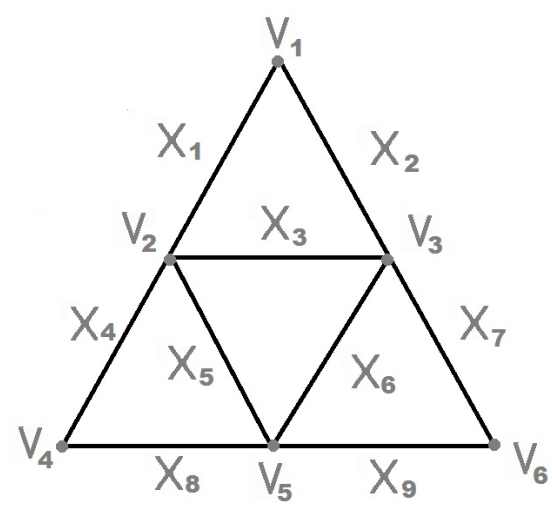

Figura 1.14: Um grafo para ilustrar o operador definido acima

Na Figura 1.14, considerando $x=x_{1}+x_{2}+x_{4}+x_{9}$ um vetor de $\mathbb{X}$ temos que:

$$
\begin{aligned}
\partial x=\left(v_{1}+v_{2}\right) & +\left(v_{1}+v_{3}\right)+\left(v_{2}+v_{4}\right)+\left(v_{5}+v_{6}\right) \\
= & v_{3}+v_{4}+v_{5}+v_{6},
\end{aligned}
$$

Teorema 1.2.7. O subconjunto $B=\left\{v_{1}+v_{2}, v_{1}+v_{3}, \ldots, v_{1}+v_{q}\right\}$ de $\mathbb{V}$ é uma base para a imagem do operador bordo.

Demonstração: Tomando $x_{i}=v_{j} v_{k}$, temos que $\partial x_{i}=v_{j}+v_{k}=\left(v_{1}+v_{j}\right)+\left(v_{1}+v_{k}\right)$, e portanto a imagem do operados bordo é gerada por elementos de B. Agora, seja $\left(v_{1}+v_{\omega}\right)$ em B então ele é imagem de um vetor de X que representa um caminho entre $v_{1} e v_{\omega}$.

Definição 1.2.8. Um vetor $x$ de $\mathbb{X}$ com bordo nulo, é um vetor cíclico de $\mathcal{G}$ e pode ser considerado como a união de ciclos disjuntos do grafo. A coleção de todos os vetores cíclicos formam um subespaço vetorial de $\mathbb{X}$, sobre $F_{2}$, chamado de espaço cíclico de $\mathcal{G}$.

Note que, por definição, o espaço cíclico de $\mathcal{G}$ é o núcleo do operador bordo, e pelo teorema do núcleo e da imagem temos que a dimensão do núcleo do operador bordo é p $-\mathrm{q}+1$.

Chamaremos a base do espaço cíclico de $\mathcal{G}$ de base cíclica, e chamaremos de ciclos independentes os vetores da base cíclica. 
Definição 1.2.9. A dimensão da base cíclica é chamada de número de Betti do grafo $\mathcal{G}$ e denotada por $\beta(\mathcal{G})$. Ou seja, $\beta(\mathcal{G})=p-q+1$

Definição 1.2.10. Uma árvore é um grafo $\mathcal{T}$ que não possui ciclos.

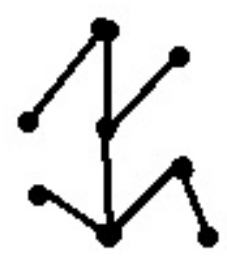

(a)

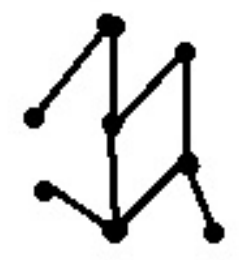

(b)

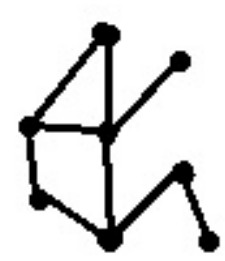

(c)

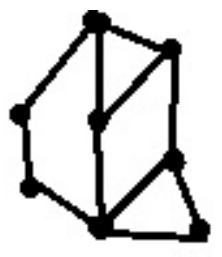

(d)

Figura 1.15: Exemplos de Grafos com e sem ciclos

Na Figura 1.15 temos quatro exemplos de grafos. Em (a) não ocorrem ciclos, em (b) e (c) ocorre um ciclo independente e em (d) ocorrem quatro ciclos independentes.

Definição 1.2.11. ([31] página 68) Uma árvore geradora de um grafo $\mathcal{G}$, com n vértices, é uma árvore $\mathcal{T}$ com $n-1$ arestas de $\mathcal{G}$.

A seguir, damos um exemplo de construção da base cíclica.

A partir de uma árvore geradora $\mathcal{T}$ do grafo $\mathcal{G}$ podemos construir uma base cíclica para o espaço cíclico de $\mathcal{G}$ da seguinte forma:

(i) Seja $\mathcal{T}^{0}=\left\{x_{i}\right\}$ o conjunto de todas as arestas de $\mathcal{G}$ que não estão em $\mathcal{T}$.

(ii) Adicionando a aresta $x_{i}$ à árvore $\mathcal{T}$ obtemos um grafo $\mathcal{G}_{i}$ que possui um ciclo $Z_{i}$.

(iii) $\left\{Z_{i}\right\}$ é uma coleção de ciclos independentes, pois cada ciclo tem uma aresta que os outros não possuem.

(iv) $\left\{Z_{i}\right\}$ é uma base cíclica pois possui elementos independentes e tem $\mathrm{p}-\mathrm{q}+1$ elementos que é a dimensão do espaço cíclico.

Observação 1.2.12. (a)Da Definição 1.2.9 e da Observação 1.2.2 segue que para todo grafo $\mathcal{G}$ temos que

$$
\chi(\mathcal{G})=1-\beta(\mathcal{G}) .
$$

(b)De (a) segue que se $\mathcal{G}$ é uma árvore então 

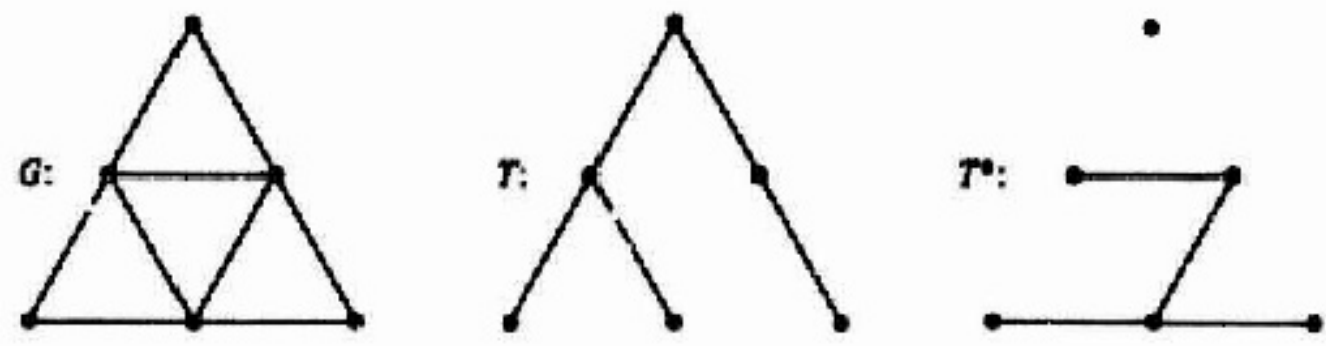

Figura 1.16: $\mathcal{G}, \mathcal{T}, \mathcal{T}^{0}$

$$
\chi(\mathcal{G})=1
$$

Teorema 1.2.13. [32] Característica de Euler é um invariante topológico para grafos.

\subsubsection{Grafos bipartidos}

Quando associarmos grafos às superfícies que possuem a aplicação de Gauss estável, veremos que esses grafos serão bipartidos. Veremos na Observação 1.3.28 que podemos separar as componentes conexas complementares do conjunto singular em dois conjuntos, tais que duas componentes conexas de um desses conjuntos não possuam uma fronteira em comum.

Definição 1.2.14. Um grafo é dito bipartido se é possível atribuir sinais + ou - a cada um de seus vértices de forma que cada aresta conecte apenas vértices de sinais opostos.

Teorema 1.2.15. ([31], página 29) Um grafo é bipartido se, e somente se, não contém ciclos de comprimento ímpar.

Corolário 1.2.16. Toda árvore é um grafo bipartido.

Exemplo 1.2.17. Na Figura 1.15 os exemplos (b) e (d) são grafos bipartidos pois todos os ciclos têm tamanho par, enquanto o exemplo (c) não é bipartido por ter ciclo de tamanho impar.

Definição 1.2.18. Um grafo com peso é um grafo em que a cada um dos seus vértices está associado um número natural. 
Definição 1.2.19. Dois grafos $\mathcal{G}_{1}$ e $\mathcal{G}_{2}$ são ditos isomorfos se existe uma bijeção $\rho: V\left(\mathcal{G}_{1}\right) \rightarrow V\left(\mathcal{G}_{2}\right)$ preservando adjacências; isto é, uw $\in A\left(\mathcal{G}_{1}\right)$, se, e somente se, $\rho(u) \rho(w) \in A\left(\mathcal{G}_{2}\right)$, onde $V\left(\mathcal{G}_{i}\right)$ é o conjunto dos vértices de $\mathcal{G}_{i}$ e $A\left(\mathcal{G}_{i}\right)$ é o conjunto das arestas de $\mathcal{G}_{i}, i=1,2$

Neste trabalho, consideraremos os grafos com pesos. Para simplificar, a partir de agora chamaremos apenas de grafos e no caso de grafos bipartidos utilizaremos a convenção de vértices na cor vermelha para vértices positivos e vértices na cor azul para vértices negativos.

\subsection{Preliminares da teoria de Singularidades}

Nesta seção, apresentaremos alguns conceitos da Teoria de Singularidades adotando o ponto de vista da Topologia Diferencial. Definiremos jatos, a Topologia de Whitney, aplicações de Gauss estáveis e conjuntos singulares.

\subsubsection{Aplicações estáveis e aplicação de Gauss}

As principais referências aqui são [15], [21], [53] e [55].

Definição 1.3.1. Seja $X$ uma variedade n-dimensional e seja $U=\left\{U_{i}\right\}$ uma cobertura de $X$ por abertos $\left\{U_{i}\right\}$ de $X$ com homemorfismos $\Phi_{i}: U_{i} \longrightarrow V_{i} \subset \mathbb{R}^{n}$ sobre abertos $V_{i} \subset \mathbb{R}^{n}$. O conjunto $\Phi=\left\{\left(\Phi_{i}, U_{i}\right)\right\}$ é dito um atlas e seus elementos são ditos cartas locais. Duas cartas $\left(\Phi_{i}, U_{i}\right)$ e $\left(\Phi_{j}, U_{j}\right)$ são $C^{r}$-compatíveis se:

$$
\Phi_{j} \circ \Phi_{i}^{-1}: \Phi_{i}\left(U_{i} \bigcap U_{j}\right) \longrightarrow \Phi_{j}\left(U_{i} \cap U_{j}\right) \text { é difeomorfismo } C^{r}
$$

No caso $r=0$, pedimos que seja um homemorfismo, e no caso $r=\infty$, que seja um difemorfismo $C^{\infty}$.

Definição 1.3.2. Um atlas em $X$ é $C^{r}$ se todo par de cartas são $C^{r}$-compatíveis. Dado um atlas $\phi C^{r}$ existe um único atlas $\Psi C^{r}$-maximal o qual contém todas as cartas $C^{r}$ compatíveis com $\Phi$. 
Definição 1.3.3. Um atlas maximal $\alpha C^{r}$ é dito uma $C^{r}$-estrutura diferenciável de $X$. Nesse caso $X$ é uma $C^{r}$-variedade diferenciável.

No caso $r=0$, dizemos que é uma variedade topológica, e no caso $r=\infty$, dizemos que $X$ é uma variedade diferenciável ou $C^{\infty}$.

Definição 1.3.4. Sejam $X, Y C^{r}$-variedades e $f: X \longrightarrow Y$. Duas cartas $(\Phi, U)$ de $X$ e $(\Psi, V)$ de $Y$ são adaptadas a $f$ se $f(U) \subset V$. Neste caso, dizemos que a representação local de $f$ nestas cartas (ou em $x \in U$ ) é $\Psi \circ f \circ \Phi^{-1}: \Phi(U) \longrightarrow \Psi(V)$. Dizemos que $f$ é $C^{k}$ em $x$ se a representação for $C^{k}$ onde $k \leq r$. Dizemos que $f$ é $C^{k}$ se o é em todos os pontos de $X$. No caso $k=\infty$, dizemos que $f$ diferenciável ou $C^{\infty}$.

Dadas duas variedades diferenciáveis $\mathbf{X}$ e $\mathbf{Y}$, denotamos por:

i) $C^{r}(X, Y)$ : espaço de todas as aplicações de $\mathrm{X}$ em $\mathrm{Y}$ de classe $C^{r}$. Se $\mathrm{X}=\mathrm{Y}$ escrevemos simplesmente $C^{r}(\mathrm{X})$.

ii) $C^{\infty}(X, Y)$ : espaço de todas as aplicações de $\mathrm{X}$ em $\mathrm{Y}$ de classe $C^{\infty}$. Se $\mathrm{X}=\mathrm{Y}$ escreveremos simplesmente $C^{\infty}(\mathrm{X})$.

Sejam $\mathbf{X}$ e $\mathbf{Y}$ variedades diferenciáveis e $f: \mathbf{X} \longrightarrow \mathbf{Y}$ uma aplicação diferenciável. Se $f$ tem posto máximo, então:

i) $f$ é uma imersão se, e somente se, $\operatorname{dim} \mathbf{X} \leq \operatorname{dim} \mathbf{Y}$;

ii) $f$ é uma submersão se, e somente se, $\operatorname{dim} \mathbf{X} \geq \operatorname{dim} \mathbf{Y}$;

iii) $f$ é um mergulho se, e somente se, $f$ é uma imersão injetiva.

Em [15] temos o seguinte resultado: uma superfície parametrizada M pode ser definida localmente como um subconjunto de $\mathbb{R}^{3}$, ou seja, na vizinhança de cada um de seus pontos, tem como parametrização uma imersão $f: U \subset \mathbb{R}^{2} \longrightarrow \mathbb{R}^{3}$.

Seja $M$ com parametrização $f: U \subset \mathbb{R}^{2} \longrightarrow M$ na vizinhança de um ponto $p \in M$ definimos uma aplicação $\mathcal{N}: M \longrightarrow S^{2}$ associada à $\mathrm{M}$ e à parametrização $f$ dada por

$$
\mathcal{N}=\frac{f_{u} \wedge f_{v}}{\left\|f_{u} \wedge f_{v}\right\|}
$$

onde $f(\mathrm{p})=\mathrm{q} \in f(\mathrm{U})$.

Definição 1.3.5. Uma superfície diferenciável $M$ é dita orientável se ela admite um 
campo diferenciável de vetores normais unitários definido em toda a superfície; a escolha de tal campo é chamada orientação de $M$.

Definição 1.3.6. Seja $M$ uma superfície parametrizada com uma orientação $\mathcal{N} . \quad A$ aplicação $\mathcal{N}$ é chamada aplicação de Gauss de $M$ e toma seus valores na esfera unitária $S^{2}$.

Em [15] temos um resultado que diz que a aplicação de Gauss independe da parametrização $f$ de $M$.

Definição 1.3.7. O conjunto singular de $\mathcal{N}, \Sigma \mathcal{N}$, é chamado de conjunto parabólico.

\subsubsection{Jatos}

A referência para esta subseção é o livro [21]. Usaremos os jatos para definir a topologia $C^{\infty}$ de Whitney.

Definição 1.3.8. Dadas $X$ e $Y$ variedades diferenciáveis e $x \in X$, dizemos que duas aplicações suaves $f, g: X \longrightarrow Y, \operatorname{com} f(x)=g(x)=y$, têm:

i) Contato de primeira ordem em $x$ se, e somente se, $(d f)_{x}=(d g)_{x}$, como aplicações de $T_{x} X \longrightarrow T_{y} Y$. Notação: $f \sim_{x} g$;

ii) Contato de ordem $k$, com $k \geq 2$, em $x$ se, e somente se, (df) : TX $\longrightarrow$ TY tem contato de ordem $k-1 \mathrm{com}(\mathrm{dg})$ em todo ponto de $T_{x} X$. Notação: $f \sim_{k} g ;$

Proposição 1.3.9. De modo equivalente, sejam $U$ um subconjunto aberto de $\mathbb{R}^{m}$ e $x$ um ponto de $U$. Sejam $f, g: U \longrightarrow \mathbb{R}^{m}$ aplicações diferenciáveis, então $f \sim_{k} g$ em $x$ se, e somente se,

$$
\frac{\partial^{|\alpha|} f_{i}}{\partial x^{\alpha}}(x)=\frac{\partial^{|\alpha|} g_{i}}{\partial x^{\alpha}}(x)
$$

para $\alpha$ multi-índice tal que $|\alpha| \leq k$, onde $f_{i}$ e $g_{i}$ são as funções coordenadas de $f$ e $g$ $e|\alpha|=\Sigma \alpha_{i}, \alpha_{i}$ as coordenadas do multi- indice $\alpha$ e com $1 \leq i \leq n$ e $x_{1}, \ldots, x_{m}$ as coordenadas de $x$ em $U$. Isto quer dizer que $f \sim_{k} g$ se, e somente se, $f$ e g possuem o mesmo desenvolvimento de Taylor de ordem $k$. 
A definição acima nos dá uma relação de equivalência, a relação "contato de ordem k", sobre o conjunto. A relação "contato de ordem k" definida acima é uma relação de equivalência também sobre o conjunto $C_{x, y}^{r}(X, Y)$, que é um subconjunto de $C^{r}(X, Y)$ cuja imagem de x é y. Denotaremos por $J_{x, y}^{k, r}(\mathrm{X} ; \mathrm{Y})=C_{x, y}^{r} \sim_{k}$. Quando $\mathrm{r}=\infty$, escreveremos $J_{x, y}^{k}(X, Y)$.

Definição 1.3.10. Os elementos de $J_{x, y}^{k}(X, Y)$ são chamados k-jatos de aplicações de $X$ em $Y$.

Definição 1.3.11. Seja $\sigma=j^{k}(f(x)) \in J^{k}(X, Y)$ um k-jato, onde $\sigma$ é um representante da classe de equivalência de $f j^{k}(f(x))$. Chamaremos $x \in X$ de fonte de $\sigma$ e $y=f(x)$ a meta de $\sigma$. Consequentemente temos a aplicação fonte $\alpha: J^{k}(X, Y) \longrightarrow X e$ aplicação meta $\beta: J^{k}(X, Y) \longrightarrow Y$, dadas respectivamente por $\alpha(\sigma)=x$ e $\beta(\sigma)=$ $f(x)$.

Seja $A_{m}^{k}$ o espaço dos polinômios de grau menor ou igual a $\mathrm{k}$ em $\mathrm{m}$ variáveis com coeficientes em $\mathbb{R}$ e que se anulam na origem. Temos o seguinte isomorfismo $A_{m}^{k} \approx \mathbb{R}^{N}$, onde $\mathrm{N}=\mathrm{d}(\mathrm{m}, \mathrm{k})+\mathrm{d}(\mathrm{m}, \mathrm{k}-1)+\ldots+\mathrm{d}(\mathrm{m}, 1)$ e $\mathrm{d}(\mathrm{m}, \mathrm{k})=\frac{(m+k-1) !}{(m-1) ! k !}$. Assim podemos considerar $A_{m}^{k}$ como sendo uma variedade $C^{\infty}$. De forma análoga, $B_{m, n}^{k}=\bigoplus_{i=1}^{n} A_{m}^{k}$ é também uma variedade $C^{\infty}$, cuja dimensão é $\operatorname{dim} B_{m, n}^{k}=\mathrm{n} \cdot \operatorname{dim} A_{m}^{k}=\mathrm{n} \cdot \mathrm{N}$.

Teorema 1.3.12. Sejam $X$ e $Y$ variedades $C^{\infty}$ tais que $\operatorname{dim} X=m$ e $\operatorname{dim} Y=n$. Então $J^{k}(X, Y)$ é uma variedade $C^{\infty}$ de dimensão $\left(m+n+\operatorname{dim} B_{m, n}^{k}\right)$ e as aplicações

$$
\alpha: J^{k}(X, Y) \longrightarrow X e \beta: J^{k}(X, Y) \longrightarrow Y
$$

são submersões $C^{\infty}$.

\subsubsection{A topologia $C^{\infty}$ de Whitney}

Vamos agora definir uma topologia muito importante em $C^{\infty}(X, Y)$, proveniente de abertos de $J^{k}(X, Y)(\operatorname{ver}[21])$.

Tomemos $\mathrm{X}$ e $\mathrm{Y}$ variedades diferenciáveis. Seja $\mathrm{k}$ um inteiro não negativo e $\mathcal{U}$ um subconjunto de $J^{k}(X, Y)$. Denotamos por $\mathcal{M}^{k}(\mathcal{U})$ o seguinte subconjunto de $C^{\infty}(\mathrm{X}, \mathrm{Y})$ : 


$$
\mathcal{M}^{k}(\mathcal{U})=\left\{f \in C^{\infty}(X, Y) ; j^{k} f(\alpha(\mathcal{U})) \subset \mathcal{U}\right\}
$$

Segue da definição que $\mathcal{M}^{k}(\mathcal{U}) \cap \mathcal{M}^{k}(\mathcal{V})=\mathcal{M}^{k}(\mathcal{U} \cap \mathcal{V})$.

Lema 1.3.13. [21] A família de subconjuntos $\left\{\mathcal{M}^{k}(\mathcal{U})\right\}$, onde $\mathcal{U}$ é um aberto de $J^{k}(X, Y)$ forma uma base para uma topologia em $C^{\infty}(X, Y)$.

Definição 1.3.14. A topologia induzida no Lema1.3.13 é chamada topologia $C^{k}$ de Whitney em $C^{\infty}(X, Y)$, denotada por $W_{k}$.

Definição 1.3.15. A topologia $C^{\infty}$ de Whitney em $C^{\infty}(X, Y)$ é aquela cuja base é dada $\operatorname{por} \mathcal{W}=\cup_{k=0}^{\infty} \mathcal{W}_{k}$

A definição dada acima utiliza a noção de k-jatos e está contextualizada com a seção anterior e com o que veremos a seguir.

Sejam $\mathcal{D}(\mathrm{X})$ e $\mathcal{D}(\mathrm{Y})$ os grupos de difeomorfismos suaves sobre a variedade $\mathrm{X}$ e $\mathrm{Y}$, respectivamente. O grupo $\mathcal{A}=\mathcal{D}(X) \times \mathcal{D}(\mathrm{Y})$ é um grupo que atua sobre $C^{\infty}(\mathrm{X}, \mathrm{Y})$.

Definição 1.3.16. A $\mathcal{A}$-órbita de $f$ é o subconjunto $\left\{h \in C^{\infty}(X, Y) ; \exists(\Phi, \psi) \in \mathcal{A}\right.$ tal que $h=$ $\left.\psi \circ f \circ \Phi^{-1}\right\}$

Definição 1.3.17. Sejam $f, g \in C^{\infty}(X, Y)$, com $X$ e Y variedades diferenciáveis. Dizemos que $f$ é $\mathcal{A}$-equivalente a $g$ (ou seja, estão na mesma $\mathcal{A}$-órbita), e denotamos por $f \sim_{\mathcal{A}} g$, se existirem $(\Phi, \psi) \in \mathcal{A}$ tal que $g=\psi \circ f \circ \phi^{-1}$.

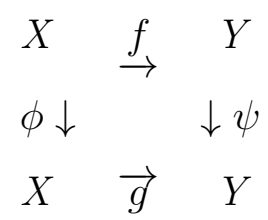

Definição 1.3.18. Dizemos que $f \in C^{\infty}(X, Y)$ é $\mathcal{A}$-estável se existe uma vizinhança $\mathcal{W}$ de $f$ na topologia $C^{\infty}$ de Whitney, tal que para toda aplicação $g \in \mathcal{W}$, vale a relação $f \sim_{\mathcal{A}}$ $g$.

Definição 1.3.19. Duas aplicações $f, g \in C^{\infty}(X, Y)$ são ditas isotopicamente estáveis se existe uma aplicação de classe $C^{\infty}, F: X \times[0,1] \rightarrow Y$ tal que

i) $F_{t}: X \rightarrow Y$ é estável para cada $t \in[0,1]$, sendo $F_{t}(p)=F(p, t)$

ii) $F_{0}=f$ e $F_{1}=g$. 
Note que duas aplicações isotopicamente estáveis são sempre $\mathcal{A}$-equivalentes.

Para facilitar, a partir de agora, vamos nos referir a aplicações $\mathcal{A}$-estáveis e $\mathcal{A}$-equivalentes como aplicações estáveis e aplicações equivalentes, respectivamente.

\subsubsection{Conjunto singular e contorno aparente}

Sejam M e N superfícies. Agora, apresentaremos resultados para aplicações estáveis $f$ : $\mathrm{M} \longrightarrow \mathrm{N}$, onde $\mathrm{M}$ é superfície compacta, orientada e sem bordo.

Os resultados e as definições desta subseção são devidos a Whitney e são baseados nas referências $[21,47,53,55]$.

Definição 1.3.20. Seja $f: M \longrightarrow N$ uma aplicação, o conjunto singular de $f$, denotado por $\Sigma f$, é o conjunto de pontos de $M$ nos quais o posto da diferencial df não é máximo. Um ponto $p \in M \backslash \Sigma f$ é dito ponto regular de $f$ e um ponto $y \in N$ é dito valor regular de $f$ se $f^{-1}(y)$ contém somente pontos regulares. O contorno aparente de $f, f(\Sigma f)$, é a imagem do conjunto singular de $f$.

Teorema 1.3.21. [47] O conjunto singular $\Sigma f$ de uma aplicação estável $f$ é uma subvariedade de codimensão $1 \mathrm{em} M$.

Proposição 1.3.22. Seja $p \in \Sigma f$ um ponto singular de uma aplicação estável $f$, então uma das seguintes situações ocorrem:

(a) $T_{p} \Sigma f \bigoplus \operatorname{ker}(d f)_{p}=T_{p} M$;

(b) $T_{p} \Sigma f=k e r(d f)_{p}$.

Se p é um ponto singular satisfazendo (a), então p é chamado de ponto de dobra. Por outro lado, se (b) ocorre, dizemos que p é um ponto de cúspide.

Proposição 1.3.23. [47] Se $f: M \longrightarrow N$ é uma aplicação estável, então para cada ponto $x \in M$, existem coordenadas locais $\left(x_{1}, x_{2}\right)$ centradas em $x$ e $\left(y_{1}, y_{2}\right)$ centradas em $f(x)$, tais que $f$ pode ser vista como uma aplicação de um aberto do plano no plano. Assim, $f$ é $\mathcal{A}$ - equivalente a uma das formas locais: 
1. $\left(x_{1}, x_{2}\right) \mapsto\left(y_{1}, y_{2}\right)=\left(x_{1}, x_{2}\right)$, neste caso $x$ é chamado ponto regular;

2. $\left(x_{1}, x_{2}\right) \mapsto\left(y_{1}, y_{2}\right)=\left(x_{1}^{2}, x_{2}\right)$, neste caso $x$ é chamado ponto de dobra;

3. $\left(x_{1}, x_{2}\right) \mapsto\left(y_{1}, y_{2}\right)=\left(x_{1} x_{2}-x_{1}^{3}, x_{2}\right)$, neste caso $x$ é chamado ponto de cúspide, ou seja, o conjunto singular de $f$ é formado por curvas de dobra com a possibilidade de pontos de cúspide isolados em $\Sigma f$.

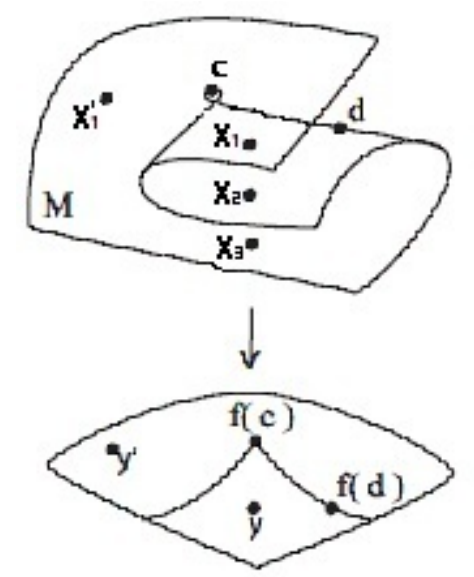

Figura 1.17: Pontos de dobra e cúspide

Na Figura 1.17 podemos ver que d é um ponto de dobra e c é uma cúspide. A cúspide c é o ponto de encontro de duas curvas singulares. Vemos também que as curvas singulares separam regiões de M onde a orientação é invertida, de regiões onde a orientação é preservada por $f$. Além disso, podemos ver que o ponto y possui três pré-imagens, os pontos $x_{1}, x_{2}$ e $x_{3}$, e o ponto $y^{\prime}$ possui apenas uma pré-imagem: $x^{\prime}{ }_{1}$.

A partir de uma aplicação $f$ podemos atribuir os sinais \pm a cada uma das componentes do complementar $M \backslash \Sigma f$.

Definição 1.3.24. Uma região conexa $R \in M \backslash \Sigma f$ é dita positiva se $f$ preserva a sua orientação, caso contrário, $R$ é dita negativa.

Definição 1.3.25. O fecho de cada componente conexa de $M \backslash \Sigma f$ cuja orientação é preservada por $f$ é denotado por $M_{i}^{+}$, enquanto o fecho de cada componente conexa de $M \backslash \Sigma f$ cuja orientação é invertida por $f$, é denotado por $M_{j}^{-}$. 
(a)

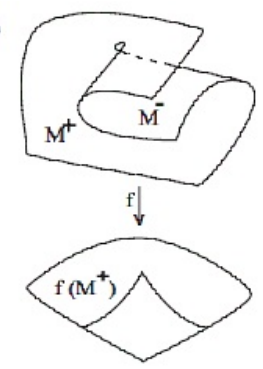

(b)

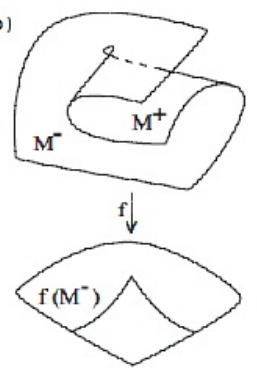

Figura 1.18: Sinal das regiões de uma superfície M

Podemos definir o sinal de qualquer cúspide de $f$, levando em consideração a orientação da região para a qual ela aponta, como mostra a Figura 1.18. Na Figura 1.18(a), a cúspide tem sinal positivo e na Figura 1.18(b) a cúspide tem sinal negativo.

Definição 1.3.26. O sinal de uma cúspide $C$ de uma aplicação estável $f$ é $s(C)=+1$ se $C$ aponta para uma região positiva de $f$ ou $s(C)=-1$, se $C$ aponta para a região negativa de $f$.
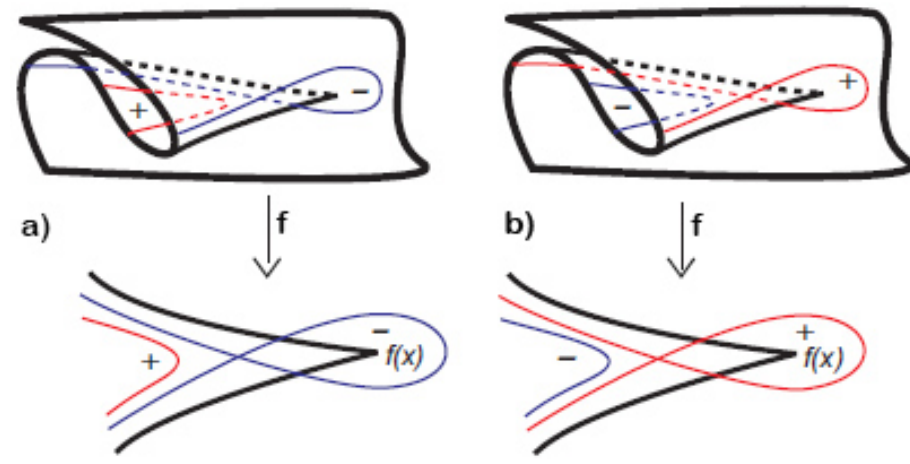

Figura 1.19: Sinal de cúspide

Lema 1.3.27. [21] Seja $f: M \longrightarrow N$ uma aplicação. O conjunto singular $\Sigma f$ de uma aplicação estável $f$ é formado por curvas fechadas, simples e disjuntas.

Observação 1.3.28. As componentes do complementar do conjunto singular de uma aplicação estável $f$ são levadas por $f$ em orientações opostas, logo $\Sigma f$ separa $M \backslash \Sigma f$ em componentes disjuntas. 
Proposição 1.3.29. [21] O conjunto singular de uma aplicação estável é composto por dobras e cúspides e além disso, os pontos de cúspides são isolados.

Segue então do Lema 1.3.27 que:

Proposição 1.3.30. [21] $O$ conjunto contorno aparente de $f$ estável , $f(\Sigma f)$, é uma coleção de curvas fechadas em $N$, cujas singularidades são pontos duplos transversais, ou seja, sem tangências e com possíveis pontos de cúspides isolados.

\subsubsection{Grau de aplicações}

Apresentaremos o grau de uma aplicação examinando a imagem inversa de um valor regular de uma aplicação própria $f: M \rightarrow N$, de classe $C^{1}$, onde $M$ e $N$ são variedades orientadas de mesma dimensão. Os resultados e definições abaixo podem ser encontrados em $[17]$.

Definição 1.3.31. Uma aplicação $f: M \rightarrow N$ é própria se a imagem inversa de um compacto é um compacto.

Proposição 1.3.32. As aplicações $f: M \rightarrow S^{2}$ contínuas, onde $M$ é compacto, são próprias.

Demonstração: De fato, dado $K \subset S^{2}$ compacto, $K$ é fechado, pois temos que $S^{2}$ é Haussdorf. Como $f$ é contínua, temos que $f^{-1}(K)$ é fechado em $M$, e como $M$ é compacto, segue que $f^{-1}(K)$ é compacto.

Sejam $M, N$ variedades orientadas, sem bordo, de mesma dimensão m, e $f: M \rightarrow N$ uma aplicação própria de classe $C^{1}$. De acordo com [17], para todo valor regular $y \in N$, de $f, f^{-1}(y)=\left\{x_{1}, \ldots, x_{n}\right\}$ é um conjunto finito.

Definição 1.3.33. Dizemos que um ponto $x_{k} \in f^{-1}(y)$ é positivo se $x_{k} \in M_{i}^{+}$ou negativo se $x_{k} \in M_{j}^{-}$, onde $M^{ \pm}$estão definidos em 1.3.25. 
Definição 1.3.34. Sejam $M, N$ variedades orientadas, sem bordo e de mesma dimensão $m$, e $f: M \rightarrow N$ uma aplicação própria de classe $C^{1}$. O grau de $f$ no ponto y, indicado por deg $f$ é a diferença entre o número de pontos positivos e o número de pontos negativos em $f^{-1}(y)$.

Teorema 1.3.35. [17] Seja $M$ uma variedade compacta, m-dimensional, orientada e sem bordo. A toda aplicação $f: M \rightarrow S^{m}$, de classe $C^{2}$, corresponde um inteiro $r$, tal que para todo valor regular $y$ de $f$, tem-se que $\operatorname{deg}_{y} f=r$.

Definição 1.3.36. O número $r=\operatorname{deg}_{y} f$ do Teorema 1.3.35 é o grau da aplicação $f$, ou simplesmente, deg $(f)$.

Proposição 1.3.37. 1. Se $f, g: M \rightarrow S^{m}$ são homotópicas de classe $C^{2}$, então $\operatorname{deg}(f)=\operatorname{deg}(g)$

2. Se $f: M \rightarrow S^{m}$ não é sobrejetiva, então deg $f=0$;

3. Se $f: S^{m} \rightarrow S^{m}$ é a aplicação identidade, entao deg $f=1$.

Corolário 1.3.38. Se o conjunto singular $\Sigma f$ de uma aplicação $f: S^{2} \rightarrow S^{2}$ é vazio, então deg $f=1$.

Demonstração: Se $\Sigma f=\emptyset$, então $f$ é homotópica a $I d: S^{2} \rightarrow S^{2}$, que por 3 da Proposição 1.3.37, possui grau um. Então por 1 da Proposição 1.3.37, segue que $\operatorname{deg} f=\operatorname{deg} I d=1$.

Observação 1.3.39. Desse corolário, temos que se $f: S^{2} \rightarrow S^{2}$ é tal que deg $f>1$, então o conjunto singular $\Sigma f \neq \emptyset$.

\subsubsection{Aplicação de Gauss e superfícies}

Nesta subseção, apresentaremos duas formas de definir a aplicação de Gauss. Uma delas é partir da curvatura principal. Com isso, veremos uma classificação da superfície (Definição 1.3.44). A outra, é ver localmente a superfície como o mergulho de um conjunto do $\mathbb{R}^{2}$ no $\mathbb{R}^{3}$, e dessa forma vamos considerar a aplicação de Gauss localmente como uma aplicação de $\mathbb{R}^{2}$ em $\mathbb{R}^{2}$. 
Definição 1.3.40. Seja $M$ superfície em $\mathbb{R}^{3}$. Podemos considerar seu plano tangente em $p$, denotado por $T_{p} M$, e uma direção $\Theta \in S_{p}^{1}$, onde $S_{p}^{1}$ é o círculo unitário em $T_{p} M$ com centro $p$. Assim, definimos o plano $\Pi_{p, \Theta}$ como sendo o plano passando por p que contém o vetor normal de $M, N_{p}$, e o vetor com ponto base em $p$ na direção $\Theta_{p}$, e chamamos a curva obtida pela interseção de $M$ e $\Pi_{p, \Theta}$, em uma vizinhança de $p$, de $\gamma_{\Theta}$.

Na Figura 1.20 temos um exemplo de curva $\gamma_{\Theta}$ (em branco), como definido acima, juntamente com os planos $T_{p} M$ e $\Pi_{p, \Theta}$.

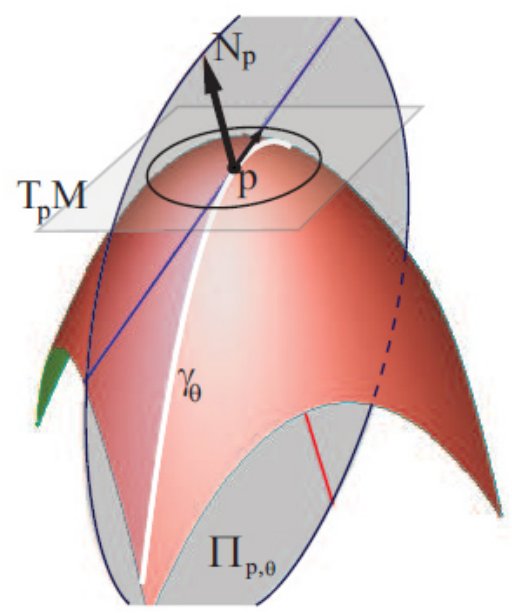

Figura 1.20: Curva $\gamma_{\Theta}$

Figura 1.20 retirada da dissertação [44].

Definição 1.3.41. Sejam $M$ uma superfície parametrizada em $\mathbb{R}^{3}$ e p um ponto de $M$. A função curvatura normal em p é a aplicação definida por

$$
\begin{aligned}
& k_{n}: S^{1} \longrightarrow \mathbb{R} \\
& \Theta \longmapsto k_{n}(\Theta)
\end{aligned}
$$

onde $k_{n}(\Theta)$ é a curvatura de $\gamma_{\Theta}$.

Temos que $k_{n}$ é uma aplicação contínua definida em $S_{p}^{1}$ que é um conjunto compacto, logo $k_{n}$ assume valor máximo e mínimo. Tomando $\Theta_{1}$ e $\Theta_{2}$ tais que $k_{1}=k_{n}\left(\Theta_{1}\right)$ e $k_{2}=$ $k_{n}\left(\Theta_{2}\right)$ sejam os valores máximo e mínimo, respectivamente, então definimos: 
Definição 1.3.42. $k_{1}$ e $k_{2}$, os valores máximo e mínimo de $k_{n}$ são chamados de curvaturas principais do ponto $p$.

Definição 1.3.43. Uma direção $\Theta$ cujo ponto tem $k_{1}$ ou $k_{2}$ como curvaturas principais, é chamada direção principal.

Definição 1.3.44. A curvatura Gaussiana de um ponto $p$ de uma superfície $M$ em $\mathbb{R}^{3}$ é o produto das curvaturas principais calculadas em p e a curvatura média é a média aritmética das curvaturas principais calculadas em p (ver [15],[38]).

Temos que a curvatura Gaussiana é uma função da superfície que associa a cada ponto p um valor $K(p)$ e a curvatura média é uma função da superfície que associa a cada ponto p um valor $\mathrm{H}(\mathrm{p})$, que denotamos simplesmente por $\mathrm{K}$ e $\mathrm{H}$ quando estivermos estudando elas em um ponto e não existir dúvida do ponto estudado.

Podemos classificar os pontos de uma superfície de acordo com o valor de suas curvaturas média e Gaussiana.

Definição 1.3.45. Um ponto $p$ de uma superfície $M$ em $\mathbb{R}^{3}$ é dito:

(i) Planar se $K(p)=0$ e $H(p)=0$.

(ii) Elíptico se $K(p)>0$ e $H(p) \neq 0$.

(iii) Hiperbólico se $K(p)<0$ e $H(p) \neq 0$.

(iv) Parabólico se $K(p)=0$ e $H(p) \neq 0$.

No caso da superfície ter uma aplicação de Gauss estável, não temos pontos planares, portanto podemos utilizar apenas a curvatura de Gaussiana, uma vez que a curvatura Gaussiana é suficiente para diferenciar as possibilidades restantes.

Em [4], são estudadas singularidades de aplicações de Gauss de superfícies parametrizadas por $X(x, y)=(x, y, f(x, y))$, onde a aplicação de Gauss é dada por $\mathcal{N}=$ $\left(-f_{x},-f_{y}, 1\right) /\left[1+\left(f_{x}\right)^{2}+\left(f_{y}\right)^{2}\right]^{1 / 2}, \mathcal{N}: M \rightarrow S^{2}$.

É possível estudar as singularidades da aplicação de Gauss de uma maneira mais fácil por uma projeção central a partir da origem para o plano $\mathrm{z}=1$, para se obter $\left(-f_{x},-f_{y}, 1\right)$. Então projetamos essa aplicação no plano xy e obtemos a aplicação $\mathcal{N}^{*}=\left(-f_{x},-f_{y}\right)$. 
Visto que a imagem da aplicação de Gauss $\mathcal{N}$ está contida no hemisfério superior de $S^{2}$, e a projeção central é um difeomorfismo do hemisfério superior no plano z = 1, a aplicação de Gauss modificada $\mathcal{N}^{*}$ terá as mesmas singularidades de $\mathcal{N}$. Logo podemos estudar as singularidades da aplicação de Gauss como singularidades de germes de aplicações do plano no plano. Sendo assim, $\mathcal{N}$ é singular precisamente nos pontos onde a matriz Jacobiana

$$
\left(\begin{array}{ll}
f_{x x} & f_{x y} \\
f_{x y} & f_{y y}
\end{array}\right)
$$

possui posto menor que dois, ou seja, quando o discriminante $\Delta=\left(f_{x y}\right)^{2}-f_{x x} f_{y y}$ é zero.

A partir de agora, diremos conjunto parabólico para nos referirmos ao conjunto singular de $\mathcal{N}$ e vamos nos referir a regiões de curvatura positiva $M_{i}^{+}$e curvatura negativa $M_{j}^{-}$ como regiões positivas e regiões negativas, respectivamente, mantendo também a notação $\mathcal{N}$ para uma aplicação de Gauss estável. $M_{i}^{+}$e $M_{j}^{-}$são regiões elípticas e hiperbólicas, respectivamente.

\subsection{Germes de aplicações diferenciáveis}

Todas as aplicações serão consideradas $C^{\infty}$. Estamos interessados no estudo local então definiremos a seguir os germes de aplicações. Para o nosso estudo são importantes as singularidades de funções, as singularidades das aplicações do $\mathbb{R}^{2}$ no $\mathbb{R}^{3}$, e as singularidades das aplicações do $\mathbb{R}^{4}$ no $\mathbb{R}^{3}$. Para esse último caso, temos as singularidades de Morin (Subseção 1.4.4) que serão usadas na classificação das singularidades da família de funções altura no Capítulo 2.

Sejam duas aplicações $f: U \longrightarrow \mathbb{R}^{p}$ e $g: V \longrightarrow \mathbb{R}^{p}$ definidas em vizinhanças $\mathrm{U}$ e $\mathrm{V}$ de um ponto $q \in \mathbb{R}^{n}$. Definimos a seguinte relação de equivalência:

$$
f \sim g \Leftrightarrow \exists \text { vizinhança } W \text { de } q, W \subset U \cap V \text { tal que }\left.f\right|_{w}=\left.g\right|_{w}
$$

Às classes de equivalência chamamos germes de aplicações no ponto q. Sem perda de generalidade tomamos $q=0 \in \mathbb{R}^{n}$. O germe de $f$ em 0 é denotado por

$$
f: \mathbb{R}^{n}, 0 \longrightarrow \mathbb{R}^{p}
$$


O conjunto dos germes $\mathbb{R}^{n}, 0 \longrightarrow \mathbb{R}^{p}$ é denotado por $O(n, p)$. Quando $\mathrm{p}=1$ (germes de funções), o conjunto é denotado simplesmente por $O_{n}$. O conjunto $m_{n}$ é o único ideal maximal de $O_{n}$, e $m_{n}=\left\{f \in O_{n} ; f(0)=0\right\}$.

\subsubsection{Desdobramento}

A perturbação (deformação) de uma singularidade pode gerar a mesma singularidade, nenhuma ou outras singularidades. Este é um estudo que pode ser feito pelos desdobramentos definidos a seguir. Um desdobramento a s-parâmetros de um germe $f_{0} \in m_{n} O(n, p)$ é um germe

$$
\begin{array}{lll}
F: \mathbb{R}^{n} \times \mathbb{R}^{s}, 0 & \longrightarrow \mathbb{R}^{p} \times \mathbb{R}^{s}, 0 \\
(x, u) & \longmapsto & (f(x, u), u)
\end{array}
$$

tal que $f(x, 0)=f_{0}(x)$. O germe $f(x, u)$ é chamado uma deformação de $f_{0}$.

1. O conjunto dos pontos críticos de F é

$$
\Sigma F=\left\{(x, u): D F_{x}(x, u) \text { é singular }\right\},
$$

2. O discriminante de $F, \Delta(F)$ é a imagem de $\Sigma F$, isto é, $\Delta(F)=F(\Sigma F)$.

3. O conjunto de bifurcação é definido por

$$
\operatorname{Bif}(F)=\left\{u \in \mathbb{R}^{s}: \exists x \in \mathbb{R}^{n}, 0 \text { com } f_{u} \text { instável em x }\right\} .
$$

Definição 1.4.1. Dois desdobramentos a s-parâmetros $F, G:\left(\mathbb{R}^{n} \times \mathbb{R}^{s}, 0\right) \rightarrow\left(\mathbb{R}^{p} \times \mathbb{R}^{s}, 0\right)$ de $f$ são isomorfos se existirem germes de difeomorfismos:

$$
\begin{aligned}
& \phi:\left(\mathbb{R}^{n} \times \mathbb{R}^{s}, 0\right) \rightarrow\left(\mathbb{R}^{n} \times \mathbb{R}^{s}, 0\right) \\
& \psi:\left(\mathbb{R}^{p} \times \mathbb{R}^{s}, 0\right) \rightarrow\left(\mathbb{R}^{p} \times \mathbb{R}^{s}, 0\right)
\end{aligned}
$$

que são desdobramentos a s-parâmetros das funçôes identidades sobre $\left(\mathbb{R}^{n}, 0\right)$ e $\left(\mathbb{R}^{p}, 0\right)$, respectivamente, tais que $G=\psi \circ F \circ \phi^{-1}$.

Definição 1.4.2. Dado um germe $h: \mathbb{R}^{t}, 0 \rightarrow \mathbb{R}^{s}, 0$ o pull-back de $F$ por $h$, denotado por $h^{*} F$, é o desdobramento a t-parâmetros

$$
h^{*} F(x, v)=(f(x, h(v)), v) .
$$


$F$ e $G$ são ditos equivalentes se existir um difeomorfismo $h: \mathbb{R}^{s}, 0 \rightarrow \mathbb{R}^{s}, 0$ tal que $G$ é isomorfo a $h^{*} F$. Esta é uma relação de equivalência. Se $G$ é um desdobramento a t-parâmetros de $f_{0}$ (t não necessariamente igual a s), dizemos que $G$ é induzido por $F$ se existir um germe $h: \mathbb{R}^{t}, o \rightarrow \mathbb{R}^{s}, 0$ tal que $G$ é isomorfo a $h^{*} F$.

Definição 1.4.3. $\quad$ a. F é versal se todos os desdobramentos de $f_{0}$ são induzidos por $F$.

b. F é trivial se é isomorfo ao desdobramento constante $(x, u) \mapsto\left(f_{0}(x), u\right)$.

c. $f_{0}$ é estável se todos os desdobramentos de $f_{0}$ são triviais.

Para mais informação ver, por exemplo, [45].

\subsubsection{Classificação dos germes de funções}

No próximo capítulo, veremos uma relação entre as singularidades da função altura e as singularidades da aplicação de Gauss. A tabela abaixo nos dá as singularidades de funções de $\mathbb{R}^{n}$ que será usada no próximo capítulo. Essa classificação é dada por Arnold e usada por diversos autores (para mais detalhes ver [45]).

Nome Forma Normal Corank

$\begin{array}{lrr}\text { Morse } & \sum_{i=1}^{n} \pm x_{i}^{2} & 0 \\ A_{k} & x_{n}^{k+1}+\sum_{i=1}^{n-1} \pm x_{i}^{2} & 1 \\ D_{k} & x_{n-1} x_{n}^{2} \pm x_{n-1}^{k-1}+\sum_{i=1}^{n-2} \pm x_{i}^{2} & 2\end{array}$

O desdobramento versal de uma singularidade $A_{k}$ é dado por

$$
\left(u_{1}, \ldots, u_{k-1}, \pm x_{n}^{k+1}+\sum_{i=1}^{k-1} u_{i} x_{n}^{i}+\sum_{i=1}^{n-1} \pm x_{i}^{2}\right)
$$

e o desdobramento versal de uma singularidade $D_{k}$ é dado por

$$
\left(u_{1}, \ldots, u_{k-1}, x_{n-1} x_{n}^{2} \pm x_{n-1}^{k-1}+\sum_{i=2}^{k-1} u_{i} x_{n-1}^{i-1}+u_{1} x_{n}+\sum_{i=1}^{n-2} \pm x_{i}^{2}\right)
$$

\subsubsection{Classificação dos germes $\mathbb{R}^{2} \rightarrow \mathbb{R}^{2}$}

As referências para a classificação dos germes $\mathbb{R}^{2} \rightarrow \mathbb{R}^{2}$ são [43] e [20]. Lembre que aplicação de Gauss pode ser vista como um germe de $\mathbb{R}^{2} \rightarrow \mathbb{R}^{2}, N^{*}=\left(-f_{x},-f_{y}\right)$, e então usaremos esta classificação. 
Seja $F: \mathbb{R}^{2}, 0 \rightarrow \mathbb{R}^{2}, 0$ um germe de uma aplicação suave. Se corank $(F) \leqslant 1$, então podemos mudar coordenadas e escrever $F$ na forma

$$
F(x, y)=(x, f(x, y))
$$

Denotamos por $a_{k, i}$ o coeficiente do monômio $x^{k-i} y^{i}$ na série de Taylor de $f$. Se $a_{1,1} \neq 0$ então $\mathrm{F}$ é um germe de um difeomorfismo, portanto $F \simeq_{\mathcal{A}}(x, y)$. Assim supondo $a_{1,1}=0$ temos as seguintes outras classes:

$\begin{array}{lrcr}\text { Nome } & \text { Forma Normal } & \text { Desdobramento Versal } & \mathcal{A}_{e}-\text { codim } \\ \text { Submersão } & (\mathrm{x}, \mathrm{y}) & (\mathrm{x}, \mathrm{y}) & 0 \\ \text { Dobra } & \left(\mathrm{x}, y^{2}\right) & \left(\mathrm{x}, y^{2}\right) & 0 \\ \text { Cúspide } & \left(x, x y+y^{3}\right) & \left(x, x y+y^{3}\right) & 0 \\ \text { Rabo de andorinha } & \left(x, x y+y^{4}\right) & \left(x, x y+y^{4}+u x^{2}, u\right) & 1 \\ \text { Lábios/bicos } & \left(x, y^{3}+x^{2} y\right) & \left(x, y^{3}+x^{2} y+u y, u\right) & 1 \\ \text { Borboleta } & \left(x, x y+y^{5}+y^{7}\right) & \left(x, x y+y^{5}+y^{7}+u y^{2}+v y^{3}, u, v\right) & 2 \\ \text { Ganso } & \left(x, y^{3}+x^{3} y\right) & \left(x, y^{3}+x^{3} y+u y+v x y, u, v\right) & 2 \\ \text { Gaivota } & \left(x, x y^{2}+y^{4}+y^{5}\right) & \left(x, x y^{2}+y^{4}+y^{5}+u y+v y^{3}, u, v\right) & 2\end{array}$

\subsubsection{Singularidades de Morin}

Nesta subseção, apresentaremos as singularidades de Morin que têm grande importância no estudo das singularidades estáveis das aplicações de Gauss de superfícies mergulhadas em $\mathbb{R}^{3}$ (ver [4]). Nesse trabalho, veremos duas formas de estudar as singularidades da aplicação de Gauss: na primeira estudaremos a aplicação de Gauss modificada $\mathcal{N}^{*}$, e na segunda estudaremos a aplicação catástrofe da família de funções alturas da superfície, e classificaremos as singularidades da família de funções alturas através das singularidades de Morin.

Definição 1.4.4. A forma normal com respeito a $\mathcal{A}$-equivalência de um germe com singularidade de Morin em $C^{\infty}(n, k+1)$ é:

$$
\left(y_{1}=x_{1}, \ldots, y_{k}=x_{k}, y_{k+1}=x_{n}^{r+1}+\Sigma_{i=1}^{r-1} x_{i} x_{n}^{i}+\sum_{j=k+1}^{n-1} \epsilon_{j} x_{j}^{2}\right)
$$


onde $r<k+1$ e $\epsilon_{j}= \pm 1$

Se $r=1$, o germe é chamado de germe do tipo dobra, se $r=2$, é chamado de germe do tipo cúspide, se $r=3$, é chamado de germe do tipo rabo de andorinha.

Usaremos as singularidades de Morin no Capítulo 2 para classificar as singularidades da família de função altura.

Observação 1.4.5. Uma singularidade de Morin é o desdobramento de uma singularidade de função do tipo $A_{k}$, para algum $k$.

A seguir, falaremos um pouco da teoria de catástrofe que mencionamos aqui.

\subsection{Teoria da catástrofe de Thom-Zeeman}

A referência para essa parte é o Capítulo 4 de [4]. A teoria da catástrofe de Thom-Zeeman é o estudo das singularidades de famílias de funções reais $F: Q \times N \rightarrow Q \times \mathbb{R}$.

Definição 1.5.1. O conjunto crítico (ou variedade catástrofe) da família $F$ é o conjunto

$$
C=\left\{(u, x) \in Q \times N ; \frac{\partial F}{\partial x}(u, x)=0\right\}
$$

Se $F$ é versal, então $C$ é uma variedade suave com a mesma dimensão de $Q$.

Definição 1.5.2. A aplicação catástrofe da família $F$ é a projeção $\chi: C \rightarrow Q$.

Uma família de funções reais $F: Q \times N \rightarrow Q \times \mathbb{R}$ é versal se, e somente se, $F$ é uma aplicação estável (ver [34]). Contudo, a aplicação catástrofe de uma família versal não é necessariamente estável.

Observação 1.5.3. Seja $\chi \in C^{\infty}(q, q)_{0}$ o germe no zero, de uma aplicação catástrofe de um desdobramento versal a q-parâmetros de um germe $f \in C^{\infty}(n, 1)$. Se $q=1$, $\chi$ ou é regular ou é uma singularidade de Morse. Se $q=2$, $\chi$ ou é regular, uma dobra, ou uma cúspide. Se $q=3$, $\chi$ ou é regular, uma dobra, uma cúspide, rabo de andorinha, ou um umbilico. Germes umbílicos em $C^{\infty}(3,3)_{0}$ não são estáveis.

No próximo capítulo, veremos na Seção 2.2 que a aplicação catástrofe da família de funções altura de uma superfície é a aplicação de Gauss dessa superfície. 


\section{Capítulo 2}

\section{Aplicações de Gauss, transições e cirurgias}

Neste trabalho, usaremos o termo cúspide de Gauss quando nos referirmos a uma cúspide da aplicação de Gauss.

Neste capítulo, vamos apresentar os casos das singularidades estáveis da aplicação de Gauss. Relacionaremos essas singularidades com as singularidades da função altura, utilizando a notação de Arnold $\left(A_{1}, A_{2}, A_{3}\right)$ apresentada em [3], e para isso analisaremos uma família de função altura, que pode ser vista como um desdobramento da função altura. Analisamos a aplicação catástrofe dessa família, uma vez que essa aplicação catástrofe é a aplicação de Gauss. Também apresentaremos exemplos de funções alturas que estão relacionadas com as singularidades de $\mathcal{A}_{e}-\operatorname{codim}=1$ da aplicação de Gauss, e veremos exemplos de família a 1-parâmetro de funções altura. As singularidades, neste caso, são do tipo (labios/bicos, ou rabo de andorinha) na aplicação de Gauss, e do tipo $\left(A_{3}, A_{4}\right)$ na função altura (ver [4] e [9]). Por último, vamos apresentar as cirurgias das aplicações de Gauss definidas em [30], chamadas de Cirurgia Neck, Cirurgia Neck-Beaks e Cirurgia Round e aqui chamaremos de $S^{-}, S^{-+} e S^{+}$, respectivamente. Estas cirurgias alteram o conjunto singular da aplicação de Gauss de maneira conveniente para o estudo que será apresentado no Capítulo 4. 


\subsection{Forma de Monge e familia de funções altura de uma superfície}

Seja M uma superfície suave em $\mathbb{R}^{3}$. O contato de $\mathrm{M}$ com planos em $\mathbb{R}^{3}$ é dado pela família de funções altura

$$
\begin{aligned}
\Pi: M \times S^{2} & \rightarrow \mathbb{R} \\
(p, u) & \mapsto<p, u>.
\end{aligned}
$$

Para cada u fixo, temos a função $\Pi_{u}$ que é a função altura na direção $\mathrm{u}$. Em $p \in M$ esta função descreve o contato de M com o plano pelo ponto p cujo vetor normal é u.

Podemos utilizar coordenadas locais convenientes em torno de $p_{0}$, tais que a superfície M é dada como o gráfico da função $z=f(x, y)$. Podemos escolher direções em $S^{2}$ em torno de $u=(0,0,1)$. Estas direções podem ser parametrizadas por $(\mathrm{a}, \mathrm{b}, 1)$. Então a família (alterada) de funções altura é dada, localmente, por

$$
\begin{aligned}
H: M \times \mathbb{R}^{2} & \rightarrow \mathbb{R} \\
((x, y),(a, b)) & \mapsto f(x, y)+a x+b y
\end{aligned}
$$

com $\Pi_{0}(x, y)=f(x, y)$. Queremos identificar geometricamente os tipos de singularidades da função $f$. Temos que $f$ é singular na origem se, e somente se, $f_{x}(0,0)=f_{y}(0,0)=0$, ou seja, se, e somente se, a direção $(0,0,1)$ é a direção normal a $\mathrm{M}$ em $p_{0}$.

A forma de Monge (local) para a vizinhança do ponto $p_{0}=(\mathrm{x}, \mathrm{y}, \mathrm{z})$ é dada por:

$$
\begin{aligned}
z & =f(x, y) \\
& =a_{0} x^{2}+a_{1} x y+a_{2} y^{2}+\text { termos de ordem mais alta. }
\end{aligned}
$$

Ou seja, a função altura $z$ da superfície na forma de Monge em (2.1) é a medida da "altura" da superfície até o plano tangente em $p_{0}$.

De [38] temos que toda superfície pode ser escrita localmente na forma de Monge, assim, o plano tangente na origem é o plano $x y$. Observe que sempre podemos considerar nosso estudo na origem, pois basta realizar uma translação da superfície em $\mathbb{R}^{3}$. 


\subsection{Aplicação Catástrofe da família de funções altura}

Nesta seção apresentaremos algumas caracterizações para as cúspides de Gauss, e o motivo de usar as funções alturas para estudar a aplicação de Gauss. Essa relação fica clara quando vemos que as aplicações catástrofes das famílias de funções alturas são as aplicações de Gauss. Ao caracterizar as cúspides de Gauss, no Teorema 2.2.3, percebemos que estes detalhes da superfície são perdidos ao usarmos um grafo para representá-la. No Capítulo 4 definiremos matrizes para representar as superfícies e seus conjuntos singulares, com o objetivo de preservar detalhes como estes que foram perdidos. Essa seção tem como referência o livro [4].

Seja $\Psi$ uma imersão de uma variedade n-dimensional em um espaço euclidiano $(n+1)$ dimensional. Para todo vetor unitário $v$ em $\mathbb{R}^{n+1}$ definimos $\Pi_{v}: M \rightarrow \mathbb{R}$ como:

$$
\Pi_{v}(p)=\Psi(p) \cdot v
$$

Todas as projeções juntas formam uma família parametrizada pela esfera unitária $S^{n}$ dada pela aplicação (família de funções altura)

$$
\Pi: S^{n} \times M^{n} \rightarrow S^{n} \times \mathbb{R}, \Pi(v, p)=\left(v, \Pi_{v}(p)\right)
$$

A função $\Pi_{v}$ pode ser vista localmente como um germe de função de $\mathbb{R}^{n}$, a aplicação $\Pi$ como um germe de aplicação de $\mathbb{R}^{2 n}$ em $\mathbb{R}^{n+1}$, a função $f: S^{2} \times M^{2} \rightarrow \mathbb{R}$ tal que $f(v, p)=\Pi_{v}(p)$ pode ser vista como um germe de função de $\mathbb{R}^{4}$.

O conjunto crítico $C$ da família $\Pi$ é o conjunto dos pares $(v, p), v \in S^{n}$ e $p \in M^{n}$, tais que $p$ é ponto crítico de $\Pi_{v}$, ou seja, $v$ é normal ao hiperplano tangente à imersão $\Psi$ em $p$. Assim, $C$ é identificado com os vetores normais unitários de $\Psi$. Se $M$ é orientável, então $C$ é duas cópias de $M$, onde cada uma corresponde a uma das orientações de $M$.

A aplicação catástrofe $\chi$ (Definição 1.5.2) da família de funções altura sobre uma hipersuperfície imersa é a aplicação de Gauss da hipersuperfície. De fato, seja $\chi: C \rightarrow$ $S^{n}$ a aplicação catástrofe da família $\Pi$, onde $(v, p) \in C$ se, e somente se, $v$ é normal a superfície em $p$, e $\chi(v, p)=v$. Logo $\chi$ é precisamente a aplicação de Gauss de $\Psi$.

O teorema a seguir nos mostra que existe um subconjunto aberto e denso no espaço de imersões onde o germe em $(v, p)$ da família $\Pi$ é um desdobramento versal do germe $\Pi_{v}$ em p para todo $(v, p) \in S^{2} \times M^{2}$. 
Teorema 2.2.1. [4] Seja $M^{2}$ uma superfície suave. Para um subconjunto denso aberto A do espaço de imersões $\left\{\Psi: M^{2} \rightarrow \mathbb{R}^{3}\right\}$, o germe em $(v, p)$ da familia $\Pi: S^{2} \times M^{2} \rightarrow S^{2} \times \mathbb{R}$ é um desdobramento versal do germe em $p$ de $\Pi_{v}$ para todo $(v, p) \in S^{2} \times M^{2}$.

A prova desse teorema se encontra no livro [4]. O teorema a seguir caracteriza as cúspides de Gauss.

O lema abaixo nos dá a relação entre as singularidades do germe de $\Pi$ e do germe de $\chi$.

Na definição da aplicação $\mathcal{N}^{*}$ temos que uma condição necessária para que a aplicação de Gauss seja estável é que a função altura tenha corank menor ou igual a um, ou seja, que a família de funções altura seja um desdobramento de uma singularidade $A_{k}$. Veremos que esses desdobramentos são singularidades de Morin (ver Definição 1.4.3 e Observação 1.4.4).

Lema 2.2.2. [4] Seja $\chi$ a aplicação catástrofe da família $\Pi: S^{2} \times M^{2} \rightarrow S^{2} \times \mathbb{R}$. O germe de $\chi$ em $(v, p)$ é uma cúspide (respectivamente, uma dobra ou germe regular) se, e somente se, o germe de $\Pi$ em $(v, p)$ é um rabo de andorinha (respectivamente, uma cúspide ou uma dobra).

Demonstração: O germe $\Pi$ é do tipo rabo de andorinha se $\Pi=\left(u, v, \pm x^{2} \pm\left(y^{4} \pm u y^{2} \pm v y\right)\right)$, do tipo cúspide se $\Pi=\left(u, v, \pm x^{2} \pm\left(y^{3} \pm v y\right)\right)$, ou do tipo dobra se $\Pi=\left(u, v, \pm x^{2} \pm y^{2}\right)$.

Para $\Pi=\left(u, v, \pm x^{2} \pm\left(y^{4} \pm u y^{2} \pm v y\right)\right)$ temos que o conjunto crítico $\mathrm{C}$ da família $\Pi$ é $C=\left\{(u, v, x, y) \in \mathbb{R}^{4} ; x=0\right.$ e $\left.v= \pm 2 u y \pm 3 y^{3}\right\}$, assim temos que $\chi$ é uma cúspide.

Para $\Pi=\left(u, v, \pm x^{2} \pm\left(y^{3} \pm v y\right)\right)$ temos que o conjunto crítico $\mathrm{C}$ da família $\Pi$ é $C=\left\{(u, v, x, y) \in \mathbb{R}^{4} ; x=0\right.$ e $\left.v= \pm y^{2}\right\}$, assim temos que $\chi$ é uma dobra.

Para $\Pi=\left(u, v, \pm x^{2} \pm y^{2}\right)$ temos que o conjunto crítico $\mathrm{C}$ da família $\Pi$ é $C=$ $\left\{(u, v, x, y) \in \mathbb{R}^{4} ; x=0\right.$ e $\left.y=0\right\}$, assim temos que $\chi$ é regular.

Logo temos que se o germe de $\Pi$ em (v,p) é um rabo de andorinha (respectivamente, uma cúspide ou uma dobra), então $\chi$ em (v,p) é uma cúspide (respectivamente, uma dobra ou germe regular).

Agora se $\chi$ é estável (regular, dobra ou cúspide), temos que $\Pi_{w}$ tem corank 0 ou 1 , onde $w$ é um vetor normal a $M^{2}$ em $p$. Para ver isso basta ver que $\chi$ tem as mesmas 
singularidades de $\mathcal{N}^{*}$, pois temos que a aplicação catástrofe da família de funções altura é a aplicação de Gauss, e a aplicação de Gauss tem as mesmas singularidades de $\mathcal{N}^{*}$, onde $\mathcal{N}^{*}=\left(\left(\Pi_{w}\right)_{x},\left(\Pi_{w}\right)_{y}\right) \cdot \mathcal{N}^{*}$ é estável quando a matriz abaixo tem rank 1 ou 2 , ou seja, $\Pi_{w}$ tem corank 0 ou 1.

$$
\left(\begin{array}{ll}
\left(\Pi_{w}\right)_{x x} & \left(\Pi_{w}\right)_{x y} \\
\left(\Pi_{w}\right)_{x y} & \left(\Pi_{w}\right)_{y y}
\end{array}\right)
$$

Se a aplicação $\chi$ é estável estamos no caso em que $\Pi_{w}$ tem corank 0 , temos que $\Pi_{w}$ é uma singularidade de Morse. Como $\Pi$ é um desdobramento versal de $\Pi_{w}$, segue que $\Pi$ é isomorfo a $\left(u, v, \pm x^{2} \pm y^{2}\right)$, que é a singularidade de Morin do tipo dobra.

Se a aplicação $\chi$ é do tipo dobra ou do tipo cúspide estamos no caso em que $\Pi_{w}$ tem corank 1 , e temos que $\Pi_{w}$ é uma singularidade $A_{k}$. O desdobramento versal de uma singularidade $A_{k}$ é um germe $G: \mathbb{R}^{k+1} \rightarrow \mathbb{R}^{k}$ tal que

$$
G\left(u_{1}, \ldots, u_{k-1}, x, y\right)=\left(u_{1}, \ldots, f\left(u_{1}, \ldots, u_{k-1}, x, y\right)\right)
$$

onde $f\left(u_{1}, \ldots u_{k-1}, x, y\right)= \pm y^{k+1}+\sum_{i=1}^{k-1} u_{i} x_{n}^{i}+x^{2}$. Logo como П é um desdobramento de $\Pi_{w}$ e $G$ é um desdobramento versal de $\Pi_{w}$, da Definição 1.4.3 segue que existe um germe $h: \mathbb{R}^{k-1} \rightarrow \mathbb{R}^{2}$ tal que $\Pi$ isomorfo a $h^{*} G, \operatorname{com} h^{*} G(u, v, x, y)=(u, v, f(h(u, v), x, y))$.

Temos que o conjunto singular de $h^{*} G$ é da forma $\left\{(u, v, x, y) \in \mathbb{R}^{4} ; \frac{\partial f}{\partial x}=x=0\right.$ e $\left.\frac{\partial f}{\partial y}=0\right\}$, onde podemos tirar $u$ em função de $v$ e $y$, ou $v$ em função de $u$ e $y$.

Por um lado temos que a aplicação $\chi$ é a projeção das duas primeiras coordenadas do conjunto singular de $h^{*} G$. Se a aplicação $\chi$ for uma cúspide temos que ter, por exemplo, $\frac{\partial f}{\partial y}=a u+b v y+c y^{3}$, para poder tirar $u$ em função de $v$ e $y$, isso ocorre apenas quando $h^{*} G$ é uma singularidade de Morin do tipo rabo de andorinha. Se $\chi$ for uma dobra temos que ter, por exemplo, $\frac{\partial f}{\partial y}=a u+b y^{2}$ para poder tirar $u$ em função de $y$, isso ocorre apenas quando $h^{*} G$ é uma singularidade de Morin do tipo cúspide.

Teorema 2.2.3. [4] Seja $U \subset \mathbb{R}^{2}$ um aberto, $e \Psi: U \rightarrow \mathbb{R}^{3}$ uma imersão suave. Se $p \in U$ é uma cúspide de Gauss de $\Psi$, então as declarações a seguir são verdadeiras. Por outro lado, se a aplicação de Gauss de $\Psi$ é estável, e satisfaz pelo menos uma das condições a seguir, então p é uma cúspide de Gauss. 
1. $\forall \epsilon>0 \exists Q_{1}, Q_{2}, Q_{3} \in U ; Q_{1} \neq Q_{2} \neq Q_{3} \neq Q_{1}$ e $\left|p-Q_{i}\right|<\epsilon$ para $i=1,2,3$, e os planos tangentes de $\Psi$ em $Q_{1}, Q_{2}, Q_{3}$ são paralelos.

2. $\forall \epsilon>0 \exists Q_{1}, Q_{2} \in U ; Q_{1} \neq Q_{2} e\left|p-Q_{i}\right|<\epsilon$ para $i=1,2$, e os planos tangentes de $\Psi$ em $Q_{1}, Q_{2}$ são iguais.

Demonstração: 1) Um plano $\xi$ é tangente à superfície $\Psi: U \rightarrow \mathbb{R}^{3}$ em $Q \in U$ se, e somente se, $Q$ é um ponto crítico da composição de $\Psi$ com a projeção ortogonal na reta $l$, onde $l$ é a reta que contém a origem e é normal a $\xi$.

Para um vetor unitário $v \in \mathbb{R}^{3}$, considere $\Pi_{v}: U \rightarrow \mathbb{R}$ a composição de $\Psi$ com a projeção ortogonal na reta gerada por $v$ :

$$
\Pi_{v}=\Psi(p) \cdot v
$$

Seja $v$ um normal unitário de $\Psi$ em $p$. Vamos provar que existem pontos $Q_{1}, Q_{2}, Q_{3}$ que são pontos críticos da função altura $\Pi_{w}$ para algum $w$ próximo de $v$. $p$ é uma cúspide de Gauss do mergulho $\Psi$ se, e somente se, $(v, p)$ é uma cúspide da aplicação catástrofe $\chi$ da família П. Para cada $w \in S^{2}, \chi^{-1}(w)$ é por definição o conjunto dos pontos críticos da função altura $\Pi_{w}$. Se (v,p) é uma cúspide de $\chi$ então existe $w$ arbitrariamente próximo de $v$ tal que $\chi^{-1}(w)$ tem três pontos, como ilustra a Figura 2.1.

Por outro lado se $\Psi \in A$, onde $A$ é o conjunto do Teorema 2.2.1, então o germe de $\chi$ no ponto (v,p) ou é regular, uma dobra, ou uma cúspide. Se o germe é regular, então $\chi^{-1}(w)$ tem um ponto para $\mathrm{w}$ próximo de $\mathrm{v}$, se o germe é uma dobra, então $\chi^{-1}(w)$ tem dois ou nenhum ponto para w próximo de v, como ilustra a Figura 2.2. Logo se 1) vale e a aplicação de Gauss é estável então $p$ é uma cúspide de Gauss.

2) Seja v o normal unitário de $\Psi$ em p. Queremos mostrar que existem pontos $Q_{1}$ e $Q_{2}$ pontos críticos da função $\Pi_{w}$ para algum w próximo de v, com $\Pi_{w}\left(Q_{1}\right)=\Pi_{w}\left(Q_{2}\right)$.

Seja $\chi$ a aplicação catástrofe da família $\Pi: S^{2} \times M^{2} \rightarrow S^{2} \times \mathbb{R}$. Pelo Lema 2.2.2, o germe de $\chi$ em (v,p) é uma cúspide (respectivamente, uma dobra, ou regular) se, e somente se o germe de $\Pi$ em (v,p) é um rabo de andorinha (respectivamente, uma cúspide, uma dobra). 


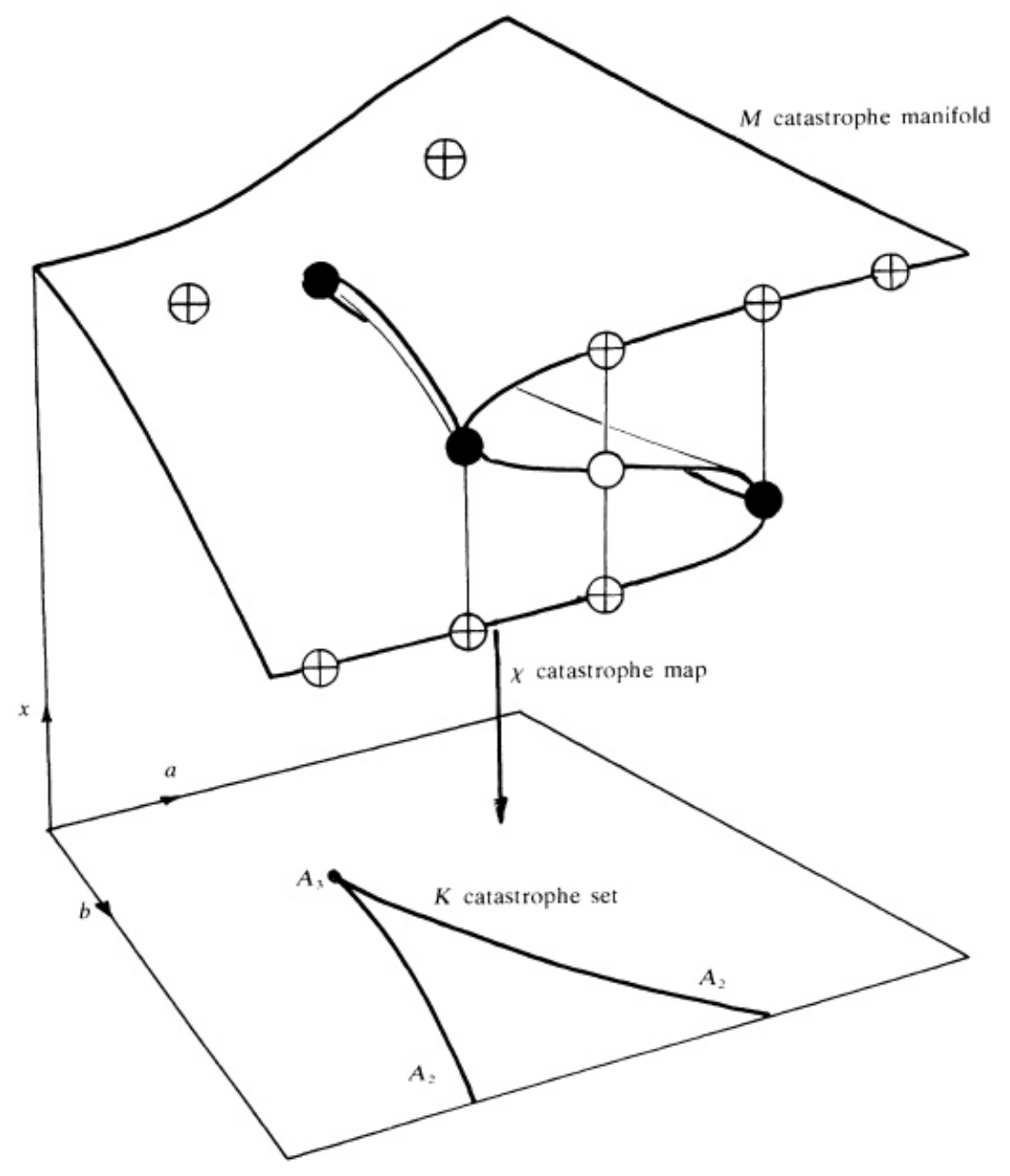

Figura 2.1: Cúspide na aplicação catástrofe.

A imagem do conjunto crítico de $\Pi$ próximo ao rabo de andorinha tem uma curva de pontos duplos, (Figura 2.9), isto é, existem duas curvas $\alpha, \beta:[0, \epsilon) \rightarrow C$ tal que $\alpha(0)=(v, p)=\beta(0), \alpha((0, \epsilon)) \cap \beta((0, \epsilon))=\emptyset \mathrm{e} \Pi(\alpha(t))=\Pi(\beta(t))$ para todo t em $[0, \epsilon)$.

Por outro lado, a imagem do conjunto crítico próximo a um ponto de dobra ou de cúspide não tem ponto duplo. Logo se 2) vale e a aplicação de Gauss é estável então $p$ é uma cúspide de Gauss. 


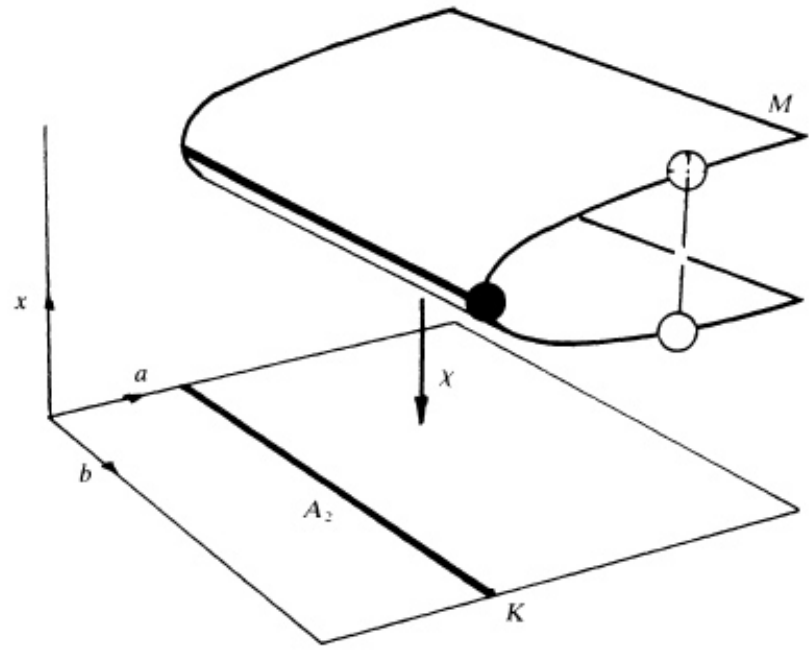

Figura 2.2: Dobra na aplicação catástrofe.
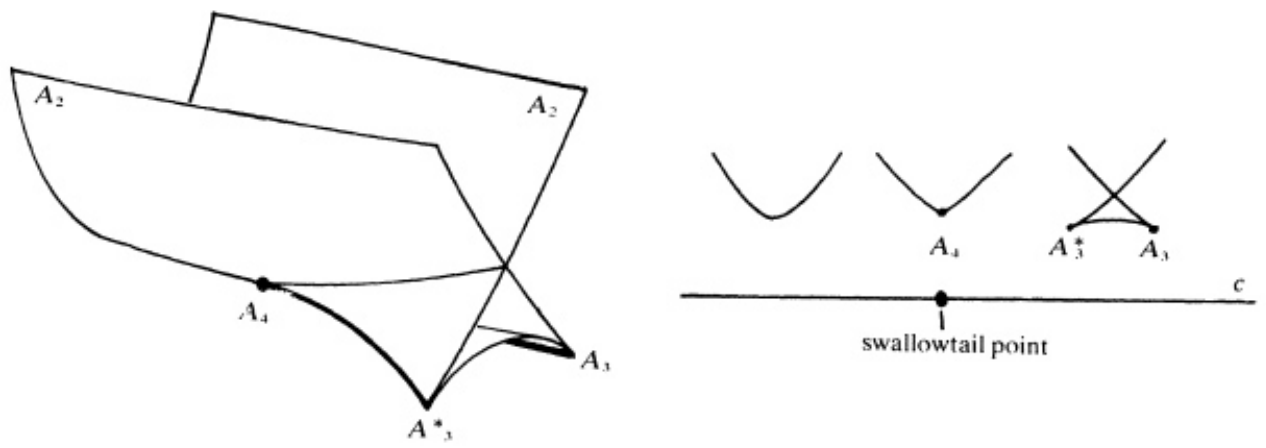

Figura 2.3: Rabo de andorinha na aplicação catástrofe.

\subsection{Sobre a classificação local das singularidades da função altura}

Nesta seção, vamos considerar o caso de uma superfície dada na forma de Monge, $(x, y, f(x, y))$, e comentar um pouco mais sobre estas singularidades que genericamente aparecem para a função $f$, ou seja, para a função altura (ver [8], [9]).

Para gerar as figuras que ilustram os tipos de singularidades ultilizamos o software superfíciesII de Montesinos (ver [35])

O tipo $A_{1}$ ocorre quando temos $a_{1}^{2} \neq 4 a_{0} a_{2}$ e então $f$ é uma singularidade de Morse em $(0,0)$. Assim temos que $f$ é equivalente a uma das seguintes funções 


$$
x^{2}+y^{2},-x^{2}-y^{2}, x^{2}-y^{2} .
$$

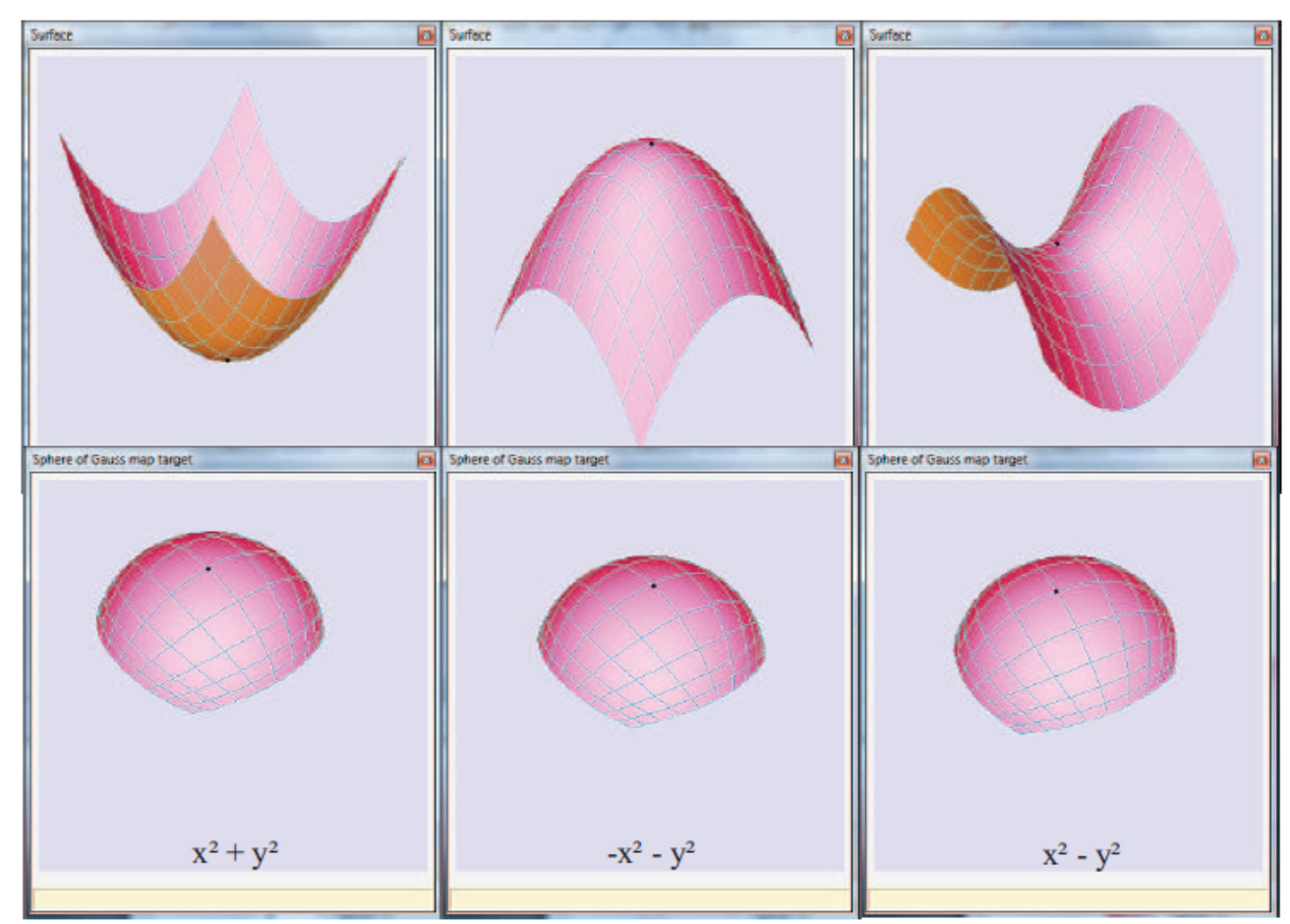

Figura 2.4: Singularidade $A_{1}$ localmente

Em particular o conjunto de zeros de $f$ (dado pela intersecção da superfície com o plano tangente na origem) é localmente difeomorfo ao conjunto de zeros de uma das seguintes funções $x^{2}+y^{2},-x^{2}-y^{2}, x^{2}-y^{2}$, isto é, é um ponto (dois primeiros casos) ou duas curvas que se interceptam transversalmente (terceiro caso).

Os pontos onde ocorrem singularidades $A_{1}$ da função altura $f$ são os pontos da superfície M onde a aplicação de Gauss é regular. Estes pontos são classificados como pontos elípticos $K(p)>0$ (dois primeiros casos) e pontos hiperbólicos $K(p)<0$ (terceiro caso).

O tipo $A_{2}$ aparece quando temos que $a_{1}^{2}=4 a_{0} a_{2}$ então a origem é um ponto parabólico $K(p)=0$ de M. A união de todos os pontos parabólicos nos dá o conjunto parabólico, que são os pontos de singularidades da aplicação de Gauss como visto na seção anterior. A superfície dada pela Equação 2.1 pode ser transformada por uma rotação em uma nova equação, que possui a seguinte forma (com um novo $a_{0}$ ), e é apresentada na Figura 2.5.

$$
z=f_{1}(x, y)=a_{0} x^{2}+b_{0} x^{3}+b_{1} x^{2} y+b_{2} x y^{2}+b_{3} y^{3}+\text { termos de ordem mais alta. }
$$


Se tivermos que $a_{0} \neq 0$, e $b_{3} \neq 0$, por um difeomorfismo na fonte temos que a função $f_{1}$ pode ser transformada em $\pm\left(x^{2}+y^{3}\right)$, assim temos que a interseção de $M$ com seu plano tangente é difeomorfo a $x^{2}+y^{3}=0$.

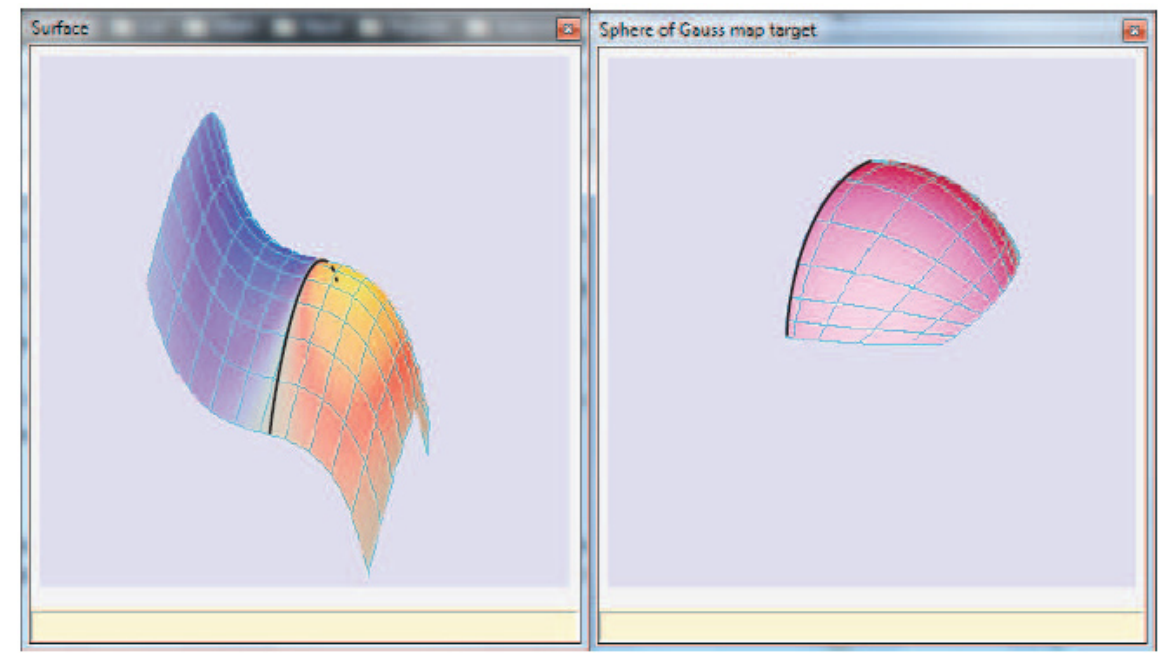

Figura 2.5: Singularidade $A_{2}$ (conjunto parabólico em preto) e sua imagem pela aplicação de Gauss

O tipo $A_{3}$ ocorre quando tivermos na equação $2.2 a_{0} \neq 0$ e $b_{3}=0$, então é necessário estudar os termos de ordem $4, c_{0} x^{4}+\ldots+c_{4} y^{4}$. "Completando quadrados" duas vezes podemos reescrever $f_{1}$ da forma $f_{1}(x, y)=a_{0} x^{2}+\left(c_{4}-b_{2}^{2} / 4 a_{0}\right) y^{4}+$ termos de ordem mais alta; e supondo que o coeficiente de $y^{4}$ é não-nulo, podemos eliminar os termos de ordem maior que 4 usando uma mudança de variáveis em $\mathrm{x}, \mathrm{y}$, obtendo $\pm x^{2} \pm y^{4}$. Quando os sinais são os mesmos escrevemos $A_{3}^{+}$(pois $x^{2}+y^{4}$ é $\mathcal{A}$-equivalente a $-x^{2}-y^{4}$ ) e quando estes diferem escrevemos $A_{3}^{-}$(pois $x^{2}-y^{4}$ é $\mathcal{A}$-equivalente a $-x^{2}+y^{4}$ ). A Figura 2.6 apresenta a singularidade $A_{3}^{+}$.

Além disso, se $b_{2} \neq 0$, temos que a origem é uma cúspide de Gauss e pode ser classificada de acordo com o seguinte:

Cúspide de Gauss Elíptica (positiva), $A_{3}^{+}: b_{2} \neq 0, a_{0} \neq 0, b_{3}=0, b_{2}^{2}<4 a_{0} c_{4}$. A interseção de M com seu plano tangente é (localmente) um único ponto.

Cúspide de Gauss Hiperbólica (negativa), $A_{3}^{-}: b_{2} \neq 0, a_{0} \neq 0, b_{3}=0 ; b_{2}^{2}>4 a_{0} c_{4}$. A interseção de M com seu plano tangente é (localmente) um par de curvas diferenciáveis tangentes. 


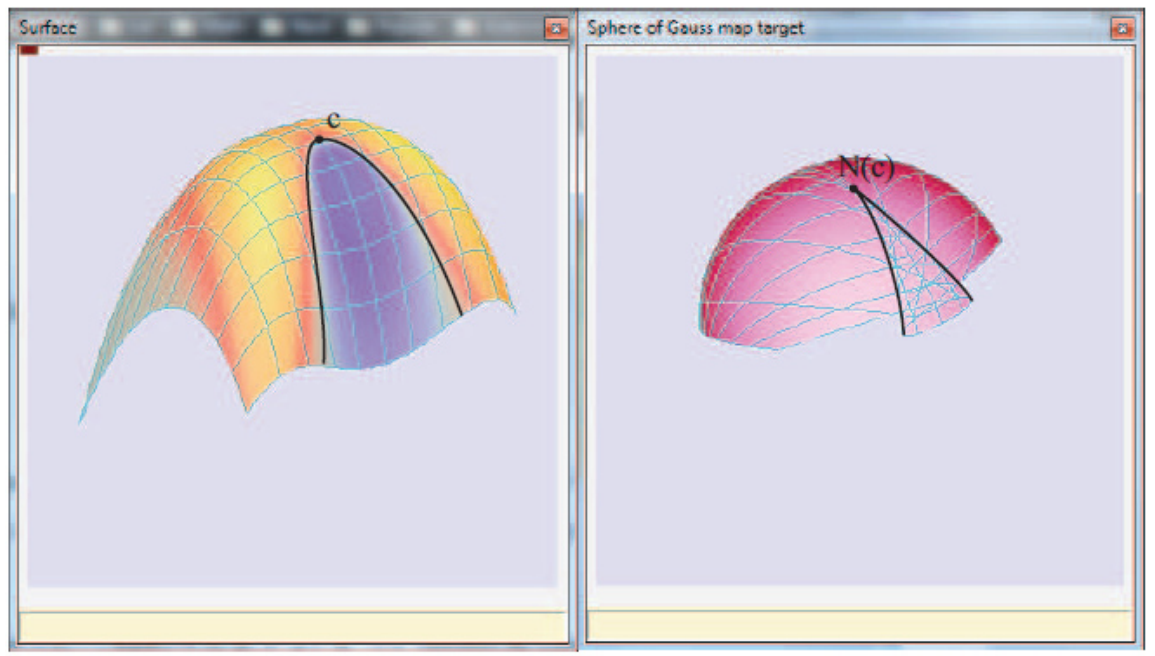

Figura 2.6: Singularidade $A_{3}^{+}$(ponto c) e sua imagem pela aplicação de Gauss

A condição $b_{2} \neq 0$ garante que o conjunto parabólico é suave na origem. O caso $b_{2}=0$ também é do tipo $A_{3}$, mas não ocorre em superfícies que tenham uma aplicação de Gauss estável. Veremos o caso $b_{2}=0$ na Seção 2.4 .

\subsection{Transições locais}

Na Seção 2.2, vimos os casos onde a aplicação de Gauss é estável. A seguir, veremos algumas famílias de superfícies a 1-parâmetro $t$ onde a aplicação de Gauss tem singularidade de $\mathcal{A}_{e}-\operatorname{codim}=1$ para $t=0$. Estamos interessados nas famílias de superfícies a 1 parâmetro, onde esse parâmetro varia em um intervalo fechado e limitado, e as superfícies para os parâmetros extremos desse intervalo possuem aplicação de Gauss estável. Essas famílias são chamadas de transições. Durante essas transições passamos por singularidades não estáveis de $\mathcal{A}_{e}-$ codim $=1$.

Em uma família de superfícies a 1-parâmetro, onde a aplicação de Gauss tem $\mathcal{A}_{e}-$ codim $=1$, temos as transições que acontecem devido a um contato maior entre a superfície e o plano tangente, chamados de contatos $A_{3}$ e $A_{4}$. Apresentaremos detalhes sobre as transições do tipo $A_{3}$ que são úteis para o desenvolvimento do trabalho, apresentaremos também transições do tipo $A_{4}$ (ver $\left.[8,9]\right)$.

Para gerar as figuras que ilustram as transições locais ultilizamos o software su- 
perfíciesII de Montesinos (ver [35])

\subsubsection{Transição $A_{3}$ : lábios/bicos}

Há quatro tipos de transições $A_{3}$, duas correspondendo a eventos do tipo "lábios" (Figura 2.7 (i) e (ii)) e duas correspondendo a eventos do tipo "bicos" (Figura 2.7 (iii) e (iv)). Todos esses casos são transições que levam ao nascimento/morte de um par de cúspides de Gauss. As cúspides resultantes serão ambas elípticas ou ambas hiperbólicas, dependendo do tipo de contato durante a transição que pode ser do tipo $A_{3}^{+}$ou $A_{3}^{-}$.

Agora, estudaremos o caso onde o contato entre a superfície e seu plano tangente é do tipo $A_{3}$ não versal. Estudaremos esse caso utilizando famílias de superfícies a 1parâmetro, ou seja, a família de superfícies $\Psi(x, y, t)=(x, y, f(x, y, t))$ onde funções $f(x, y, t)=x^{2}+\epsilon_{1} x^{2} y^{2}+\epsilon_{2} y^{4}+t y^{2}, \epsilon_{1}= \pm 1$ e $\epsilon_{2}= \pm 1$ nos dá todas as transições do tipo lábios/bicos da aplicação de Gauss $\mathcal{N}^{\prime}=\left(-f_{x},-f_{y}\right)$. A Figura 2.7 ilustra os tipos de transições $A_{3}$ e seus efeitos no conjunto parabólico, e também mostra as transições no conjunto de bifurcação na esfera. (ver[8] e [9]).

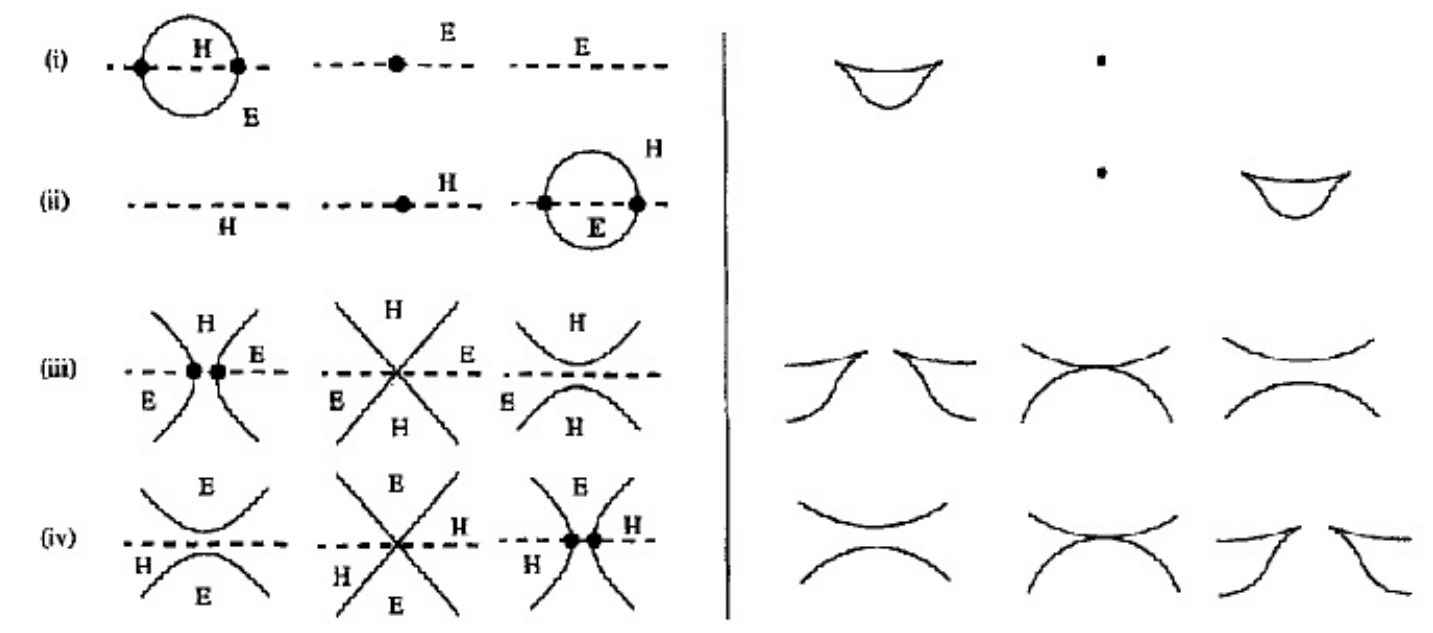

Figura 2.7: Transições do tipo $A_{3}$

Na Figura 2.7 temos que $\mathrm{H}$ significa que aquela região é do tipo hiperbólica, e E significa que aquela região é do tipo elíptica. Para $\epsilon_{2}=1$ temos uma transição do tipo $A_{3}^{+}$(cúspide de Gauss elípticas), para $\epsilon_{2}=-1$ temos uma transição do tipo $A_{3}^{-}$(cúspide 
de Gauss hiperbólicas); para $\epsilon_{1} \epsilon_{2}=1$ temos uma transição do tipo lábios na aplicação de Gauss, e para $\epsilon_{1} \epsilon_{2}=-1$ temos uma transição do tipo bicos na aplicação de Gauss.

Para $\epsilon_{2}=1$ e $\epsilon_{1}=1$ temos (i) na Figura 2.7, para $\epsilon_{2}=-1$ e $\epsilon_{1}=-1$ temos (ii) na Figura 2.7, para $\epsilon_{2}=-1$ e $\epsilon_{1}=1$ temos (iii) na Figura 2.7, e para $\epsilon_{2}=1$ e $\epsilon_{1}=-1$ temos (iv) na Figura 2.7.

As Figuras 2.8 e 2.9 ilustram uma transição do tipo lábios $\left(\epsilon_{1}=\epsilon_{2}=1\right)$ e uma transição do tipo bicos $\left(\epsilon_{1}=-\epsilon_{2}=-1\right)$, respectivamente.

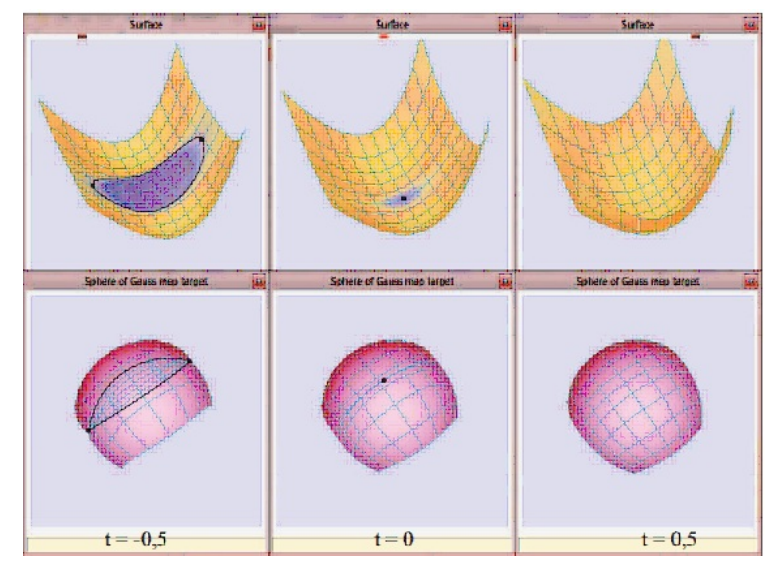

Figura 2.8: Transições do tipo lábios

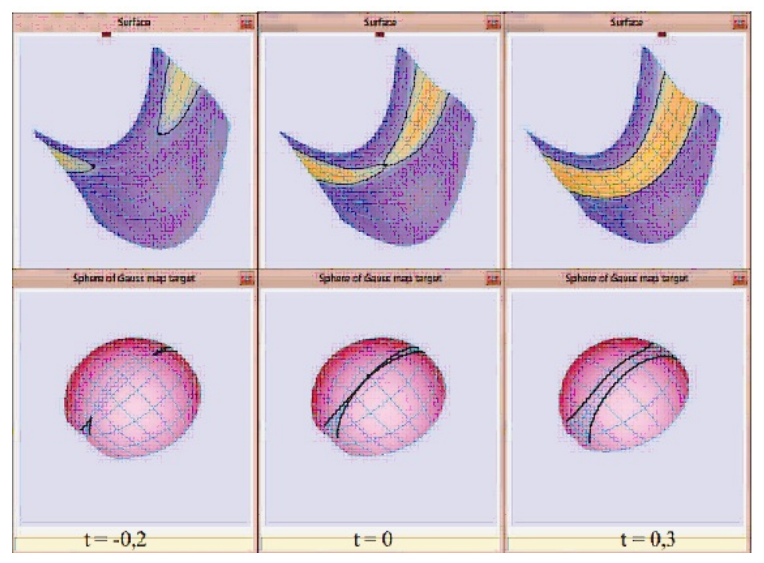

Figura 2.9: Transições do tipo bicos 


\section{Transições do tipo lábios}

Quando temos uma transição de Morse do tipo máximo ou mínimo na curva parabólica temos uma transição do tipo lábios na superfície. Podemos ter uma transição do tipo lábios em uma região de curvatura positiva (ou negativa), $\mathcal{R}_{1}$ de $\mathrm{M}$, e com isso surge uma nova região com curvatura negativa (resp. positiva). Com o surgimento dessa nova região temos o surgimento de uma fronteira entre essas regiões, essa fronteira é uma componente conexa do conjunto parabólico (curva) cuja imagem pela aplicação de Gauss é uma curva fechada com duas cúspides em $S^{2}$. No Capítulo 4, veremos o efeito dessa transição no grafo associado à aplicação de Gauss.

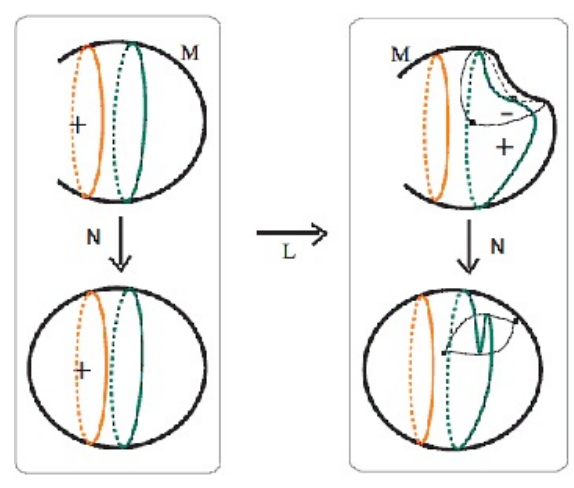

Figura 2.10: Transição do tipo lábios (L)

\section{Transições do tipo bicos}

Quando temos uma transição de Morse do tipo sela no conjunto parabólico temos uma transição do tipo bicos. Durante uma transição do tipo bicos dois arcos de um conjunto parabólico se aproximam até se juntarem em um ponto comum ("pontos do tipo bicos") e se separam em dois novos pares de arcos. Assim, podemos juntar(localmente) duas regiões elípticas dividindo(localmente) uma região hiperbólica, ou viceversa. Na Figura 2.10 vemos uma transição do tipo bicos que separa regiões hiperbólicas e junta regiões elípticas. Quando juntamos (localmente) duas regiões hiperbólicas e separamos uma região elíptica dada em duas partes temos uma transição $B_{+}$. Quando juntamos (localmente) duas regiões elípticas e separamos uma região hiperbólica dada em duas partes temos uma transição $B_{-}$. 


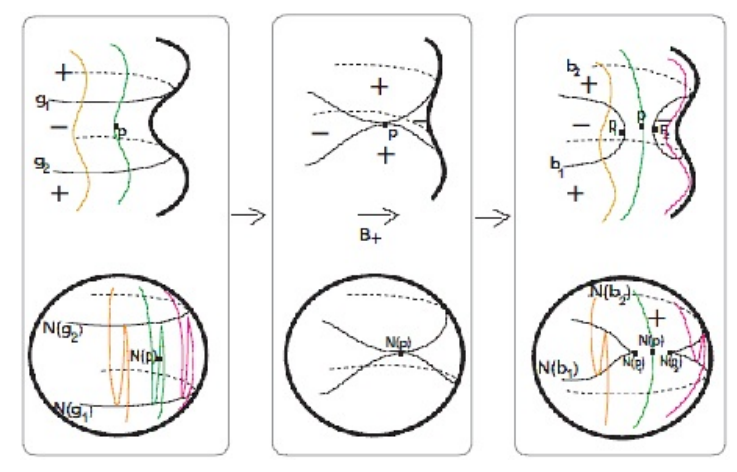

Figura 2.11: Transição do tipo bicos (B)

\subsubsection{Transição $A_{4}$.}

Aqui, o contato de M com o seu plano tangente é momentaneamente "pior" do que $A_{3}$. Neste caso há um único tipo de transição, com um par de cúspides de Gauss que aparecem (ou morrem), sendo uma cúspide elíptica e uma cúspide hiperbólica. A família de imagens de curvas parabólicas no âmbito da aplicação de Gauss (o conjunto bifurcação) sofre uma transição de rabo de andorinha.

A curva parabólica permanece suave durante toda a transição. O ridge (também suave) e a curva parabólica não tendo pontos de intersecção "antes", tornam-se tangencial no momento de transição e depois passa a ter dois pontos distintos onde a curva parabólica e o ridge se cruzam transversalmente, como vemos na Figura 2.12 (i). Para mais detalhes ver [8] e [9].

(i)

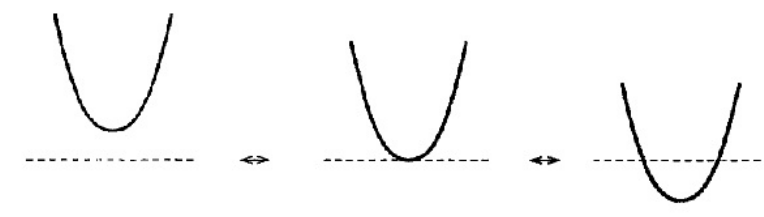

(ii)

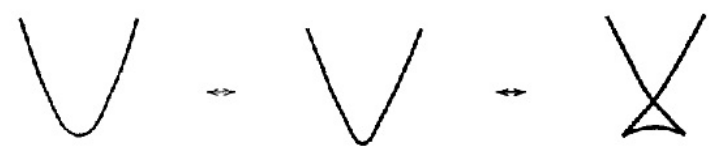

Figura 2.12: Transição $A_{4}$ : (i) efeito no conjunto parabólico (linha contínua = conjunto parabólico, linha pontilhada = ridge); (ii) efeito no conjunto bifurcação (imagem pela aplicação de Gauss do conjunto parabólico).

No germe de tipo $A_{4}$ temos que a interseção do plano tangente com a superfície nesses 
pontos é uma cúspide ramphoid, dada pela equação $x^{2}+y^{4}+y^{5}=0$ (Figura 2.13). Tais pontos representam o "nascimento ou morte" de duas cúspides de Gauss de sinais opostos. Por exemplo na família de superfícies $z=f(x, y, t)=x^{2}+2 x y^{2}+y^{4}+y^{5}+t y^{3}$ tem uma singularidade $A_{4}$ para $t=0$ e $x=y=0$. Temos algumas superfícies desta familía para $t$ fixos na Figura 2.14.

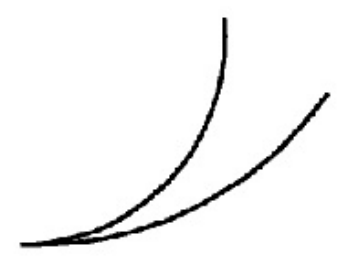

Figura 2.13: Cúspide ramphoid, interseção da superfície com seu plano tangente

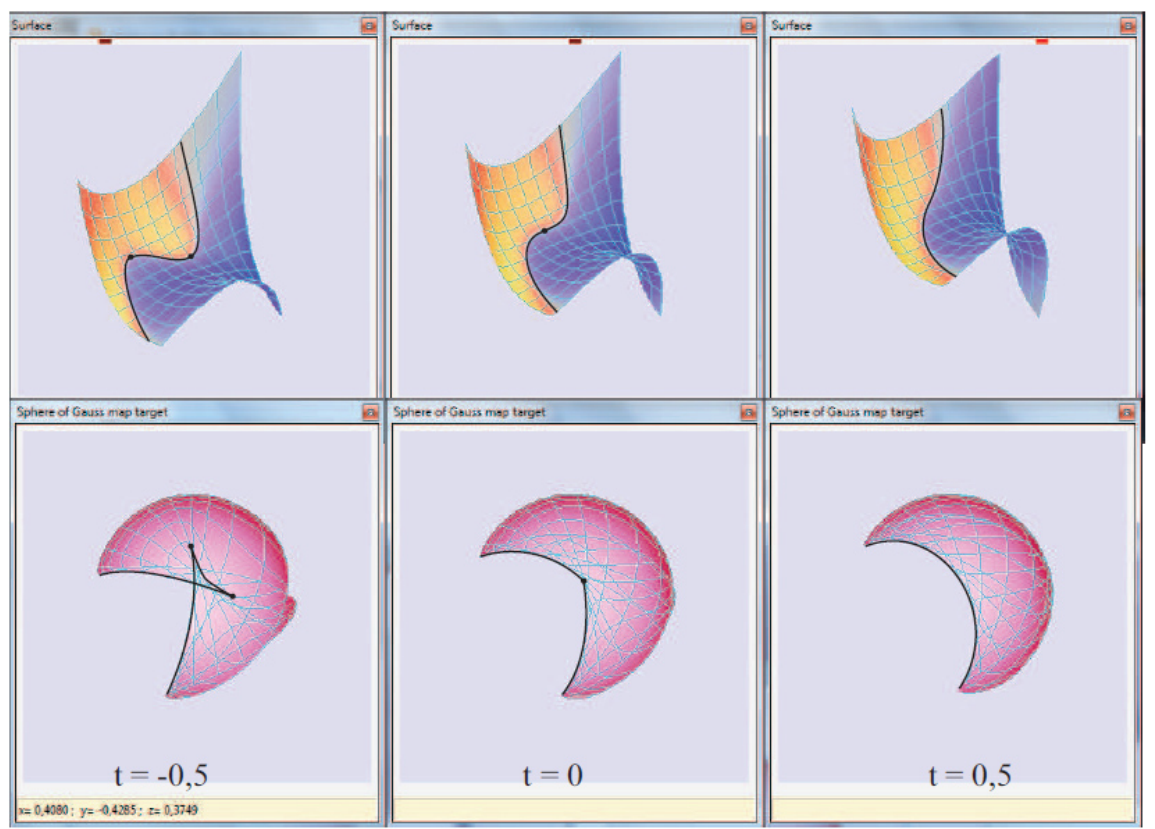

Figura 2.14: Transição $A_{4}$

Note que para usarmos a transição para reduzirmos o número de cúspides precisamos que exista um ponto duplo igual temos ilustrado na Figura 2.12. 


\subsection{Cirurgias da aplicação de Gauss}

Descreveremos a seguir algumas cirurgias em imersões de superfícies que alteram o conjunto singular das aplicações de Gauss de maneira conveniente. As cirurgias descritas aqui foram apresentadas em [30]. As cirurgias serão usadas para a construção dos resultados do Capítulo 4.

Denotaremos por $\mathcal{R}$ as regiões elípticas e por $\mathcal{H}$ as regiões hiperbólicas da superfície $M$.

\subsubsection{Cirurgia $S^{-}$}

Sejam $\mathcal{R}_{1}$ e $\mathcal{R}_{2}$ duas regiões elípticas e $\mathcal{H}$ um tubo hiperbólico. Removemos dois discos, um em cada região elíptica, e conectamos o tubo hiperbólico às suas fronteiras. Esse processo pode ser feito de uma maneira diferenciável. Com isso, acrescentamos duas novas componentes conexas, $\gamma_{1}$ e $\gamma_{2}$, ao conjunto parabólico.

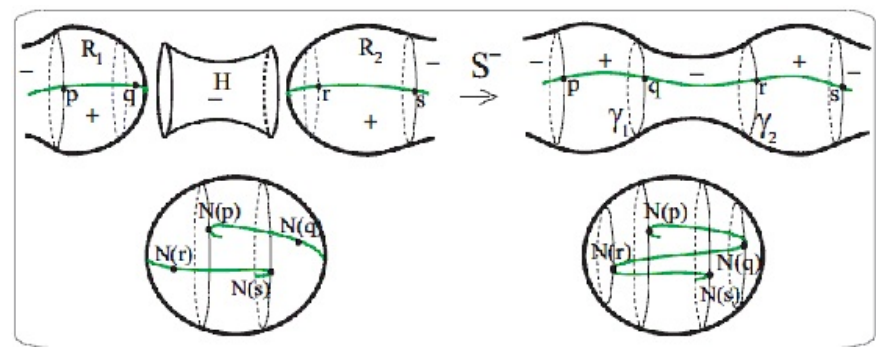

Figura 2.15: Cirurgia $S^{-}$e sua imagem pela aplicação de Gauss

Definição 2.5.1. Chamamos de cirurgia $S^{-}$o conjunto de passos descritos anteriormente que são ilustrados pela figura acima.

A cirurgia $S^{-}$pode ser feita nas regiões $\mathcal{R}_{1}$ e $\mathcal{R}_{2}$ que podem não ser necessariamente componentes conexas de uma mesma superfície. Suponha que $\mathcal{R}_{1}$ e $\mathcal{R}_{2}$ sejam regiões de superfícies $M_{1}$ e $M_{2}$, respectivamente. Como resultado da cirurgia obtemos uma nova superfície denotada por $\mathrm{M}=M_{1} \oplus^{-} M_{2}$ que além de ter todas as regiões conexas regulares de $M_{1}$ e $M_{2}$ tem uma nova região conexa com curvatura Gaussiana negativa e duas curvas parabólicas que separam a nova região das superfícies $M_{1}$ e $M_{2}$ ( ver Figura 2.15). Se 
$\mathcal{R}_{1}$ e $\mathcal{R}_{2}$ pertencem a uma mesma superfície $\mathrm{M}$ então temos que a cirurgia $S^{-}$aumenta o género da superfície $M$ em 1.

\subsubsection{Cirurgia $S^{-+}$}

Suponha que temos dois pedaços de superfícies $M_{i}, i=1,2$, ambos compostos de uma região elíptica $\mathcal{R}_{i}$, e uma região hiperbólica $\mathcal{H}_{i}$, separadas por uma curva parabólica $\gamma_{i}$ (i=1 para $M_{1}$ e i=2 para $M_{2}$ ). Aplicamos a cirurgia $S^{-}$unindo as duas regiões elípticas através de um cilindro hiperbólico $\mathcal{H}^{\prime}$. Isto introduz duas novas curvas parabólicas (fechadas) $\beta_{i}, i=1,2$ (na fronteira de $\mathcal{H}^{\prime}$ ). Utilizando uma transição do tipo bicos entre essas duas curvas fechadas, transformamos a região $\mathcal{H}^{\prime}$ em um disco no qual a fronteira é uma curva parabólica $\alpha$. Agora, aplicando duas transições do tipo bicos (entre $\alpha$ e $\gamma_{1}$ e $\alpha$ e $\gamma_{2}$ ) conectamos estes discos hiperbólicos simultaneamente com as regiões hiperbólicas $\mathcal{H}_{1}$ e $\mathcal{H}_{2}$, como apresentado na Figura 2.16

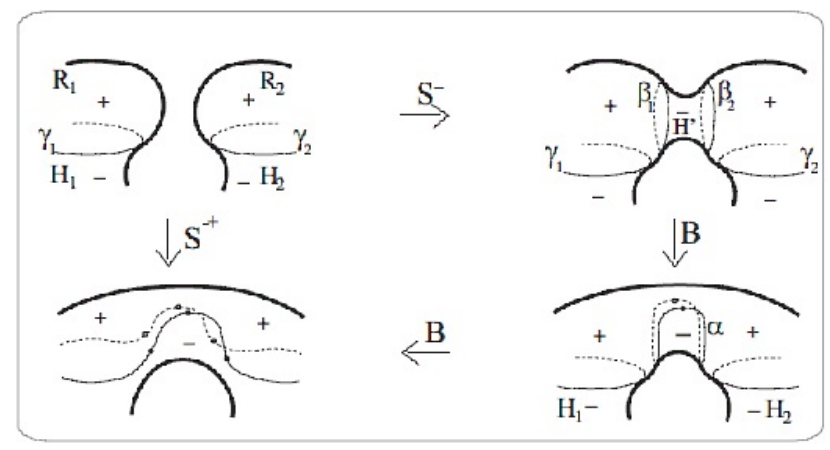

Figura 2.16: Cirurgia $S^{-+}$

Definição 2.5.2. Chamamos de Cirurgia $S^{-+}$o conjunto de passos descritos anteriormente que são ilustrados pela Figura 2.16

\subsubsection{Cirurgia $S^{+}$}

Sejam $\mathcal{R}_{1}$ e $\mathcal{R}_{2}$ duas regiões elípticas. Conectamos elas primeiramente com um tubo hiperbólico usando uma cirurgia do tipo $S^{-}$. Isso introduz duas curvas parabólicas novas, as quais são fronteiras da nova região hiperbólica $\mathcal{H}$. Agora aplicamos uma transição 
do tipo bicos unindo estas duas curvas parabólicas e transformando $\mathcal{H}$ em um disco. Finalmente, fazemos esse disco desaparecer utilizando uma transição do tipo lábios.

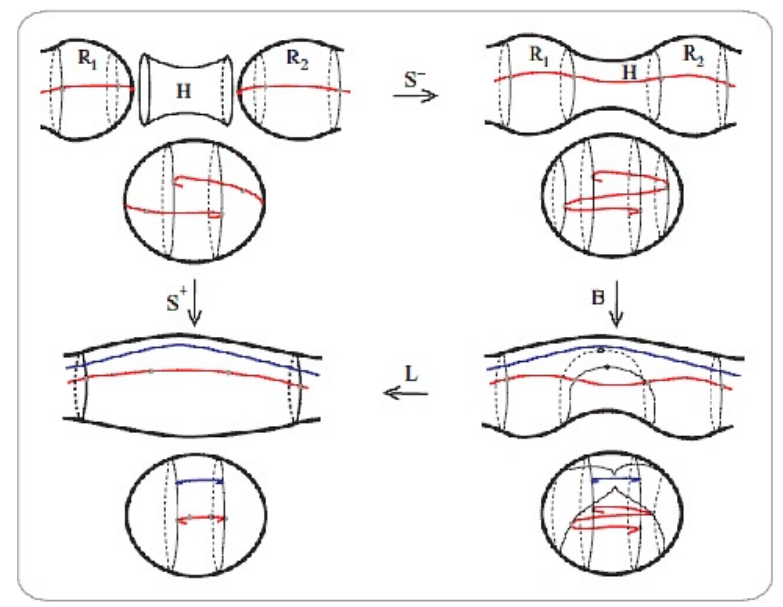

Figura 2.17: Cirurgia $S^{+}$e sua imagem pela aplicação de Gauss

Definição 2.5.3. Chamamos de Cirurgia $S^{+}$o conjunto de passos descritos anteriormente que são ilustrados pela Figura 2.17. 


\section{Capítulo 3}

\section{Invariantes de aplicações estáveis}

Neste capítulo, apresentaremos um invariante topológico (global) para aplicações estáveis de $C^{\infty}\left(M, S^{2}\right)$. Apresentaremos, primeiramente, uma técnica de associar grafos às superfícies com um conjunto de curvas prefixado. Os grafos associados às superfícies carregam informações da topologia das superfícies e de certo modo podem caracterizá-las totalmente. Temos que o conjunto singular, $\Sigma f$, de uma aplicação estável $f \in C^{\infty}\left(M, S^{2}\right)$ é formado por curvas fechadas e disjuntas na superfície $M$ (Lema 1.3.27). Com isso, aplicamos a técnica de associar grafos à superfície e seu conjunto singular, ou seja, associamos um grafo ao $\operatorname{par}(M, \Sigma f)$. Veremos que os grafos associados aos pares da forma $(M, \Sigma f)$ são invariantes globais para aplicações estáveis. As Figuras deste capítulo foram tiradas dos trabalhos [6,33].

\subsection{Grafos associados às superfícies com um conjunto de curvas prefixado}

Iniciaremos apresentando uma técnica de associar grafos (com peso) às superfícies com curvas prefixadas. Considere $M$ uma superfície (compacta, orientável e sem bordo) e $\mathcal{C}$ um conjunto finito de curvas fechadas e disjuntas em $M$. O conjunto $\mathcal{C}$ divide a superfície $M$ em um conjunto de regiões conexas, isto é, o complemento $M-\mathcal{C}$ é a união disjunta de regiões conexas. 
Definição 3.1.1. [33] Associamos um grafo $\mathcal{G}$ ao par $(M, \mathcal{C})$ do seguinte modo:

- a cada região conexa do complemento de $\mathcal{C}$ em $M$, associamos um vértice,

- a relação de adjacência entre os vértices é dada em função da adjacência das regiões, ou seja, dois vértices estão unidos por arestas sempre que as regiões correspondentes forem adjacentes,

- o número de arestas conectando dois vértices é dado pelo númeto de componentes de $\mathcal{C}$ que separam as duas regiões correspondentes,

- Laços ocorrem quando uma curva é componente de bordo de uma única região,

- o peso em cada vértice corresponde ao género da respectiva região,

- a soma total dos pesos em $\mathcal{G}$ é denotada por $W(\mathcal{G})$.

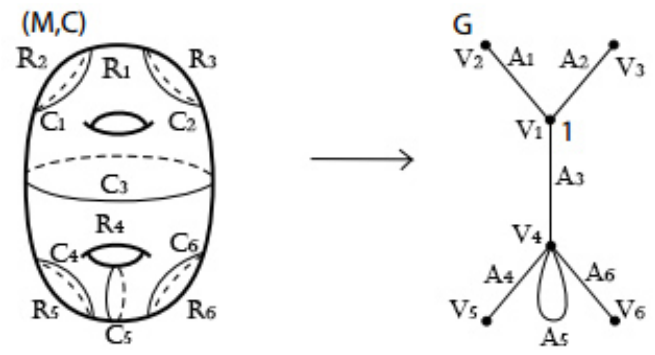

Figura 3.1: Grafo associado ao 2-toro com um conjunto de curvas prefixado.

Na Figura 3.1 temos que a superfície $M$ está dividida em seis regiões, e apenas a região $R_{1}$ possui género não nulo, igual a 1 . Temos a existência de um laço, a aresta $A_{5}$ no vértice $V_{4}$, pois a curva $C_{5}$ é representada por essa aresta que está separando duas partes da região $R_{4}$.

Definição 3.1.2. [33] Sejam $\mathcal{C}$ e $\mathcal{C}^{\prime}$ conjuntos de curvas sobre duas superfícies $M$ e $M^{\prime}$, respectivamente. Os pares $(M, \mathcal{C})$ e $\left(M^{\prime}, \mathcal{C}^{\prime}\right)$ são ditos equivalentes quando existe um difeomorfismo de $M$ em $M^{\prime}$ que leva $\mathcal{C}$ em $\mathcal{C}^{\prime}$.

Lema 3.1.3. [33] $S e(M, \mathcal{C})$ e $\left(M^{\prime}, \mathcal{C}^{\prime}\right)$ são equivalentes, então os grafos dos respectivos pares são isomorfos. 


\subsection{GRAFOS ASSOCIADOS ÀS SUPERFÍCIES COM UM CONJUNTO DE CURVAS PREFIXADO57}

Demonstração: Seja $\psi: M \rightarrow M^{\prime}$ um difeomorfismo tal que $\psi(\mathcal{C})=\mathcal{C}^{\prime}$ e sejam $\mathcal{G}$ e $\mathcal{G}^{\prime}$ grafos associados, respectivamente, aos pares $(M, \mathcal{C})$ e $\left(M^{\prime}, \mathcal{C}^{\prime}\right)$. Provaremos que $\mathcal{G}$ e $\mathcal{G}^{\prime}$ são isomorfos, vamos induzir esse isomorfismo a partir da $\psi$. Para isso vamos inicialmente ver que estes dois grafos têm o mesmo número de arestas. Como $\psi$ é um difeomorfismo tal que $\psi(\mathcal{C})=\mathcal{C}^{\prime}$, temos que as componentes conexas de $\mathcal{C}$, isto é, as curvas destes conjuntos, são levadas biunivocamente nas componentes conexas de $\mathcal{C}^{\prime}$. Logo, temos que o número de curvas em $\mathcal{C}$ e $\mathcal{C}^{\prime}$ são iguais. Sendo o número de arestas do grafo igual ao número de componentes conexas do conjunto de curvas, temos que $\mathcal{G}$ e $\mathcal{G}^{\prime}$ têm o mesmo número de arestas.

Agora vamos provar que $\mathcal{G}$ e $\mathcal{G}^{\prime}$ têm o mesmo número de vértices. De fato, sendo $\psi$ bijetora, temos

$$
\begin{aligned}
\psi(M-\mathcal{C}) & =\psi(M)-\psi(\mathcal{C}) \\
& =M^{\prime}-\mathcal{C}^{\prime} .
\end{aligned}
$$

Logo cada componente conexa de $M-\mathcal{C}$ é levada biunivocamente numa componente conexa de $M^{\prime}-\mathcal{C}^{\prime}$, pelo difeomorfismo $\psi$. Portanto, temos que o número de regiões do complemento $M-\mathcal{C}$ é igual ao número de regiões do complemento $M^{\prime}-\mathcal{C}^{\prime}$. Como o número de vértices de um grafo associado corresponde ao número de regiões do complemento do conjunto de curvas, temos que $\mathcal{G}$ e $\mathcal{G}^{\prime}$ possuem o mesmo número de vértices.

Vamos mostrar que $\mathcal{G}$ e $\mathcal{G}^{\prime}$, têm a mesma estrutura de adjacências. Sejam $\mathcal{R}_{1}$ e $\mathcal{R}_{2}$, duas regiões adjacentes, do complemento $M-\mathcal{C}$, separadas por uma curva $\alpha$. Sabemos que $\mathcal{R}_{1} \cup \mathcal{R}_{2} \cup \alpha \subset M$ é conexa por caminhos, portanto $\psi\left(\mathcal{R}_{1}\right) \cup \psi\left(\mathcal{R}_{2}\right) \cup \psi(\alpha)=\psi\left(\mathcal{R}_{1} \cup\right.$ $\left.\mathcal{R}_{2} \cup \alpha\right) \subset M^{\prime}$ também é conexa por caminhos, e $\psi\left(\mathcal{R}_{1}\right)$ e $\psi\left(\mathcal{R}_{2}\right)$ são regiões adjacentes de $M^{\prime}-\mathcal{C}^{\prime}$. Portanto, $\psi$ preserva as adjacências das regiões. Logo, $(M, \mathcal{C})$ e $\left(M^{\prime}, \mathcal{C}^{\prime}\right)$, e consequentemente $\mathcal{G}$ e $\mathcal{G}^{\prime}$, têm a mesma estrutura de adjacências.

Para finalizar, note que $\psi$ leva difeomorficamente as regiões de $M-\mathcal{C}$ nas regiões de $M^{\prime}-\mathcal{C}^{\prime}$, portanto temos que os vértices correspondentes têm o mesmo peso, uma vez que o género de uma região é preservado por homeomorfismos, e os difeomorfismos são homeomorfismos também. 


\subsection{Grafos como invariantes de aplicações estáveis}

Seja $f \in C^{\infty}\left(M, S^{2}\right)$ uma aplicação estável. Do Lema 1.3.27 temos que o conjunto singular $\Sigma f$ consiste de curvas fechadas e disjuntas em $M$. Logo podemos associar um grafo à aplicação $f, \mathcal{G}_{f}$, como sendo o grafo associado ao par $(M, \Sigma f)$, tomando o conjunto $C=\Sigma f$.

A cada aplicação estável $f \in C^{\infty}\left(M, S^{2}\right)$ conseguimos um grafo associado $\mathcal{G}_{f}$. Vamos provar que $\mathcal{G}_{f}$ é um grafo bipartido e é um invariante por $\mathcal{A}$-equivalência. O grafo $\mathcal{G}_{f}$ carrega informações da aplicação, da superfície, do número de componentes conexas de $\Sigma f$, bem como o tipo topológico de seu complemento em $M$.

Proposição 3.2.1. [6] Se $f: M \rightarrow S^{2}$ é uma aplicação estável, então $\mathcal{G}_{f}$ é bipartido, onde $\mathcal{G}_{f}$ é o grafo associado ̀̀ $f$.

Demonstração: Seja $f: M \rightarrow S^{2}$ uma aplicação estável e $\mathcal{G}_{f}$ seu grafo associado. Da Definição 1.3.25 temos que cada região do complemento $M-\mathcal{C}$ recebe um sinal \pm . Logo temos uma maneira natural de atribuir sinais aos vértices de $\mathcal{G}_{f}$, onde cada vértice recebe o sinal da região correspondente. Temos que cada curva de $\Sigma f$ separa regiões de sinais opostos, logo cada aresta de $\mathcal{G}_{f}$ conecta vértices de sinais opostos. Portanto, $\mathcal{G}_{f}$ é bipartido.

Teorema 3.2.2. [33] O grafo de uma aplicação estável é invariante por $\mathcal{A}$-equivalência. Em particular, é invariante por isotopia estável.

Demonstração: Sejam $f, g \in C^{\infty}\left(M, S^{2}\right)$ duas aplicações $\mathcal{A}$ - equivalentes e sejam $l$ : $M \rightarrow M$ e $k: S^{2} \rightarrow S^{2}$ difeomorfismos tais que $f=k^{-1} \circ g \circ l$.

Para mostrar que os grafos são invariantes por $\mathcal{A}$-equivalência, basta mostrar que $\mathcal{G}_{f}$ é equivalente a $\mathcal{G}_{g}$. Dado $p \in \Sigma f$, temos que $(d f)_{p}$ não é injetora. Pela regra da cadeia $(d f)_{p}=\left(d k^{-1}\right)_{g(l(p))} \circ(d g)_{l(p)} \circ(d l)_{p}$. Como $\left(d k^{-1}\right)_{g(l(p))}$ e $(d l)_{p}$ são isomorfismos, temos que $(d g)_{l(p)}$ não é injetora. Assim, $l(p) \in \Sigma g$. Portanto, $l$ é um difeomorfismo de $M$ em $M$ que leva $\Sigma f$ em $\Sigma g$, ou seja, os pares $(M, \Sigma f)$ e $(M, \Sigma g)$ são equivalentes. Logo, pelo Lema $3.1 .3, \mathcal{G}_{f}$ e $\mathcal{G}_{g}$ são isomorfos. 


\subsection{Invariantes locais de aplicações estáveis}

Ohmoto e Aicardi em [42], estudaram alguns invariantes locais para o contorno aparente de aplicações estáveis de superfícies fechadas no plano (imagem do conjunto singular), que são o número de cúspides e o número de pontos duplos, respectivamente $I_{C}$ e $I_{D}$. Hacon, Mendes de Jesus e Romero-Fuster apresentaram em [14] outro invariante, $I_{E}$, que é o número de componentes conexas do contorno aparente de uma aplicação estável de superfície no plano. Em [25], vimos que esses invariantes podem ser obtidos para aplicações estáveis de superfícies na 2-esfera, já que a 2-esfera é localmente um plano. Ou seja, podemos definir estes invariantes para aplicações de Gauss estáveis.

Para homotopias entre duas aplicações estáveis podem ocorrer transições. Uma transição ocorre quando a homotopia passa por uma aplicação não estável. Na Figura 3.2, vemos transições convenientes para o estudo do número de cúspides e do número de componentes conexas da superfície. Diremos que a orientação é positiva (negativa) se a transição aumentar (diminuir) o número de pontos duplos ou aumentar (diminuir) o número de cúspides.
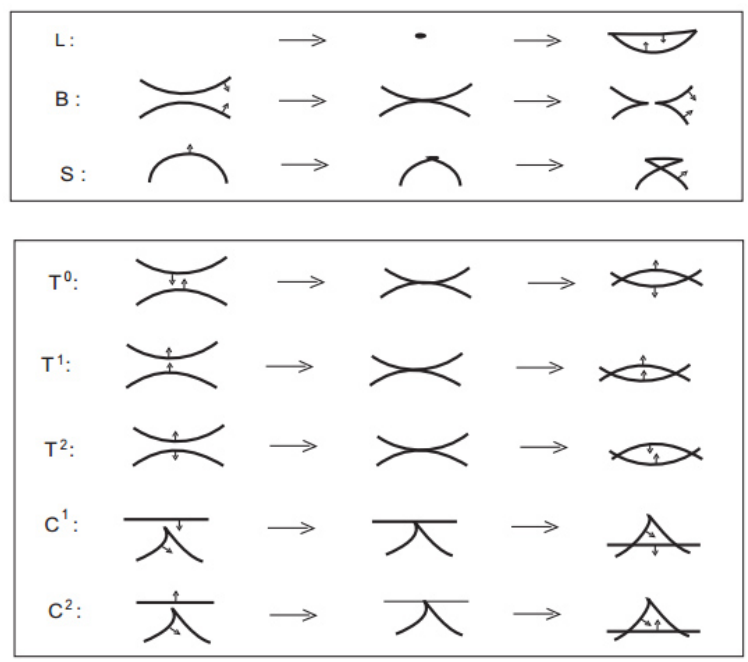

Figura 3.2: [42] Alguns tipos de transições.

As transições na Figura 3.2 são denominadas lábios $(L)$, bicos $(B)$, rabo de andorinha $(S)$, tangência de dobras $\left(T^{0}\right),\left(T^{1}\right)$ e $\left(T^{2}\right)$, dobra com cúspides $(C 1)$ e $(C 2)$. Podemos classificar essas transições de acordo com o tipo de alteração que elas fazem no 
conjunto singular e no contorno aparente de uma aplicação. As transições do tipo $(L)$ e $(B)$ alteram o número de cúspides, e alteram o número de componentes do conjunto singular; a transição do tipo $(S)$ altera o número de cúspide, e o número de pontos duplos; as transições do tipo $\left(T^{0}\right),\left(T^{1}\right),\left(T^{2}\right),(C 1)$ e $(C 2)$ alteram o número de pontos duplos.

Em cada linha da Figura 3.2, representamos localmente três momentos de uma transição e a imagem do contorno aparente destas três aplicações em $C^{\infty}\left(M, \mathbb{R}^{2}\right)$. Os momentos da direita e da esquerda são estáveis, já a aplicação do meio não é estável. As setas no contorno aparente apontam para onde temos que o número de pré-imagens aumenta (por dois). O índice $i$ em $\left(T^{i}\right)$ e $\left(C^{i}\right)$ vem da metade do número de pré-imagens (zero, dois ou quatro) dentro da nova região criada após a transição do tipo tangência.

Os invariantes $I_{C}, I_{D}$ e $I_{E}$, são os números de pontos de cúspides, pontos de dobra e componentes conexas do contorno aparente, respectivamente. Vemos que as transições $(L),(B)$ e $(S)$ alteram o número de cúspides por \pm 2 , dependendo do sentido que fazemos a transição, e as transições do tipo $\left(T^{i}\right)$ e $\left(C^{i}\right)$ alteram o número de pontos duplos por \pm 2 . Já a transição do tipo $(S)$ altera por \pm 1 o número de pontos duplos, dependendo do sentido que fazemos a transição.

Uma homotopia (transição) entre as aplicações estáveis $f_{0}$ e $f_{1}$ pode ser vista como uma família $F_{t}$ de aplicações a 1 -parâmetro $t,-1 \leq t \leq 1$, tal que $F_{-1}=f_{0}$ e $F_{1}=f_{1}$. Para o caso da aplicação de Gauss vimos que uma forma de fazer essa homotopia é através das transições.

Em [42], temos o seguinte resultado que relaciona o número de cúspides e o número de pontos duplos com a quantidade de transições dos tipos: $(L),(B),(S),\left(T^{0}\right),\left(T^{1}\right),\left(T^{2}\right)$, $(C 1)$ e $(C 2)$.

Teorema 3.3.1. [42] A variação do número de cúspides e do número de pontos duplos durante uma homotopia entre as aplicações $f_{0}$ e $f_{1}$, são dadas por

$$
\begin{aligned}
& \text { 1. } \Delta I_{C}=I_{C}\left(f_{1}\right)-I_{C}\left(f_{0}\right)=2(\Delta L+\Delta B+\Delta S) \\
& \text { 2. } \Delta I_{D}=I_{D}\left(f_{1}\right)-I_{D}\left(f_{0}\right)=2(\Delta T+\Delta C)+\Delta S \text {, }
\end{aligned}
$$

onde $\Delta L$ é o número de transições com orientação positiva menos o número de transições com orientação negativa do tipo lábios, $\Delta B$ é o número de transições com orientação posi- 
tiva menos o número de transições com orientação negativa do tipo bicos, $\Delta S$ é o número de transições com orientação positiva menos o número de transições com orientação negativa do tipo rabo de andorinha, $\Delta T$ é o número de transições com orientação positiva menos o número de transições com orientação negativa do tipo tangência de dobra e $\Delta C$ é o número de transições com orientação positiva menos o número de transições com orientação negativa do tipo tangência de dobra com cúspide. As orientações positivas sofridas durante a homotopia sâo representadas na Figura 3.2, onde a orientação é positiva se a transição aumentar o número de pontos duplos ou aumentar o número de cúspides.

Neste trabalho, também estudamos o invariante $I_{C}$ para transições de aplicações de Gauss estáveis (ver Subseção 4.4.3).

\subsubsection{Relação entre cúspides e característica de Euler}

Nesta subseção, veremos uma demonstração da fórmula de Quine [41], no caso de aplicações de superfícies na esfera (Teorema 3.3.6). Neste caso, a fórmula estabelece uma relação entre os sinais das cúspides de uma aplicação e a característica de Euler da superfície. Este resultado é um caso particular do teorema encontrado em [41] (ver também [6]).

O Corolário do Teorema de Quine será usado na Subseção 4.4.3. Esse corolário nos dá a soma dos sinais das cúspides, uma vez que temos que essa soma depende da característica de Euler da superfície e do grau da aplicação de Gauss, onde segundo [23] o grau da aplicação de Gauss de uma superfície é dado em função do seu género.

Seja $f: M \rightarrow N$ uma aplicação estável, onde $M$ e $N$ são duas superfícies suaves, orientadas. Pela Definição 1.3.25, cada região do complemento $M \backslash \Sigma f$ recebe um sinal \pm . Como já vimos anteriormente, podemos associar a um ponto de cúspide $C$ de $f$, um sinal $s(C)= \pm 1$ definido de acordo com a região para a qual a cúspide aponta.

Teorema 3.3.2. (Teorema de Quine). Sejam $M$ e $N$ duas 2-variedades suaves, compactas, orientadas e conexas, $f: M \rightarrow N$ uma aplicação estável, $M^{+}$o fecho do conjunto dos pontos regulares nos quais $f$ preserva a orientação, $M^{-}$o fecho dos pontos regulares os quais $f$ inverte a orientação e $C_{1}, \ldots, C_{n}$ pontos de cúspides, então

$$
\chi(M)-2 \chi\left(M^{-}\right)+\Sigma s\left(C_{i}\right)=\chi(N) \operatorname{deg}(f),
$$




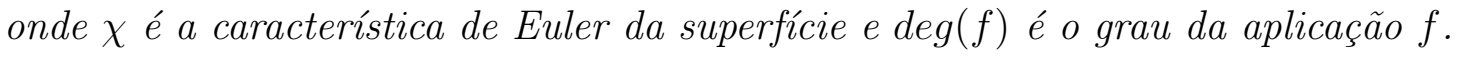

Temos que as transições do tipo lábios ou bicos alteram a soma dos sinais das cúspides, denotada por $\Sigma s\left(C_{i}\right)$, por \pm 2 , enquanto a transição do tipo rabo de andorinha mantém o valor de $\Sigma s\left(C_{i}\right)$.

Na Figura 3.3, vemos que as cúspides obtidas pelas transições do tipo lábios ou bicos têm o mesmo sinal, enquanto as cúspides obtidas pelas transições do tipo rabo de andorinha possuem sinais opostos.

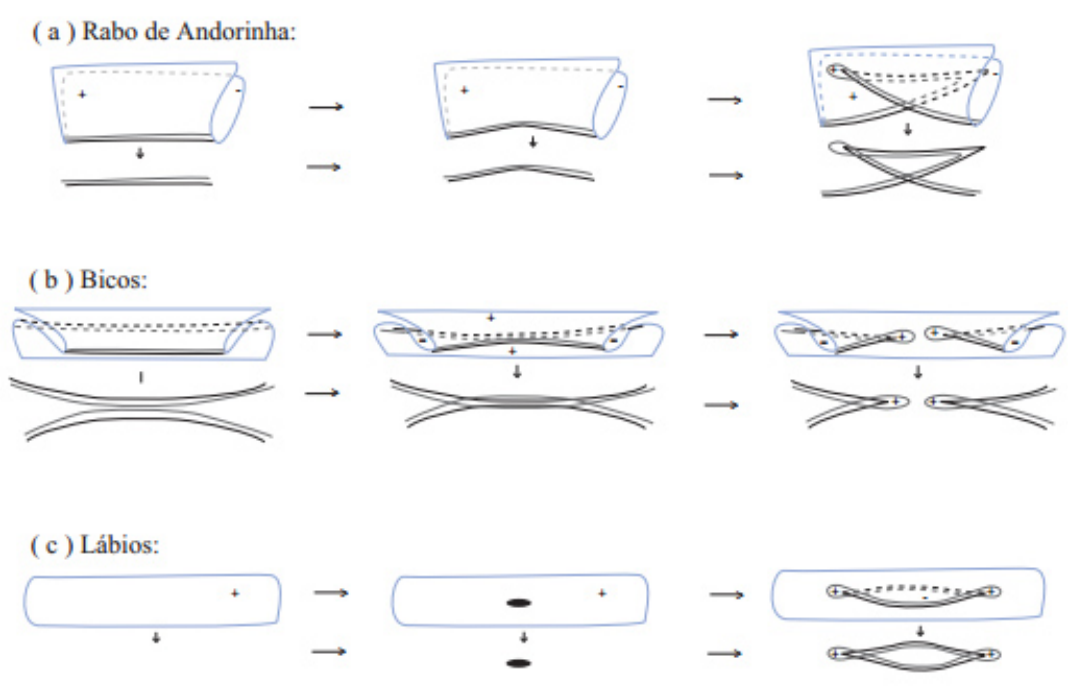

Figura 3.3: [6] Sinais das cúspides.

Os próximos lemas serão usados para provar o corolário do Teorema de Quine.

Lema 3.3.3. [6] Sejam $f_{0}$ e $f_{1}$ aplicações estáveis de superfícies fechadas e orientadas na esfera, onde $f_{1}$ é obtida por $f_{0}$, a partir de uma transição do tipo bicos ou lábios. Se $M_{0}$ e $M_{1}$ são, respectivamente, o complemento dos conjuntos singulares $\Sigma f_{0}$ e $\Sigma f_{1}$, então

$$
\chi\left(M_{1}^{ \pm}\right)=\chi\left(M_{0}^{ \pm}\right) \mp s(C),
$$

onde $s(C)$ denota o sinal das duas novas cúspides resultantes da transição do tipo bicos ou lábios.

Demonstração: Sejam $M_{0}^{+}\left(M_{0}^{-}\right)$o fecho da região positiva (negativa) de $f_{0}$ e $M_{1}^{+}\left(M_{1}^{-}\right)$ o fecho da região positiva (negativa) de $f_{1}$, onde $M_{0}$ e $M_{1}$ são, respectivamente, o complemento dos conjuntos singulares $\Sigma f_{0}$ e $\Sigma f_{1}$. Vamos primeiro analisar a transição do 
tipo bicos $(B)$. Para este caso, temos duas possibilidades: $\left(B^{+}\right)$e $\left(B^{-}\right)$, que respectivamente, acrescentam e diminuem o número de componentes conexas do contorno aparente da aplicação.

Vamos considerar cada uma delas separadamente.

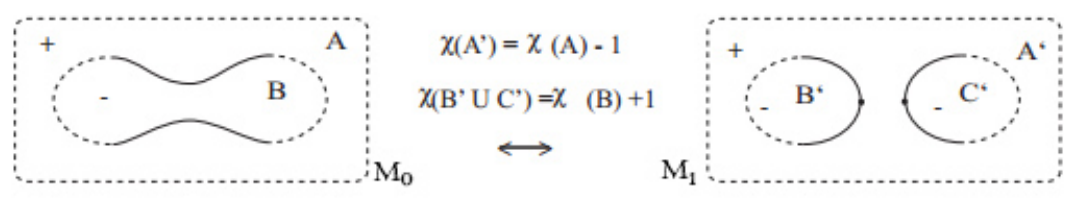

Figura 3.4: [6] Alterando a característica de Euler pela transição $\left(B^{+}\right)$.

Na Figura 3.4, vemos a transição do tipo $\left(B^{+}\right)$. No começo, temos uma componente de $\Sigma f_{0}$, que separa as regiões $\mathbf{A}$ e $\mathbf{B}$ de $M_{0}$. Após a transição $\left(B^{+}\right)$, a componente de $\Sigma f_{0}$ que separa as regiões $\mathbf{A}$ e $\mathbf{B}$ de $M_{0}$ é dividida em duas, assim, temos que a região A ganha mais uma componente com bordo e passa a ser denotada por $\mathbf{A}^{\prime}$. A região $\mathbf{B}$ é dividida em duas novas regiões que denotamos por $\mathbf{B}^{\prime}$ e $\mathbf{C}^{\prime}$.

Como a região $\mathbf{A}^{\prime}$ tem uma componente de bordo a mais que $\mathbf{A}$, pela Proposição 1.1.25 temos que

$$
\chi\left(A^{\prime}\right)=\chi(A)-1
$$

para $s(C)=1$ (caso onde a região $\mathbf{A}$ é positiva) temos

$$
\chi\left(A^{\prime}\right)=\chi(A)-s(C) .
$$

Consequentemente,

$$
\chi\left(M_{1}^{+}\right)=\chi\left(M_{0}^{+}\right)-s(C) .
$$

Observamos também que

$$
\chi\left(B^{\prime} \cup C^{\prime}\right)=\chi(B)+1=\chi(B)+s(C) .
$$

Logo

$$
\chi\left(M_{1}^{-}\right)=\chi\left(M_{0}^{-}\right)+s(C) .
$$

Para $s(C)=-1$ (caso onde a região $\mathbf{A}$ é negativa) temos 


$$
\chi\left(A^{\prime}\right)=\chi(A)+s(C) .
$$

Consequentemente,

$$
\chi\left(M_{1}^{+}\right)=\chi\left(M_{0}^{+}\right)+s(C) .
$$

Observamos também que

$$
\chi\left(B^{\prime} \cup C^{\prime}\right)=\chi(B)+1=\chi(B)-s(C) .
$$

Logo

$$
\chi\left(M_{1}^{-}\right)=\chi\left(M_{0}^{-}\right)-s(C) .
$$

Portanto o resultado segue para o caso da transição do tipo $\left(B^{+}\right)$.

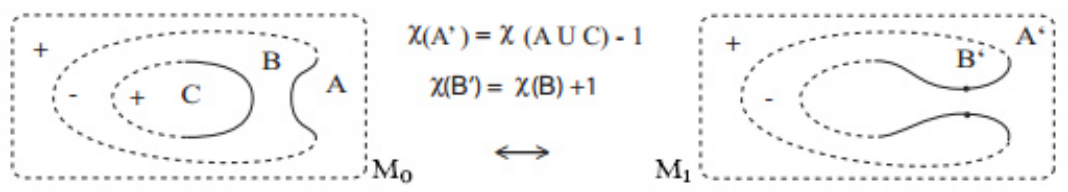

Figura 3.5: [6] Alterando a característica de Euler pela transição $\left(B^{-}\right)$.

Na Figura 3.5, podemos ver uma transição do tipo $\left(B^{-}\right)$. Inicialmente, temos duas componentes de curva de $\Sigma f_{0}$, que separam as regiões $\mathbf{A}, \mathbf{B}$ e $\mathbf{C}$. Após a transição, as duas componentes se unem, de modo que $\Sigma f_{1}$ possui apenas uma componente conexa e com isso, as regiões $\mathbf{A}$ e $\mathbf{C}$ também se unem, dando origem à região $\mathbf{A}^{\prime}$. Neste caso, para as novas regiões $\mathbf{A}^{\prime}$ e $\mathbf{B}^{\prime}$, temos que:

$$
\chi\left(A^{\prime}\right)=\chi(A \cup C)-1=\chi(A)+\chi(C)-1
$$

$\mathrm{e}$

$$
\chi\left(B^{\prime}\right)=\chi(B)+1
$$

Portanto para o caso $s(C)=1$ (caso onde a região A é positiva) temos

$$
\chi\left(M_{1}^{+}\right)=\chi\left(M_{0}^{+}\right)-s(C)
$$




$$
\chi\left(M_{1}^{-}\right)=\chi\left(M_{0}^{-}\right)+s(C)
$$

e para o caso $s(C)=-1$ (caso onde a região $\mathbf{A}$ é negativa) temos

$$
\chi\left(M_{1}^{+}\right)=\chi\left(M_{0}^{+}\right)+s(C)
$$

$\mathrm{e}$

$$
\chi\left(M_{1}^{-}\right)=\chi\left(M_{0}^{-}\right)-s(C)
$$

Portanto, o resultado segue para o caso da transição do tipo $\left(B^{-}\right)$.

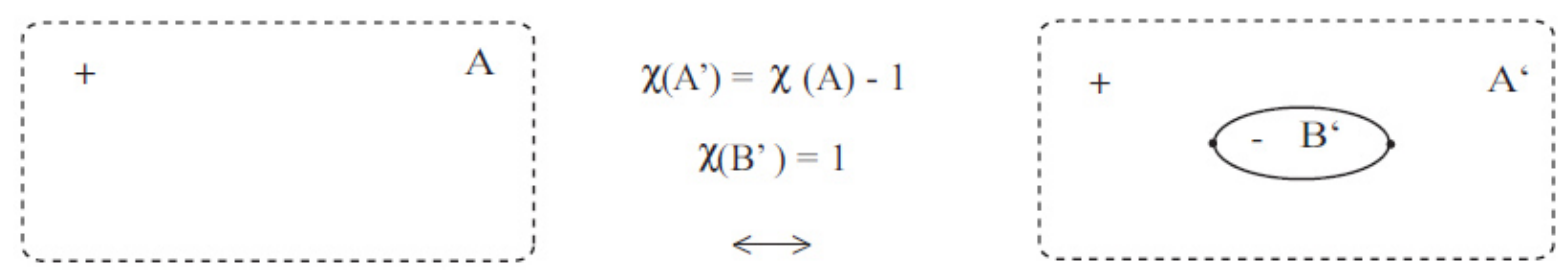

Figura 3.6: [6] Alterando a característica de Euler pela transição $(L)$.

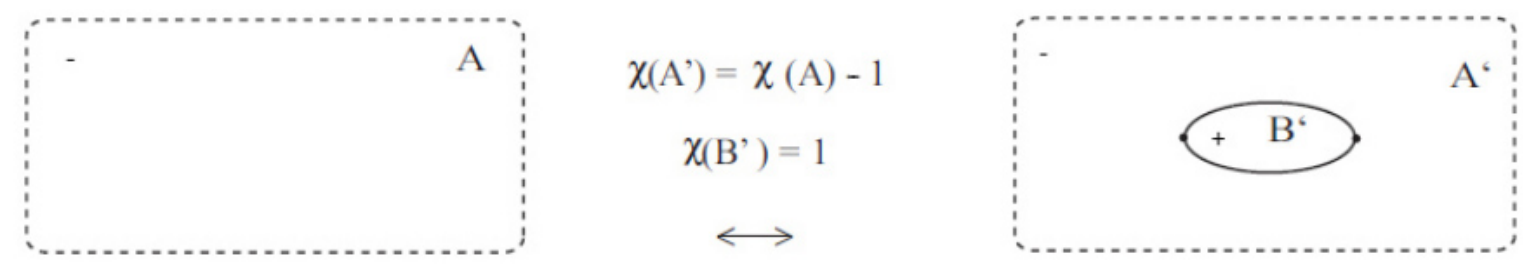

Figura 3.7: [6] Alterando a característica de Euler pela transição $(L)$.

Agora, veremos o caso das transições do tipo lábios (Figuras 3.6 e 3.7). Inicialmente temos uma região A. Depois da transição, temos que a região $\mathbf{A}^{\prime}$ adquire mais uma componente com bordo, portanto a sua característica diminui por um. Além disso, temos o surgimento de uma nova região negativa (positiva), $\mathbf{B}^{\prime}$ homeomorfa a um disco, assim a característica das regiões negativas (positivas) aumenta por um. Como o sinal das cúspides é positivo (negativo), logo o resultado segue.

Observação 3.3.4. A transição do tipo rabo de andorinha não altera a topologia das regiões do complemento do conjunto singular. Logo temos o seguinte resultado: 


$$
\chi\left(M_{1}^{ \pm}\right)=\chi\left(M_{0}^{ \pm}\right) .
$$

Na Figura 3.8, temos uma aplicação estável $f=j \circ g: M \rightarrow S^{2}$, com grau d, onde $M$ é um k-toro, com $k \geq 0, g$ é um mergulho de $M$ deformada em $\mathbb{R}^{3}$ e $j$ é uma projeção da imagem de $g$ na esfera. Usaremos isto na próxima demonstração.
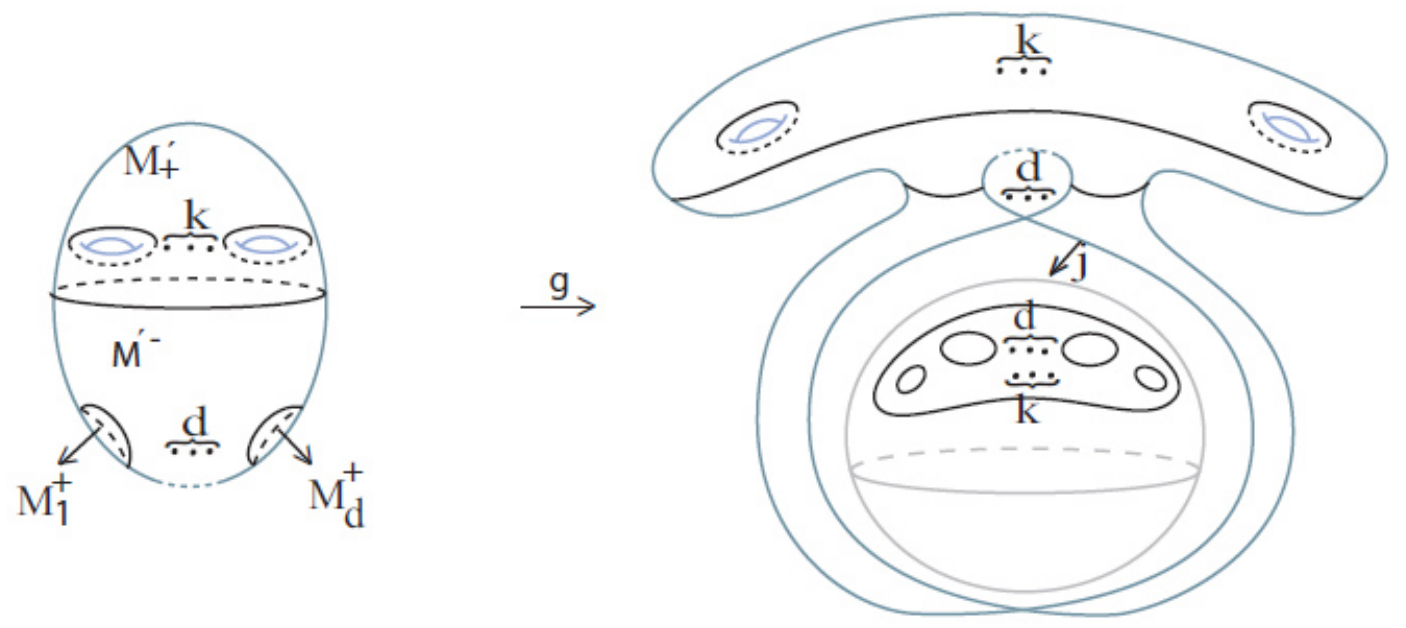

Figura 3.8: [6] Aplicação dobra do k-toro com grau d.

Lema 3.3.5. [6] Seja $f: M \rightarrow S^{2}$ uma aplicação estável de uma superfície $M$ fechada, orientada e de género $k$, na esfera, com grau d, cujo contorno aparente sobre a esfera tenha $d+k+1$ componentes conexas disjuntas e simples. Se $M^{+}$e $M^{-}$são, respectivamente, o fecho das regiões positivas e negativas do complemento do conjunto singular $\Sigma f$, então

$$
\chi\left(M^{+}\right)-\chi\left(M^{-}\right)=2 \operatorname{deg}(f) .
$$

Demonstração: Seja $f: M \rightarrow S^{2}$ uma aplicação estável com grau d, onde a superfície $M$ tem género $\mathrm{k}$ e o conjunto singular tem $\mathrm{d}+\mathrm{k}+1$ componentes sem pontos de cúspides, igual a superfície da Figura 3.8. Então $M^{+}$, o fecho da região de $M$ cuja orientação é preservada por $f$, é decomposto em d +1 componentes, onde d componentes são homeomorfas ao disco, denotadas por $M_{1}^{+}, \ldots, M_{d}^{+}$e a outra componente é homeomorfa ao disco com k buracos, denotada por $M_{+}^{\prime}$, como ilustra a Figura 3.8. Neste caso, a característica de Euler de $M^{+}$é dada por

$$
\chi\left(M^{+}\right)=\chi\left(M_{+}^{\prime}\right)+d=1-k+d .
$$


Por outro lado, temos que, $M^{-}$, o fecho dos pontos regulares de $M$, que tem a orientação invertida por $f$, é uma região homeomorfa a um disco com $\mathrm{k}+\mathrm{d}$ buracos, a qual denotamos por $M_{-}^{\prime}$. Com isso, a característica de Euler de $M^{-}$é dada por

$$
\chi\left(M^{-}\right)=1-k-d .
$$

Logo

$$
\chi\left(M^{+}\right)-\chi\left(M^{-}\right)=2 d .
$$

Teorema 3.3.6. (Corolário do Teorema de Quine [41]). Sejam M uma 2-variedade suave, compacta, orientada e conexa, $f: M \rightarrow S^{2}$ uma aplicação estável, $M^{+}$o fecho do conjunto dos pontos regulares nos quais $f$ preserva a orientação, $M^{-}$o fecho do conjunto dos pontos regulares os quais $f$ inverte a orientação e $C_{1}, \ldots, C_{n}$ pontos de cúspides, então

$$
\chi(M)-2 \chi\left(M^{-}\right)+\Sigma s\left(C_{i}\right)=2 \operatorname{deg}(f),
$$

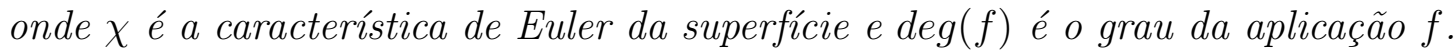

Demonstração: Seja $f_{0}: M_{0} \rightarrow S^{2}$ uma aplicação estável, tal que $\operatorname{deg}\left(f_{0}\right)=\operatorname{deg}(f)$, onde a superfície $M_{0}$ tem género k e o número de componentes singulares é $\operatorname{deg}\left(f_{0}\right)+k+1$, sem cúspides, como ilustra a Figura 3.8. Podemos obter a aplicação $f$ a partir de $f_{0}$, por meio de transições do tipo bicos, lábios ou rabo de andorinha. Vamos denotar por $f_{j}, j \in \mathbb{N}$, a aplicação obtida de $f_{0}$ após $j$ transições do tipo (B), (L) ou (S), e por $M_{i}^{+}\left(M_{i}^{-}\right)$a união das regiões positivas (negativas) do complemento $M \backslash \Sigma f_{i}$. Chamaremos por $C_{j 1}$ e $C_{j 2}$ as cúspides criadas na j-ésima transição. Se a primeira transição for do tipo (L) ou (B), pelo Lema 3.3.3, temos

$$
\begin{gathered}
\chi\left(M_{1}^{+}\right)=\chi\left(M_{0}^{+}\right)-s\left(C_{11}\right), \mathrm{e} \\
\chi\left(M_{1}^{-}\right)=\chi\left(M_{0}^{-}\right)+s\left(C_{12}\right) .
\end{gathered}
$$

Subtraindo as equações acima e usando o Lema 3.3.5, temos

$$
\begin{aligned}
\chi\left(M_{1}^{+}\right)-\chi\left(M_{1}^{-}\right) & =\chi\left(M_{0}^{+}\right)-\chi\left(M_{0}^{-}\right) \chi\left(M_{0}^{-}\right)-\Sigma_{i=1}^{2} s\left(C_{1 i}\right) \\
& =2 \operatorname{deg}\left(f_{0}\right)-\Sigma_{i=1}^{2} s\left(C_{1 i}\right) .
\end{aligned}
$$

Se a primeira transição for do tipo (S), então pela Observação 3.3.4, temos 


$$
\begin{gathered}
\chi\left(M_{1}^{+}\right)=\chi\left(M_{0}^{+}\right) \\
\chi\left(M_{1}^{-}\right)=\chi\left(M_{0}^{-}\right) .
\end{gathered}
$$

Subtraindo as equações acima e utilizando o Lema 3.3.5, temos

$$
\begin{aligned}
\chi\left(M_{1}^{+}\right)-\chi\left(M_{1}^{-}\right) & =\chi\left(M_{0}^{+}\right)-\chi\left(M_{0}^{-}\right) \\
& =2 \operatorname{deg}(f), .
\end{aligned}
$$

mas as cúspides obtidas pelas transições do tipo $(\mathrm{S})$ possuem sinais opostos, com isso $\Sigma_{i=1}^{2} s\left(C_{1 i}\right)=0$, e daí, podemos dizer que

$$
\chi\left(M_{1}^{+}\right)-\chi\left(M_{1}^{-}\right)=2 \operatorname{deg}(f)-\Sigma_{i=1}^{2} s\left(C_{1 i}\right) .
$$

Se a primeira transição for do tipo $(\mathrm{L}),(\mathrm{B})$ ou $(\mathrm{S})$, vale a relação:

$$
\chi\left(M_{1}^{+}\right)-\chi\left(M_{1}^{-}\right)=2 \operatorname{deg}(f)-\Sigma_{i=1}^{2} s\left(C_{1 i}\right) .
$$

Se a segunda transição for do tipo (L) ou (B), pelo Lema 3.3.3, temos

$$
\begin{gathered}
\chi\left(M_{1}^{+}\right)=\chi\left(M_{0}^{+}\right)-s\left(C_{21}\right), \mathrm{e} \\
\chi\left(M_{1}^{-}\right)=\chi\left(M_{0}^{-}\right)+s\left(C_{22}\right) .
\end{gathered}
$$

Subtraindo as equações acima e substituindo a equação 3.1, temos

$$
\begin{aligned}
\chi\left(M_{2}^{+}\right)-\chi\left(M_{2}^{-}\right) & =\chi\left(M_{1}^{+}\right)-\chi\left(M_{1}^{-}\right)-\Sigma_{i=1}^{2} s\left(C_{2 i}\right) \\
& =2 \operatorname{deg}(f)-\Sigma_{i=1}^{2} s\left(C_{1 i}\right)-\Sigma_{i=1}^{2} s\left(C_{2 i}\right) \\
& =2 \operatorname{deg}(f)-\Sigma_{j=1}^{2} \Sigma_{i=1}^{2} s\left(C_{j i}\right) .
\end{aligned}
$$

Se a segunda transição for do tipo (S), então pela Observação 3.3.4, temos

$$
\begin{gathered}
\chi\left(M_{2}^{+}\right)=\chi\left(M_{1}^{+}\right) \\
\chi\left(M_{2}^{-}\right)=\chi\left(M_{1}^{-}\right) .
\end{gathered}
$$

Subtraindo as equações acima e substituindo a equação 3.1, temos

$$
\chi\left(M_{2}^{+}\right)-\chi\left(M_{2}^{-}\right)=2 \operatorname{deg}(f)
$$


mas as cúspides obtidas pelas transições do tipo (S) possuem sinais opostos, $\operatorname{logo} \Sigma_{i=1}^{2} s\left(C_{1 i}\right)=$ 0, e daí, podemos dizer que

$$
\begin{aligned}
\chi\left(M_{2}^{+}\right)-\chi\left(M_{2}^{-}\right) & =2 \operatorname{deg}(f)-\Sigma_{i=1}^{2} s\left(C_{1 i}\right)-\Sigma_{i=1}^{2} s\left(C_{2 i}\right) . \\
& =2 \operatorname{deg}(f)-\Sigma_{j=1}^{2} \Sigma_{i=1}^{2} s\left(C_{j i}\right) .
\end{aligned}
$$

Com isso, vemos que se a segunda transição for do tipo (L), (B) ou (S), vale a relação:

$$
\chi\left(M_{2}^{+}\right)-\chi\left(M_{2}^{-}\right)=2 \operatorname{deg}(f)-\Sigma_{j=1}^{2} \Sigma_{i=1}^{2} s\left(C_{j i}\right) .
$$

Vamos usar indução sobre o número k de transições do tipo (B), (L) ou (S). Suponhamos que para $\mathrm{p}<\mathrm{k}$, vale a relação

$$
\chi\left(M_{2}^{+}\right)-\chi\left(M_{2}^{-}\right)=2 \operatorname{deg}(f)-\Sigma_{j=p}^{2} \Sigma_{i=1}^{2} s\left(C_{j i}\right)
$$

Se a k-ésima transição for do tipo (B), (L) ou (S), pelo Lema 3.3.3 e pela Observação 3.3.4, podemos dizer que

$$
\chi\left(M_{k}^{+}\right)-\chi\left(M_{k}^{-}\right)=\chi\left(M_{k-1}^{+}\right)-\chi\left(M_{k-1}^{-}\right)-\sum_{i=1}^{2} s\left(C_{k i}\right) .
$$

pois as cúspides obtidas pela transição do tipo (S) possuem sinais opostos.

Aplicando a hipótese de indução, temos

$$
\begin{aligned}
\chi\left(M_{k}^{+}\right)-\chi\left(M_{k}^{-}\right) & =2 \operatorname{deg}(f)-\Sigma_{j=1}^{k-1} \Sigma_{i=1}^{2} s\left(C_{j i}\right)-\Sigma_{i=1}^{2} s\left(C_{k i}\right) . \\
& =2 \operatorname{deg}(f)-\sum_{j=1}^{k} \Sigma_{i=1}^{2} s\left(C_{j i}\right) .
\end{aligned}
$$

Daí, obtemos

$$
\chi\left(M^{+}\right)-\chi\left(M^{-}\right)=2 \operatorname{deg}(f)-\Sigma_{i} s\left(C_{i}\right),
$$

ou seja,

$$
\chi\left(M^{+}\right)-\chi\left(M^{-}\right)+\Sigma_{i} s\left(C_{i}\right)=2 \operatorname{deg}(f),
$$

onde $\Sigma_{i} s\left(C_{i}\right)=\sum_{j=1}^{k} \Sigma_{i=1}^{2} s\left(C_{j i}\right)$ e $M^{ \pm}=M_{k}^{ \pm}$. 
Observe que $M^{+} \cap M^{-}$é um conjunto de círculos fechados e portanto $\chi\left(M^{+} \cap M^{-}\right)$ $=0, \log 0 \chi(M)=\chi\left(M^{+}\right)+\chi\left(M^{-}\right)$e voltando a fórmula do Teorema 3.3.6 reescrevemos o teorema da seguinte forma:

Corolário 3.3.7. Com as mesmas hipóteses do Teorema 3.3.6, temos:

$$
\chi\left(M^{+}\right)-\chi\left(M^{-}\right)+\Sigma_{i} s\left(C_{i}\right)=2 \operatorname{deg}(f) .
$$

\subsection{Outras dissertações sobre resultados relacionados ao que estudamos}

Nas dissertações [6], [18] e [33] são estudados os invariantes locais que apresentamos nesse capítulo. Esses invariantes ajudam na classificação das aplicações estáveis.

Vimos seis dissertações que associam grafos à aplicações estáveis [6], [12], [18], [28], [33] e [44]. A seguir, falaremos um pouco de cada uma. Cada dissertação contém as referências dos respectivos artigos estudados, por exemplo [9, 13, 14, 24, 25, 30].

Em [33] é estudado os grafos associados à aplicações estáveis $f: M \rightarrow \mathbb{R}^{2}$, e é provado que todo grafo bipartido pode ser realizado por uma aplicação estável. É visto que uma condição necessária para que um grafo seja realizável por uma aplicação dobra é que ele satisfaça a relação $\left(V^{+}-V^{-}\right)=\left(g^{+}-g^{-}\right)$, onde $V^{+}$é o número de vértices positivos, $V^{-}$é o numero de vértices negativos, $g^{+}$é o género do fecho da região positiva e $g^{-}$é o género do fecho da região negativa. Também é visto uma condição suficiente.

Em [12] é estudado os grafos associados à aplicações estáveis $f: M \rightarrow \mathbb{R}^{2}$, e os grafos associados à aplicações estáveis de 3 -variedades em $\mathbb{R}^{3}$.

Em [6], é estudado os grafos associados à aplicações estáveis $f: M \rightarrow S^{2}$, e é provado que todo grafo bipartido pode ser realizado por uma aplicação estável. É visto também, que todo grafo bipartido $\mathcal{G}$ pode ser realizado por uma aplicação dobra, onde o grau da aplicação dobra é dada por $\mathrm{d}=\left(V^{+}-V^{-}\right)-\left(W^{+}-W^{-}\right)$, a superfície M tem género $1-V+A+W, V^{+}$é o número de vértices positivos, $V^{-}$é o numero de vértices negativos, $W^{+}$é a soma dos pesos dos vértices positivos, $W^{-}$é a soma dos pesos dos vértices negativos, $V$ é o número de vértices, $A$ é o número de arestas e $W$ é a soma do peso de 


\subsection{OUTRAS DISSERTAÇÕES SOBRE RESULTADOS RELACIONADOS AO QUE ESTUDAMOS71}

todos os vértices. Nesse trabalho também é estudado a realização do contorno aparente minimal para aplicações de superfícies orientadas na esfera.

Em [44] é estudado as singularidades da função altura e da aplicação de Gauss. Associa-se os grafos às aplicações de Gauss estáveis, e estuda-se a realização dos grafos.

Em [18] é estudado os grafos associados a aplicações estáveis $f: M \rightarrow S^{2}$, e é provado que todo grafo bipartido pode ser realizado por uma aplicação estável. Nesse trabalho também é estudado a realização do contorno aparente minimal para aplicações de superfícies orientadas na esfera, e também para superfícies não orientadas.

Em [28] é estudado os grafos associados às aplicações estáveis de 3-variedades fechadas no $\mathbb{R}^{3}$. Para esse estudo é preciso de conceitos que não foram abordados nas outras dissertações.

O estudo de aplicações dobras foi abordado nas dissertações [6, 18, 33] seguindo as ideias dos artigos $[11,13,54]$. Este estudo não se aplica para o caso de aplicações de Gauss uma vez que temos que o grau da aplicação de Gauss depende do género da superfície, ou seja, $\operatorname{deg}(\mathrm{N})=1-\mathrm{g}(\mathrm{M})$ (ver [23]). Vimos em [11] que a realização do contorno aparente mínimo também depende do grau da aplicação.

Em nosso trabalho, estudamos os conceitos gerais como os invariantes locais, e a associação de um grafo à uma superfície da forma mais genérica possível, para compreender e tentar uma generalização desses conceitos para 3-variedades fechadas no $\mathbb{R}^{4}$. 


\section{Capítulo 4}

\section{Invariantes de aplicações de Gauss estáveis}

Seguindo o trabalho [30], apresentaremos um novo invariante global para aplicações de Gauss estáveis. Esse invariante é a associação de um grafo bipartido às aplicações de Gauss estáveis de uma superfície fechada e orientada mergulhada em $\mathbb{R}^{3}$. Para falarmos deste estudo, apresentaremos uma definição para associar uma aplicação de Gauss estável a um grafo e veremos os efeitos das cirurgias $S^{-}, S^{-+}$e $S^{+}$e das transições do tipo lábios/bicos nos grafos das aplicações de Gauss estáveis. Veremos alguns casos da aplicação do teorema de Quine para termos uma estimativa do número de cúspides destas aplicações de Gauss. Por fim, veremos que a partir das cirurgias e das transições teremos que para qualquer grafo bipartido podemos associar uma superfície mergulhada em $\mathbb{R}^{3}$ onde a aplicação de Gauss é estável e está associada a esse grafo.

Neste capítulo, apresentaremos apenas as transições que afetam as componentes conexas do conjunto singular. Uma vez que o número de cúspides e o número de pontos duplos não estão presentes no grafo, as transições que interessam são as transições do tipo $A_{3}$, bicos (B) e lábios (L).

As Figuras deste capítulo foram tiradas do trabalho [44]. 


\subsection{Grafos de aplicações de Gauss estáveis}

Definição 4.1.1. Seja $\mathcal{N}: M \rightarrow S^{2}$ uma aplicação de Gauss estável, onde $M$ é uma superfície fechada e orientada e $\Sigma \mathcal{N}$ o conjunto singular de $\mathcal{N}$, que é formado por um conjunto de curvas fechadas. Associamos um grafo $G$, com pesos (inteiros não-negativos) nos vértices, à aplicação $\mathcal{N}$ do seguinte modo:

1. Cada vértice $v_{i}$ corresponde a uma componente conexa do complemento $M \backslash \Sigma \mathcal{N}$ sendo representado por vermelho se a região possui curvatura Gaussiana positiva e por azul se a região possui curvatura Gaussiana negativa. Cada aresta $a_{i}$ corresponde a uma curva $\gamma_{i}$ de $\Sigma \mathcal{N}$;

2. Um vértice $v_{i}$ e uma aresta $a_{i}$ são incidentes se, e somente se, a curva representada por $a_{i}$ encontra-se no bordo da região representada por $v_{i}$;

3. O peso do vértice $v_{i}$ corresponde ao género $g_{i}$ da região correspondente a $M_{i}$, onde $g_{i}=g\left(M_{i}\right)$ é o género da superfície fechada com bordo $M_{i}$.

Como M é orientável, temos que as curvas singulares são curvas fechadas que separam regiões positivas de regiões negativas, logo o grafo $\mathcal{G}_{\mathcal{N}}$ associado à aplicação $\mathcal{N}$ é bipartido.

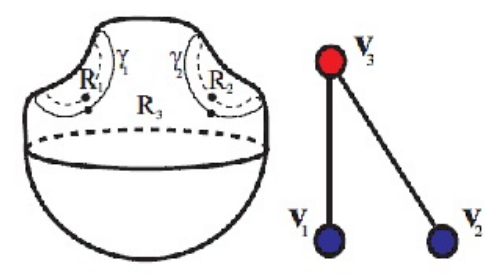

Figura 4.1: Superfície com conjunto parabólico $\left(\gamma_{1} \cup \gamma_{2}\right)$ e o grafo associado

Na superfície acima temos que as curvas $\gamma_{1}$ e $\gamma_{2}$ representam o conjunto parabólico da superfície (que é o conjunto singular da aplicação de Gauss $\mathcal{N}$ ) e os pontos destacados nas curvas representam pontos de cúspides de Gauss.

Temos duas curvas parabólicas $\left(\gamma_{1}\right.$ e $\left.\gamma_{2}\right)$ que separam três regiões distintas $\left(\mathcal{R}_{1}, \mathcal{R}_{2}\right.$ e $\mathcal{R}_{3}$ ). Logo da Definição 4.1.1 temos que cada região $\mathcal{R}_{i}$ é associada a um vértice $v_{i}(i=1,2,3)$. Como a curva $\gamma_{1}$ separa as regiões $\mathcal{R}_{1}$ e $\mathcal{R}_{3}$, temos que os vértices $v_{1} \mathrm{e}$ 
$v_{3}$ têm uma aresta que os liga, da mesma forma temos que $\gamma_{2}$ separa as regiões $\mathcal{R}_{2}$ e $\mathcal{R}_{3}$, $\operatorname{logo}$ temos que os vértices $v_{2}$ e $v_{3}$ têm uma aresta que os liga. $\mathrm{O}$ peso de cada vértice é zero, pois nenhuma região tem género diferente de zero.

Definição 4.1.2. O grau de um vértice $v$ de um grafo é o número de arestas que se conectam a esse vértice.

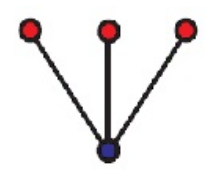

Figura 4.2: Grafos com 3 arestas

Na Figura 4.2 vemos um grafo que possui um vértice negativo com grau 3 e todos os vértices positivos possuem grau 1 .

\subsection{Transições da aplicação de Gauss}

No Capítulo 2, relacionamos algumas singularidades das aplicações de Gauss com singularidades da família de funções altura (Lema 2.2.2). Vimos que para que algumas delas sejam estáveis precisamos estudá-las em famílias de superfícies a 1-parâmetro. Essas famílias de superfícies a 1-parâmetro pode ser vista como transições. Vimos também as seguintes transições:

1. Transições de Morse da curva parabólica em um ponto $A_{3}$, correspondendo a transições do tipo Lábios e bicos na aplicação de Gauss (Figura 2.7).

2. Nascimento/eliminação de um par de cúspides de Gauss em uma curva parabólica (em um ponto $A_{4}$ da função altura). Isto corresponde a uma singularidade do tipo Rabo de Andorinha na aplicação de Gauss (Figura 2.14).

Para a realização dos grafos estamos interessados apenas nas transições que afetam as componentes conexas do conjunto singular de uma superfície, ou seja, as quatro transições do tipo $A_{3}$ : bicos $\left(B^{ \pm}\right)$e lábios $\left(L^{ \pm}\right)$. Estas são as transições que afetam os grafos de uma aplicação de Gauss estável. 


\subsubsection{Grafo da transição lábios}

As transições do tipo lábios $\left(L^{ \pm}\right)$tem como efeito o surgimento ou desaparecimento de um vértice extremo do grafo junto de uma aresta. Uma vez que essa transição ocorre como ilustrado na Figura 2.10 (i) e (ii), temos também o aparecimento ou o desaparecimento de duas cúspides, onde o sinal das cúspides depende da região onde a transição está ocorrendo.

Na Figura 4.3, temos o exemplo da realização da transição lábios em uma região positiva para que surja uma região negativa. Como consequência, no grafo, observamos o surgimento de um vértice negativo e uma aresta ligando esse vértice ao vértice positivo inicial.

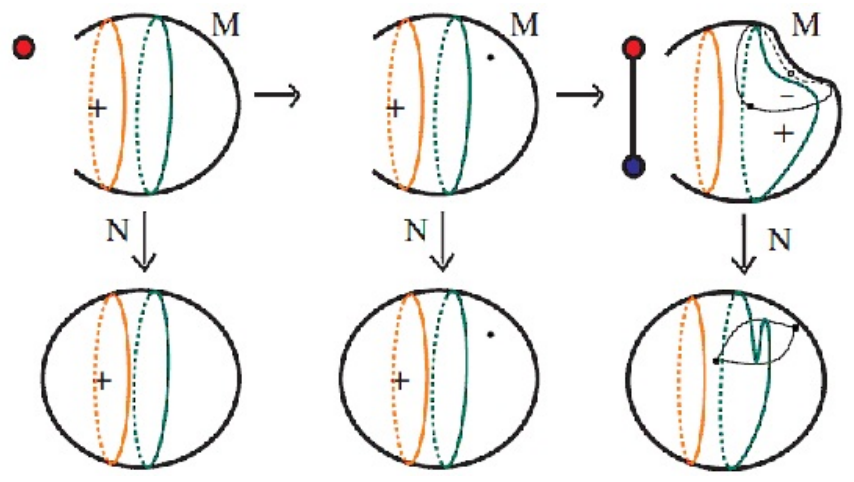

Figura 4.3: Transição (L) e seu grafo local

\subsubsection{Grafo da transição bicos}

As transições do tipo bicos $\left(B^{ \pm}\right)$podem ser usada para unir ou dividir regiões de mesmo sinal de curvatura. Para isso, temos duas formas de realizar a transição do tipo bicos, a primeira é por um processo de tangência das curvas parabólicas em pontos de cúspide que resulta no desaparecimento de duas cúspides de mesmo sinal, a outra forma é por um processo de tangência das curvas parabólicas em pontos de dobra que resulta no aparecimento de duas cúspides de mesmo sinal. Esse processo está ilustrado na Figura 2.7 (iii) e (iv). O efeito desse processo no grafo depende da situação onde ocorre. Na próxima subseção, veremos o que ocorre na Figura 4.1, que é a esfera depois da realização 
de duas transições do tipo lábios em regiões positivas. O segundo caso que veremos, é a realização da transição bicos no toro retorcido (apresentado na Figura 4.7) que nos fornece os toros $T_{1}^{+}$e $T_{1}^{-}$.

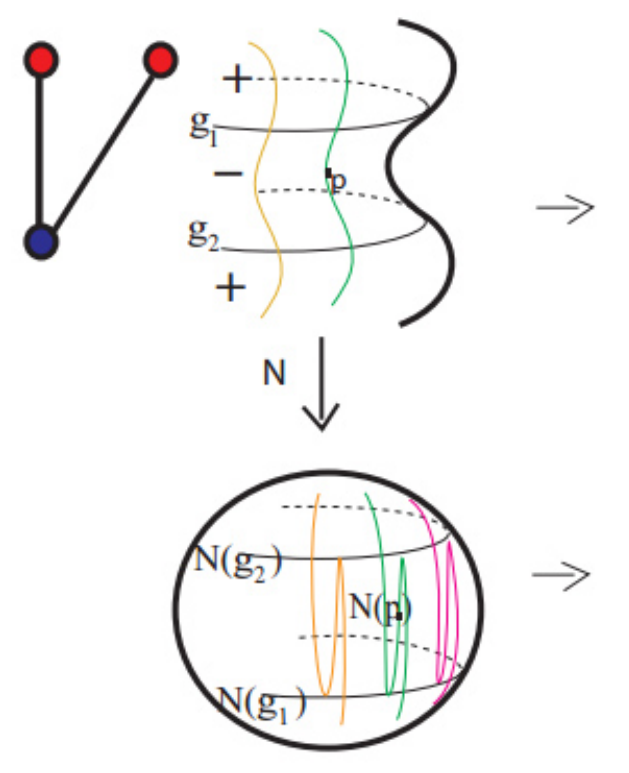

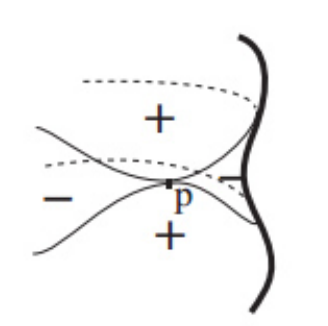
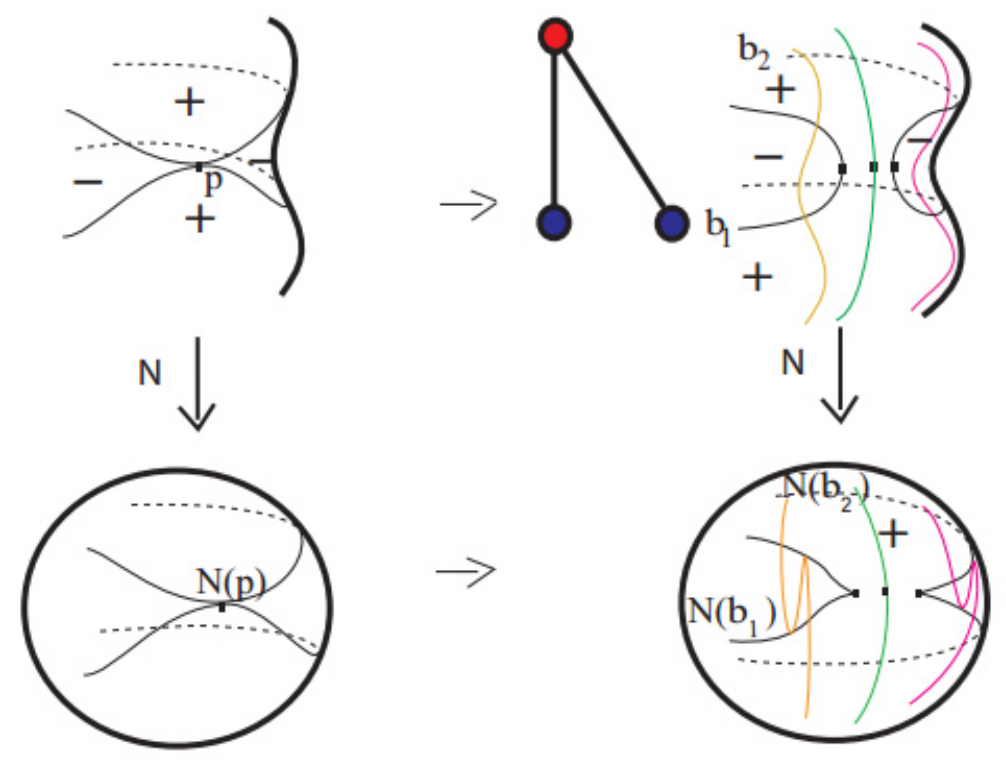
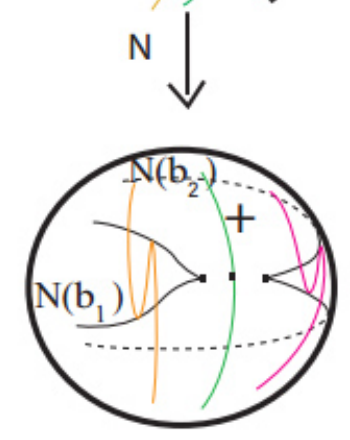

Figura 4.4: Transição (B) e seu grafo local

\subsubsection{Exemplos: realização de grafos}

Esta seção é dedicada à realização de alguns grafos, a partir da esfera e do toro retorcido, utilizando apenas as transições lábios/bicos.

$\mathrm{Na}$ figura a seguir vemos todos os grafos que possuem uma ou duas arestas.
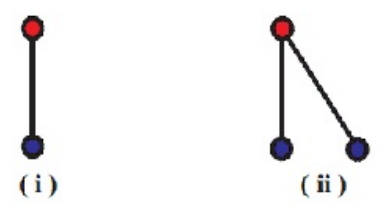

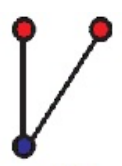

(iii)

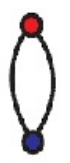

(iv)

Figura 4.5: Grafos básicos

Já vimos a realização dos grafos (i), (ii) e (iii) anteriormente. Na Figura 4.3 temos a realização do grafo (i) e na Figura 4.4 temos realizações dos grafos (ii) e (iii). Na Figura 4.6, vemos realizações desses três grafos a partir da esfera. 


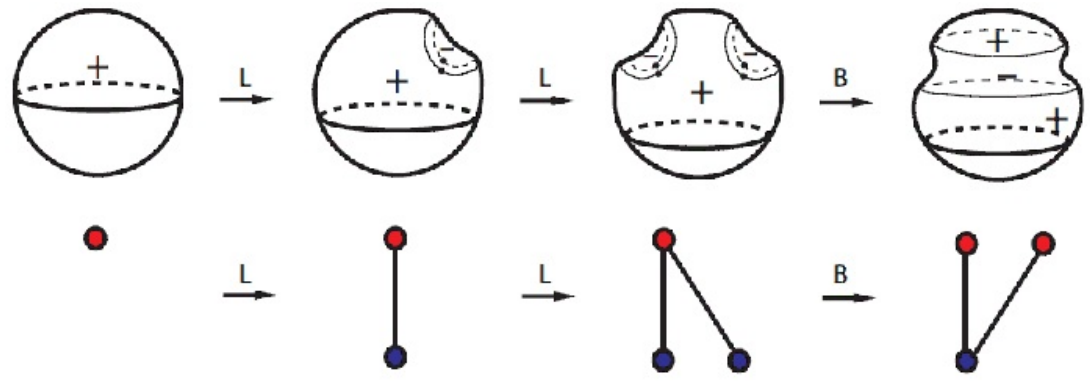

Figura 4.6: Realização dos grafos (i), (ii) e (iii) usando transições

Observe que inicialmente na parte esquerda da figura temos uma esfera e um vértice positivo como seu grafo. Fazendo uma transição (L) obtemos uma superfície $M_{1}$ que realiza (i), fazendo uma nova transição (L) em uma região positiva de $M_{1}$ obtemos uma superfície $M_{2}$ que realiza (ii). Agora, como cada componente do conjunto singular de $M_{2}$ possui duas cúspides de mesmo sinal, pode-se realizar duas transições (B), assim elimina-se as cúspides e obtém-se uma superfície $M_{3}$ que realiza (iii).

Considere a superfície parametrizada por $X(u, v)=\alpha(u)+r(P(u) \cos (v)+B(u) \sin (v))$; onde $\alpha$ é a curva espacial $\alpha(u)=(\cos (u), \sin (u), \epsilon \sin (2 u))$ com P e B o normal principal e o binormal, respectivamente, da curva $\alpha$. Essa superfície é chamada de toro retorcido (ver [4]), seu conjunto singular são duas curvas parabólicas e em cada curva parabólica temos duas cúspides positivas e duas negativas.

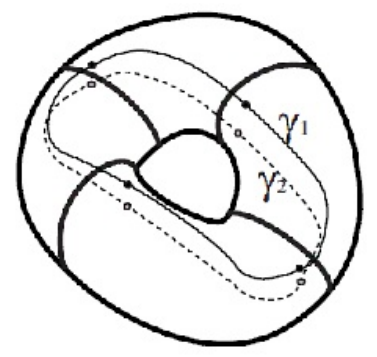

Figura 4.7: Toro Retorcido

Na Figura 4.7 temos uma região positiva e uma região negativa com género zero, e duas curvas parabólicas separando essas regiões, logo temos o grafo (iv) da Figura 4.5 realizado por essa superfície. 
Exemplo 4.2.1. Construiremos utilizando o toro retorcido da Figura 4.7 e a transição (B), superfícies que realizem os grafos da Figura 4.8.

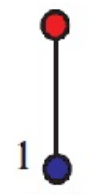

(i)

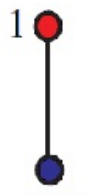

(ii)

Figura 4.8: Grafos com peso diferente de zero

1. Realizamos a transição $B^{-}$em cúspides positivas das curvas parabólicas. Como resultado localmente separamos as regiões positivas e unimos as regiões negativas, como ilustra a Figura 4.9, cuja nova superfície chamamos de $T_{1}^{-}$. Essa nova superfície tem uma região negativa de género 1 e uma positiva de género 0. Assim temos a realização do grafo (i) da Figura 4.8.

2. De forma análoga, realizamos a transição $B^{-}$em cúspides negativas das curvas parabólicas. Como resultado localmente separamos as regiões negativas e unimos as regiões positivas, como ilustra a Figura 4.9, cuja nova superfície chamamos de $T_{1}^{+}$. Essa nova superfície tem uma região negativa de género 0 e uma positiva de género 1. Assim temos a realização do grafo (ii) da Figura 4.8.

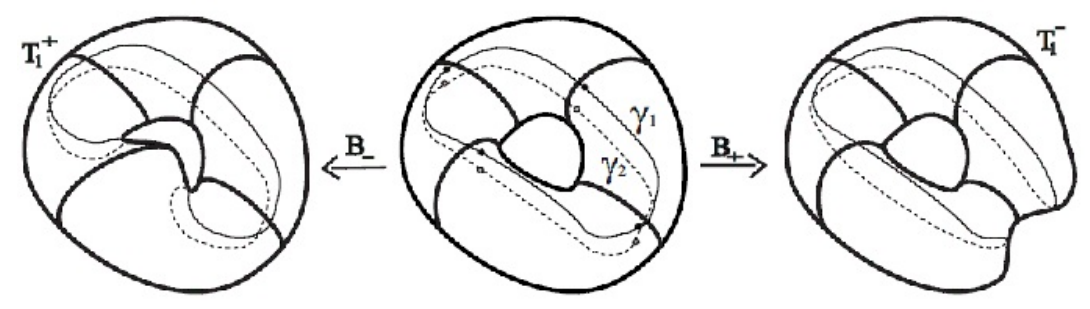

Figura 4.9: $T_{1}^{+}$e $T_{1}^{-}$

Observe que as superfícies obtidas acima possuem ainda 6 cúspides em seu conjunto singular, sendo 4 positivas e 2 negativas em $T_{1}^{+}$e 2 positivas e 4 negativas em $T_{1}^{-}$. 
Observação 4.2.2. Os géneros das regiões positivas ou negativas podem ser aumentados realizando cirurgias $S^{-+}$, que não alteram a estrutura do grafo quando realizado com um dos grafos da Figura 4.8 .

Na Figura 4.10, temos o processo no caso de uma região de curvatura negativa, onde os círculos na cor cinza que aparecem na figura são os círculos que inicialmente retiramos para realizar a cirurgia $S^{-+}$.

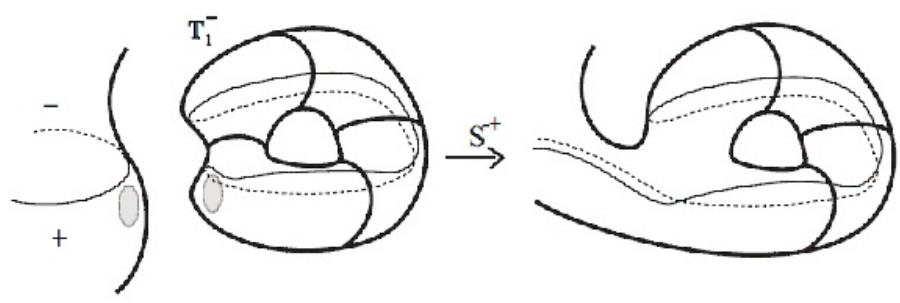

Figura 4.10: Usando a cirurgia $S^{-+}$para aumentar o género de uma região

\subsection{Efeitos das cirurgias nos grafos das aplicações de Gauss de superfícies}

De agora em diante, denotaremos por $\mathrm{A}(\mathrm{M})$ e $\mathrm{V}(\mathrm{M})$ o número de arestas e o número de vértices do grafo associado à aplicação de Gauss da superfície M, respectivamente. Em alguns momentos poderemos utilizar o grafo $\mathcal{G}$ para representar a superfície, e se não existir confusão poderemos usar apenas A e V.

\subsubsection{Grafo da cirurgia $S^{-}$}

A cirurgia $S^{-}$liga dois vértices positivos do mesmo grafo ou de grafos distintos através da criação de uma região hiperbólica, um tubo hiperbólico. Logo com essa cirurgia criamos duas arestas e um vértice negativo.

$\mathrm{Na}$ figura a seguir, vemos o que ocorre localmente no grafo quando realizamos uma cirurgia $S^{-}$.

O resultado a seguir nos mostra o que acontece com o número de arestas, vértices e com o género da superfície. 


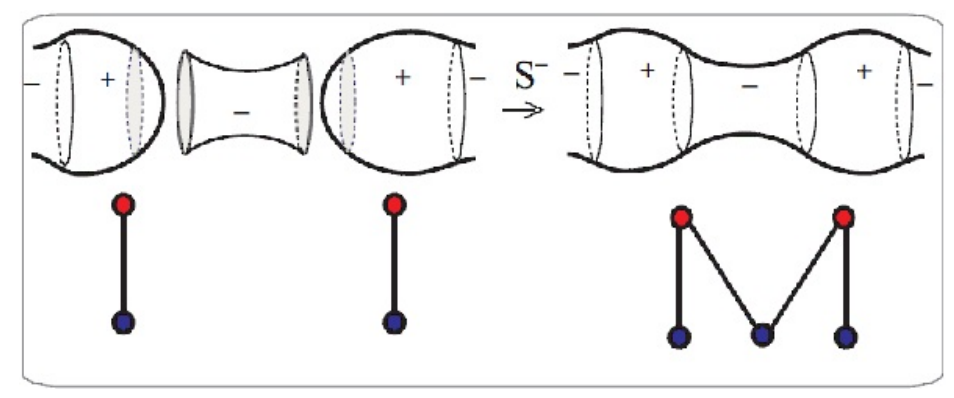

Figura 4.11: Efeito de $S^{-}$no grafo

\section{Proposição 4.3.1. [44]}

1. Sejam $M_{1}$ e $M_{2}$ superfícies distintas e $\mathcal{R}_{1}$ e $\mathcal{R}_{2}$ regiões de curvatura positiva em $M_{1}$ e $M_{2}$, respectivamente. Considere também $M=M_{1} \oplus^{-} M_{2}$ o resultado da cirurgia $S^{-}$em $\mathcal{R}_{1}$ e $\mathcal{R}_{2}$, então $A(M)=A\left(M_{1}\right)+A\left(M_{2}\right)+2, V(M)=V\left(M_{1}\right)+V\left(M_{2}\right)+1$ e $g(M)=g\left(M_{1}\right)+g\left(M_{2}\right)$.

2. Se esta cirurgia é realizada entre duas regiões positivas de uma mesma superfície $M_{0}$ obtendo uma nova superfície $M$, então $A(M)=A\left(M_{0}\right)+2, V(M)=V\left(M_{0}\right)+1$ e $g(M)=g\left(M_{0}\right)+1$.

Demonstração: Primeiro, sejam $M_{1}$ e $M_{2}$ superfícies distintas e $\mathcal{R}_{1}$ e $\mathcal{R}_{2}$ regiões de curvatura positiva em $M_{1}$ e $M_{2}$, respectivamente. Seja $M$ a superfície obtida com a realização da cirurgia $S^{-}$nas regiões $\mathcal{R}_{1}$ e $\mathcal{R}_{2}$, assim temos que o número de arestas de $M$ é $A(M)=A\left(M_{1}\right)+A\left(M_{2}\right)+2$, uma vez que todas as curvas singulares se mantém e além disso, acrescentamos duas novas componentes ao conjunto singular, as curvas singulares que separam as regiões positivas da nova região negativa. O número de vértices é dado por $V(M)=V\left(M_{1}\right)+V\left(M_{2}\right)+1$, uma vez que mantemos todas as regiões de curvaturas positivas e negativas, e acrescentamos uma nova região de curvatura negativa, o tubo hiperbólico. O género é dado por $g(M)=g\left(M_{1}\right)+g\left(M_{2}\right)$, pois a cirurgia $S^{-}$tem o mesmo efeito de uma soma conexa de superfícies.

Agora para o segundo caso, seja $M_{0}$ a superfície, e $\mathcal{R}_{1}$ e $\mathcal{R}_{2}$ duas regiões positivas dessa superfície, onde $M$ é a superfície obtida depois da realização da cirurgia $S^{-}$nas regiões $\mathcal{R}_{1}$ e $\mathcal{R}_{2}$. Igual no primeiro caso, temos que $A(M)=A\left(M_{0}\right)+2$ e $V(M)=V\left(M_{0}\right)+1$. Já o género é dado por $g(M)=g\left(M_{0}\right)+1$, uma vez que esse processo cria um novo ciclo 
independente no grafo, e o número de ciclo independente tem a seguinte relação com o género da superfície

$$
g(M)=\beta(\mathcal{G})+\Sigma g\left(M_{i}^{+}\right)+\Sigma g\left(M_{j}^{-}\right)
$$

onde $\Sigma g\left(M_{i}^{+}\right)$é a soma dos géneros das regiões positivas, e $\Sigma g\left(M_{i}^{-}\right)$é a soma dos géneros das regiões negativas.

Como os géneros das regiões positivas e negativas são mantidos e o género da região negativa nova é zero, o resultado segue.

A fórmula $g(M)=\beta(\mathcal{G})+\Sigma g\left(M_{i}^{+}\right)+\Sigma g\left(M_{j}^{-}\right)$é obtida a partir da construção de uma superfície que realiza o grafo $\mathcal{G}$, onde $\mathcal{G}$ é o grafo de $M$.

\subsubsection{Grafo da cirurgia $S^{-+}$}

Com essa cirurgia, temos o efeito no grafo de 'colar' as arestas que ligam os vértices envolvidos na cirurgia. Temos também o efeito de 'colar' os respectivos vértices positivos assim como os vértices negativos, como ilustra a figura a seguir.

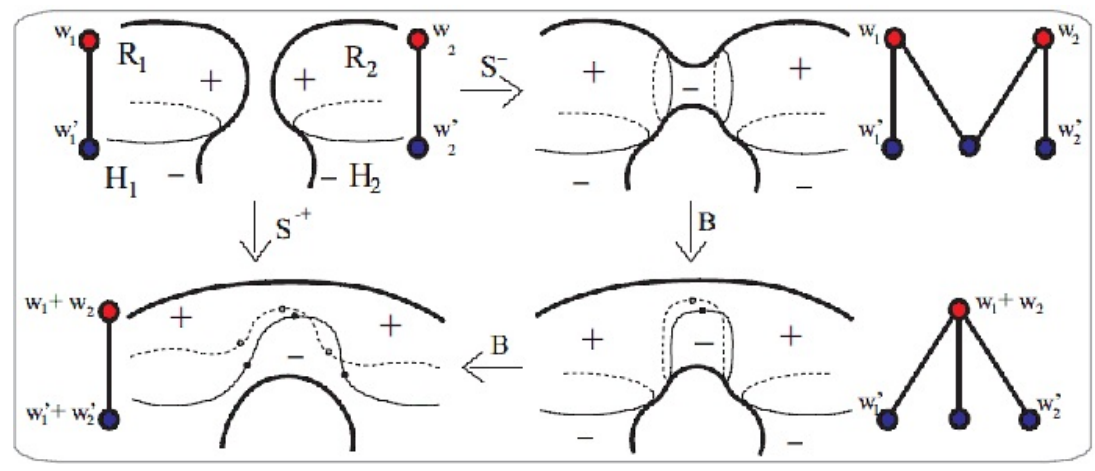

Figura 4.12: Efeito de $S^{-+}$no grafo

\section{Proposição 4.3.2. [44]}

1. Sejam $M_{1}$ e $M_{2}$ superfícies distintas e considere componentes conexas $\mathcal{R}_{1}$ e $\mathcal{H}_{1}$ em $M_{1}$ e $\mathcal{R}_{2}$ e $\mathcal{H}_{2}$ em $M_{2}$, de modo que $\mathcal{R}_{1}$ e $\mathcal{R}_{2}$ são regiões positivas e $\mathcal{H}_{1}$ e $\mathcal{H}_{2}$ são regiões negativas. Além disso, suponha que $\mathcal{R}_{1}$ e $\mathcal{H}_{1}$ possuem a fronteira $\gamma_{1}$ em 
comum e $\mathcal{R}_{2}$ e $\mathcal{H}_{2}$ possuem a fronteira $\gamma_{2}$ em comum. Se realizamos a cirurgia $S^{-+}$ nessas regiões de $M_{1}$ e $M_{2}$ obtendo uma nova superfície $M=M_{1} \oplus^{-+} M_{2}$ então temos os seguintes resultados $A(M)=A\left(M_{1}\right)+A\left(M_{2}\right)-1, V(M)=V\left(M_{1}\right)+$ $V\left(M_{2}\right)-2$ e $g(M)=g\left(M_{1}\right)+g\left(M_{2}\right)$.

2. Se as componentes conexas $\mathcal{R}_{1}, \mathcal{H}_{1}, \mathcal{R}_{2}$ e $\mathcal{H}_{2}$ são distintas e pertencem à uma mesma superfície $M_{0}$ então quando realizamos a cirurgia $S^{-+}$nessas regiões obtemos uma nova superfície $M$ onde $A(M)=A\left(M_{0}\right)-1, V(M)=V\left(M_{0}\right)-2$ e $g(M)=g\left(M_{0}\right)+1$.

A prova desta proposição é analoga à prova da Proposição 4.3.1.

Observação 4.3.3. Da Observação 4.2.2, temos que qualquer grafo com pelo menos uma aresta pode ter qualquer peso de seus vértices aumentado. Para isso, basta realizar cirurgias $S^{-+}$com o toro $T_{1}^{+}$ou $T_{1}^{-}$, dependendo se queremos aumentar o género do vértice positivo ou do vértice negativo, como apresentado no exemplo na Figura 4.10.

\subsubsection{Grafo da cirurgia $S^{+}$}

A cirurgia $S^{+}$'cola' duas regiões positivas do mesmo grafo ou de grafos diferentes, assim temos que o efeito dessa cirurgia é a 'colagem' dos vértices que representam essas regiões.

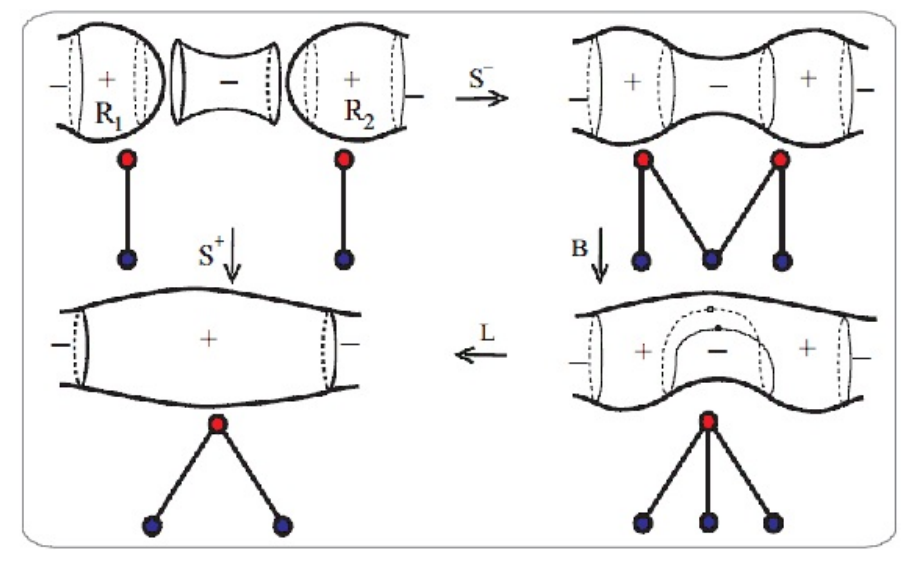

Figura 4.13: Efeito de $S^{+}$no grafo

A proposição a seguir pode ser demonstrada de maneira análoga à demonstração apresentada para a Proposiç̧ão 4.3.1. 
Proposição 4.3.4. [44]

1. Sejam $M_{1}$ e $M_{2}$ superfícies distintas e considere componentes conexas positivas $\mathcal{R}_{1}$ e $\mathcal{R}_{2}$ em $M_{1}$ e $M_{2}$, respectivamente. Se realizamos a cirurgia $S^{+}$em $M_{1}$ e $M_{2}$ obtendo uma nova superfície $M=M 1 \oplus^{+} M 2$ então $A(M)=A\left(M_{1}\right)+A\left(M_{2}\right)$, $V(M)=V\left(M_{1}\right)+V\left(M_{2}\right)-1$ e $g(M)=g\left(M_{1}\right)+g\left(M_{2}\right)$.

2. Se as componentes conexas $\mathcal{R}_{1}$ e $\mathcal{R}_{2}$ pertencem a uma mesma superfície $M_{0}$ e se realizamos a cirurgia $S^{+}$em $M_{0}$, unindo essas regiões, então $A(M)=A\left(M_{0}\right)$, $V(M)=V\left(M_{0}\right)-1$ e $g(M)=g\left(M_{0}\right)+1$.

\subsection{Realização de grafos bipartidos}

A prova da realização de qualquer grafo bipartido com peso, é obtida através de algumas induções. Provaremos,inicialmente, que qualquer árvore sem peso nos vértices, é realizada. Para isso, utilizamos uma indução sobre o número de vértices. Depois provaremos que todos os grafos bipartidos sem peso nos vértices podem ser realizados, e para isso utilizamos uma indução sobre o número de Betti. Finalmente, provaremos a realização de qualquer grafo bipartido e para isso usaremos a Observação 4.3.3.

\subsubsection{Grafos bipartidos com número de arestas $\leq 3$}

Já apresentamos todos os possíveis grafos com uma ou duas arestas na Figura 4.5, e apresentamos superfícies que realizam esses grafos. A seguir, apresentaremos uma proposição que é consequência da Observação 4.3.3.

Proposição 4.4.1. [44] O grafo composto por dois vértices e apenas uma aresta com pesos $k$ (vértice positivo) e l (vértice negativo), apresentado na Figura 4.14, pode ser realizado por uma aplicação de Gauss estável de uma superfície fechada e orientada imersa no $\mathbb{R}^{3}$.

Demonstração: Para $k=l=0$ temos que o grafo é realizado pela superfície obtida na Figura 4.3. Para o caso onde k e l podem ser não nulos, basta realizar k e l cirurgias do tipo $S^{-+}$entre regiões positivas e negativas com o toro $T_{1}^{+}$e $T_{1}^{-}$, respectivamente. 


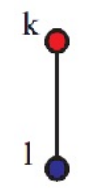

Figura 4.14: Grafo simples com pesos

Nas Figuras 4.6 e 4.7, temos superfícies que realizam todos os grafos com uma ou duas arestas. Para realizar esses grafos com peso arbitrários basta realizar cirurgias do tipo $S^{-+}$com o toro $T_{1}^{+}$e $T_{1}^{-}$. Provando a proposição a seguir.

Proposição 4.4.2. [44] Todo grafo bipartido com pesos e duas arestas pode ser realizado por uma aplicação de Gauss estável de uma superfície fechada e orientada imersa no $\mathbb{R}^{3}$.

A seguir veremos os grafos com 3 arestas.

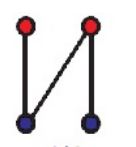

(i)

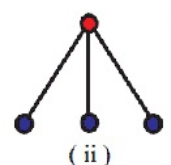

(ii)

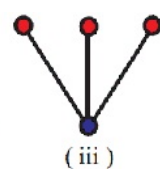

(iii

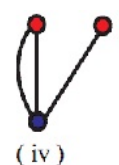

iv)

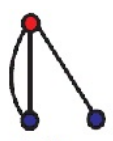

(v)

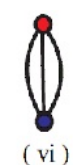

Figura 4.15: Grafos com 3 arestas

Proposição 4.4.3. [44] Os grafos de 3 arestas da Figura 4.15 podem ser realizados a partir da cirurgia $S^{-+}$entre as superfícies que realizam os grafos de 2 arestas (Figura 4.5).

Demonstração: Basta observar o efeito da cirurgia $S^{-+}$nos grafos de 2 arestas, uma vez que já vimos o resultado desta cirurgia nos grafos na Subseção 4.3.2 (Figuras 4.16, 4.17 e 4.18). 


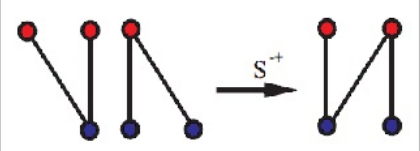

(i)

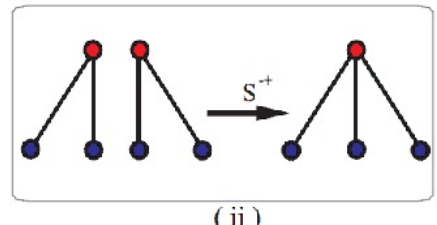

(ii)

Figura 4.16: Realização dos grafos $(i)$ e (ii) da Figura 4.15

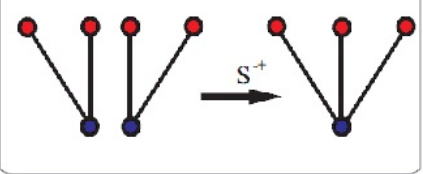

(iii )

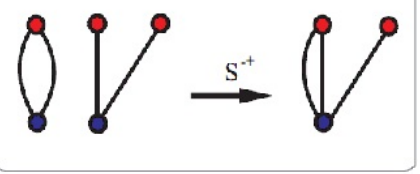

(iv)

Figura 4.17: Realização dos grafos (iii) e (iv) da Figura 4.15

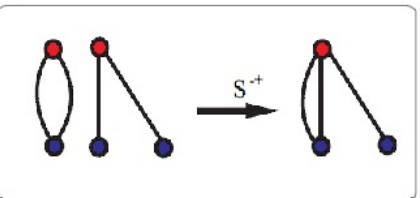

( v )

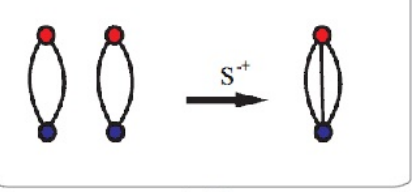

(vi)

Figura 4.18: Realização dos grafos $(v)$ e $(i v)$ da Figura 4.15

\subsubsection{Realização de grafos árvore e grafos bipartidos}

Este primeiro resultado é importante para a demonstração do teorema de grafos bipartidos.

Teorema 4.4.4. [30] Qualquer árvore com peso zero em cada um de seus vértices e pelo menos uma aresta pode ser realizada por uma aplicação de Gauss estável de uma imersão da esfera, $f: S^{2} \rightarrow \mathbb{R}^{3}$.

Demonstração: Faremos a prova por indução. Vemos que a árvore com peso zero em cada um de seus vértices e com uma aresta que está representada na Figura 4.5 (i), é realizada por uma superfície obtida após uma transição do tipo lábios na esfera, como ilustra as duas superfícies na Figura 4.6.

Assumindo que toda árvore com peso zero em cada um de seus vértices e com $n$ arestas, é realizada por uma aplicação de Gauss estável de uma imersão da esfera, $h: S^{2} \rightarrow \mathbb{R}^{3}$, vamos provar que uma árvore com peso zero em cada um de seus vértices e com $n+1$ 
arestas é realizada.

Seja $\mathcal{T}$ uma árvore com $n+1$ arestas, e seja $v$ um vértice extremo de $\mathcal{T}$, ou seja, $v$ é um vértice de grau 1 . Considere a árvore $\mathcal{T}^{\prime}$ como sendo a árvore $\mathcal{T}$ sem o vértice $v$ e sem a única aresta $w$ que liga $v$ a um outro vértice $u$, ou seja $\mathcal{T}^{\prime}$ é uma árvore com $n$ arestas. Logo, $\mathcal{T}^{\prime}$ é realizada por alguma imersão $g: S^{2} \rightarrow \mathbb{R}^{3}$ cuja aplicação de Gauss possui $\mathcal{T}^{\prime}$ como grafo. Agora realizando uma transição do tipo lábios na região que é representada por $u$, obtemos uma imersão $f: S^{2} \rightarrow \mathbb{R}^{3}$ que realiza a árvore $\mathcal{T}$, pois a nova aresta e o novo vértice são os que retiramos para ter a árvore $\mathcal{T}^{\prime}$.

Teorema 4.4.5. [30] Qualquer grafo bipartido o qual todos os vértices possuem peso zero pode ser realizado por uma aplicação de Gauss estável de uma superfície fechada orientável $M$, com $\chi(M)=2-2 \beta(\mathcal{G})$, onde $\beta(\mathcal{G})$ é o número de Betti de $\mathcal{G}$ (isto é, o número de ciclos independentes no grafo).

Demonstração: Provaremos, esse teorema, por indução. O Teorema 4.4.4 nos dá o caso onde o número de ciclos independentes é zero, e a fórmula $\chi(M)=2-2 \beta(\mathcal{G})$ é válida, uma vez que temos que $M$ é um mergulho da esfera em $\mathbb{R}^{3}$ e $\beta(\mathcal{G})=0$, onde $M$ é a superfície que realiza $\mathcal{G}$, e onde $\mathcal{G}$ é a árvore do Teorema 4.4.4.

Suponha que o resultado seja válido quando o grafo possui $n$ ciclos independentes.

Agora provaremos que o resultado é válido para grafos com $n+1$ ciclos independentes. Seja $\mathcal{G}$ um grafo com $n+1$ ciclos independentes. Escolhendo um dos ciclos independentes de $\mathcal{G}$ e escolhendo um vértice positivo $v$ desse ciclo podemos separar esse vértice positivo em dois vértices positivos, $v_{1}$ e $v_{2}$. Obtemos assim, um grafo $\mathcal{G}^{\prime} \operatorname{com} n$ ciclos independentes que é realizado por uma imersão $g: M_{0} \rightarrow \mathbb{R}^{3}$ e $\chi\left(M_{0}\right)=2-2 n$. Realizando uma cirurgia do tipo $S^{+}$nas regiões $\mathcal{R}_{1}$ e $\mathcal{R}_{2}$ correspondentes aos vértices (positivos) $v_{1}$ e $v_{2}$ de $\mathcal{G}_{0}$, obtemos uma superfície M com $\chi(M)=2-2(n+1)$ e uma imersão $f: M \rightarrow \mathbb{R}^{3}$ cuja aplicação de Gauss possui $\mathcal{G}$ como seu grafo. Além disso, pela Proposição 4.3.4, temos que $g(M)=g\left(M_{0}\right)+1$. Segue que $\chi(M)=2-2 \beta(\mathcal{G})$ onde $\beta(\mathcal{G})=g(M)$, e $g(M)$ é o género da superfície $M$. 
Consideramos agora, grafos bipartidos com pesos para mostrarmos sua realização.

Teorema 4.4.6. [30]. Qualquer grafo bipartido $\mathcal{G}$ com pesos em seus vértices pode ser realizado por uma aplicação de Gauss estável de uma superfície fechada e orientável $M$, com $\chi(M)=2-2(\beta(\mathcal{G})+\omega(\mathcal{G}))$, onde $\beta(\mathcal{G})$ é o número de Betti de $\mathcal{G}$, e $\omega(\mathcal{G})$ é a soma dos pesos em cada vértice de $\mathcal{G}$ (peso total de $\mathcal{G}$ ).

Demonstração: Provaremos esse teorema por indução sobre o peso total do grafo $\mathcal{G}$.

Temos que o grafo é realizado quando o peso total é zero, segundo o Teorema 4.4.5. Considere o resultado válido para grafos com peso total $\leq p$. Seja $\mathcal{G}$ um grafo com peso total $p+1$, e seja $v$ um vértice de $\mathcal{G}$ com peso $w_{1}$ diferente de zero. Considere o grafo $\mathcal{G}_{0}$ igual ao grafo $\mathcal{G}$ a menos do peso do vértice $v$. Em $\mathcal{G}_{0}$ o peso de $v$ é $w_{1}-1$, assim $\mathcal{G}_{0}$ tem peso total igual a p. Logo, pela hipótese de indução temos que o grafo $\mathcal{G}_{0}$ é realizado por uma superfície fechada e orientável $M_{0}$, onde a fórmula é valida, assim $\chi\left(M_{0}\right)=2-2(\beta(\mathcal{G})+p)$. Agora para obter o grafo $\mathcal{G}$ a partir de $\mathcal{G}_{0}$, utilizamos a Observação 4.3.3, assim somamos peso 1 à supefície, e o resultado segue.

Observação 4.4.7. Da fórmula do teorema anterior temos a fórmula

$$
g(M)=\beta(\mathcal{G})+\Sigma g\left(M_{i}^{+}\right)+\Sigma g\left(M_{j}^{-}\right)
$$

De fato, do Teorema 4.4.6 temos que $g(M)=\beta(\mathcal{G})+\omega(\mathcal{G})$, e $\omega(\mathcal{G})=\Sigma g\left(M_{i}^{+}\right)+$ $\Sigma g\left(M_{j}^{-}\right)$, onde $\Sigma g\left(M_{i}^{+}\right)+\Sigma g\left(M_{j}^{-}\right)$é a soma dos pesos das regiões positivas e negativas, respectivamente.

\subsubsection{Cúspides da aplicação de Gauss estável}

Nesta seção, apresentaremos um invariante que é a soma dos sinais das cúspides $\Sigma s\left(C_{i}\right)$ para a aplicação de Gauss estável. Vamos ver algumas relações entre $\Sigma s\left(C_{i}\right)$ e o grafo, e apresentaremos o caso das árvores estreladas onde temos uma aplicação de Gauss que realiza esse tipo de grafo sem nenhuma cúspide. 
Proposição 4.4.8. [30] O número $\mu$ de componentes conexas da curva parabólica de uma superfície fechada e orientável, genericamente imersa em $\mathbb{R}^{3}$, é dado por

$$
\mu=2\left(V^{-}-g^{-}\right)-\frac{1}{2}\left(C^{+}-C^{-}\right)
$$

onde $V^{-}, g^{-}, C^{-}$e $C^{+}$representam respectivamente o número de vértices negativos, soma dos pesos dos vértices negativos, o número de cúspides negativas e o número de cúspides positivas.

Demonstração: Seja $\mathcal{N}$ a aplicação de Gauss da superfície M. Temos pelo Corolário 3.3.7 que $2 \operatorname{deg}(\mathcal{N})=\chi\left(M^{+}\right)-\chi\left(M^{-}\right)+\Sigma_{i} s\left(C_{i}\right)$ onde $\Sigma_{i} s\left(C_{i}\right)$ reescrevemos como $C^{+}-C^{-}$. Em [23], temos que $\operatorname{deg}(\mathcal{N})=1-g(M)$, onde $g(M)$ é o género da superfície M. Mais ainda, pela Observação 4.4.7 temos que $g(M)=g\left(M^{+}\right)+g\left(M^{-}\right)+\beta(\mathcal{G})$ onde $\beta(\mathcal{G})$ é o número de Betti do grafo $\mathcal{G}$ de $\mathcal{N}$ (número de ciclos independentes de $\mathcal{G}$ ).

Portanto, obtemos:

$$
2\left(1-g\left(M^{+}\right)-g\left(M^{-}\right)-\beta(\mathcal{G})\right)=\chi\left(M^{+}\right)-\chi\left(M^{-}\right)+C^{+}-C^{-} .
$$

que nos leva a,

$$
2 \beta(\mathcal{G})=2\left(1-g\left(M^{+}\right)-g\left(M^{-}\right)\right)+\chi\left(M^{-}\right)-\chi\left(M^{+}\right)-C^{+}+C^{-}
$$

Agora como

$$
\chi\left(M_{i}^{-}\right)=2-2 g\left(M_{i}^{-}\right) \text {e } \chi\left(M_{i}^{+}\right)=2-2 g\left(M_{i}^{+}\right)
$$

onde $M_{i}^{-}$é uma componente conexa de $M^{-}$e $M_{i}^{+}$é uma componente conexa de $M^{+}$, segue que

$$
\begin{gathered}
\chi\left(M^{-}\right)=\Sigma \chi\left(M_{i}^{-}\right)=\Sigma\left(2-2 g\left(M_{i}^{-}\right)\right)=2\left(V^{-}-g\left(M^{-}\right)\right), \mathrm{e} \\
\chi\left(M^{+}\right)=\Sigma \chi\left(M_{i}^{+}\right)=\Sigma\left(2-2 g\left(M_{i}^{+}\right)\right)=2\left(V^{+}-g\left(M^{+}\right)\right) .
\end{gathered}
$$

Logo, temos que $\chi\left(M^{-}\right)-\chi\left(M^{+}\right)=2\left(g\left(M^{+}\right)-g\left(M^{-}\right)+V^{-}-V^{+}\right)$e substituindo na fórmula anterior temos que:

$$
2 \beta(\mathcal{G})=2-4 g\left(M^{-}\right)+2 V^{-}-2 V^{+}-C^{+}+C^{-} .
$$


Além disso, pela Definição 1.2.9, $\beta(\mathcal{G})=\mu-V+1$, com $V=V^{+}+V^{-}$, e da igualdade anterior substituindo o valor de $\beta(\mathcal{G})$ temos:

$$
\mu=2\left(V^{-}-g^{-}\right)-\frac{1}{2}\left(C^{+}-C^{-}\right),
$$

onde a soma dos pesos dos vértices negativos representa o género de $M^{-}$, ou seja, $g^{-}$é $g\left(M^{-}\right)$

Observação 4.4.9. Com as hipóteses da proposição anterior, se a aplicação de Gauss de uma imersão genérica não possui cúspides, o número $\mu$ de componentes conexas da curva parabólica é par.

Agora, veremos o número $\Sigma s\left(c_{i}\right)$ para alguns grafos. Começaremos com o grafo (i) da Figura 4.5. Se aplicarmos o Corolário do Teorema de Quine 3.3.7 à superfície que realiza esse grafo temos o seguinte resultado:

$$
\Sigma_{i=1}^{2} s\left(c_{i}\right)=2 \operatorname{deg} \mathcal{N}-\chi\left(M^{+}\right)+\chi\left(M^{-}\right),
$$

o que nos dá:

$$
\sum_{i=1}^{2} s\left(c_{i}\right)=2
$$

que condiz com o fato da transição lábios gerar duas cúspides positivas.

Proposição 4.4.10. [44] O grafo composto por dois vértices e apenas uma aresta, com pesos $k$ (vértice positivo) e l (vértice negativo), apresentado na Figura 4.14, tem a seguinte propriedade

$$
\Sigma s\left(c_{i}\right)=2(1-2 l)
$$

onde $s\left(c_{i}\right)$ é o sinal da cúspide do ponto $c_{i}$.

Demonstração: Por [23], temos que $\operatorname{deg} \mathcal{N}=1-g(M)$, onde $\mathcal{N}$ é a aplicação de Gauss da superfície $M$ e $g(M)$ é o seu género. Além disso, temos que $g(M)=\beta(\mathcal{G})+g\left(M^{+}\right)+g\left(M^{-}\right)$, onde $\mathcal{G}$ é o grafo da aplicação de Gauss de M, e como o grafo de M não possui ciclos temos que $g(M)=g\left(M^{+}\right)+g\left(M^{-}\right)$. 
Como $M$ é uma superfície sem bordo, temos que $\chi(M)=2-2 g(M)$. Os géneros de $M_{i}^{+}$e de $M_{I}^{-}$foram definidos em 4.1.1(3), e a partir deles definimos os géneros de $M^{+} \mathrm{e}$ de $M^{-}$. Assim, $g\left(M^{+}\right)=\Sigma g\left(M_{i}^{+}\right)$e $g\left(M^{-}\right)=\Sigma g\left(M_{i}^{-}\right)$. Como $M^{+}$e $M^{-}$são superfícies com bordo temos que $\chi\left(M^{+}\right)=2-2 g\left(M^{+}\right)-t$ onde t é o número de componentes de bordo da superfície $M^{+}$e $\chi\left(M^{-}\right)=2-2 g\left(M^{-}\right)-t$ onde t é o número de componentes de bordo da superfície $M^{-}$. Note que temos o mesmo $t$ para $\chi\left(M^{+}\right)$e $\chi\left(M^{-}\right)$uma vez que esse $t$ representa o conjunto parabólico que é a fronteira das regiões de $\chi\left(M^{+}\right)$e de $\chi\left(M^{-}\right)$ao mesmo tempo.

Das observações acima, e do Corolário 3.3.7 que nos dá a equação, $\Sigma s\left(c_{i}\right)=2 \operatorname{deg} \mathcal{N}$ $\chi\left(M^{+}\right)+\chi\left(M^{-}\right)$temos:

$$
\begin{aligned}
\Sigma s\left(c_{i}\right) & =2(1-g(M))-(2-2 k-t)+(2-2 l-t) \\
& =2\left(1-g\left(M^{+}\right)-g\left(M^{-}\right)\right)+2(k-l) \\
& =2(1-k-l)+2(k-l) \\
& =2(1-2 l)
\end{aligned}
$$

Na proposição acima vemos que $\Sigma s\left(C_{i}\right)$, o somatório das cúspides da superfície $M$, só depende do peso do vértice negativo.

Note que para realizar o grafo da Figura 4.14 podemos realizar por cirurgias do tipo $S^{-+}$, que soma quatro cúspides negativas e duas positivas. As cirurgias são realizadas com as superfícies $T_{1}^{+}$e $T_{1}^{-}$que foram obtidas na Figura 4.9, e que possuem o somatório das cúspides igual a 2 e -2 respectivamente. Assim, temos que quando realizamos uma cirurgia $S^{-+}$entre uma superfície $\mathrm{M}$ e $T_{1}^{+}$não alteramos a somatória das cúspides, e quando realizamos uma cirurgia $S^{-+}$entre uma superfície M e $T_{1}^{-}$temos assim quatro cúspides negativas que alteram o somatório das cúspides da superfície $M$.

Com essa fórmula, concluímos que o grafo (i) da Figura 4.5 não pode ser realizado sem cúspides, pois caso este fato fosse verdadeiro teríamos $0=\Sigma s\left(C_{i}\right)=2(1-2 l)$, o que nos dá $l=\frac{1}{2}$, que é absurdo.

A seguir, definimos um tipo de grafo especial onde podemos encontrar o número mínimo de cúspide. 
Definição 4.4.11. Uma árvore estrelada de grau n positiva (negativa) é uma árvore que possui apenas um vértice positivo (negativo) com grau $n$, onde $n \geq 2$.

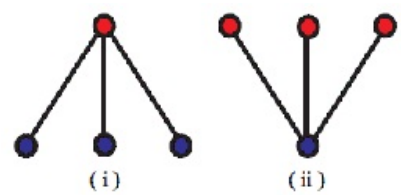

Figura 4.19: Árvores estreladas

A Figura 4.20 possui exemplos de árvores estreladas de grau 3, sendo $(i)$ uma árvore positiva e (ii) uma árvore negativa.

Proposição 4.4.12. [44] Toda árvore estrelada positiva de grau n e peso zero em seus vértices é realizável por uma aplicação de Gauss estável com

$$
\Sigma s\left(C_{i}\right)=2 n
$$

Além disso, o minimo de cúspides é $2 n$ cúspides positivas.

Demonstração: Sabemos que esta árvore pode ser realizada devido ao Teorema 4.4.4.

Sabendo que $M^{+}$possui apenas uma componente conexa e $M^{-}$possui n componentes conexas temos que $\chi\left(M^{+}\right)=2$ e $\chi\left(M^{-}\right)=2 n$. Aplicamos agora o Teorema 3.6, observando que $g(M)=0$, e obtemos:

$$
\begin{aligned}
\Sigma s\left(c_{i}\right) & =2 \operatorname{deg}(\mathcal{N})-2+2 n \\
& =2(1-g(M))-2+2 n \\
& =2 n .
\end{aligned}
$$

Sabemos que em cada transição (L) "nascem" duas cúspides, sendo duas positivas se a transição é realizada numa região de curvatura positiva e duas negativas se a transição é realizada numa região de curvatura negativa.

Portanto, podemos realizar um grafo estrelado positivo de grau n realizando n transições (L) distintas em $S^{2}$, assim teremos 0 cúspides negativas e 2 n cúspides positivas. 
Proposição 4.4.13. [44] Toda árvore estrelada negativa de grau $n$ e peso zero em seus vértices é realizável por uma aplicação de Gauss estável com

$$
\Sigma s\left(C_{i}\right)=2(2-n) .
$$

Além disso, o mínimo de cúspides é $2(2-n)$ cúspides negativas.

Demonstração: A prova é análoga à prova da Proposição 4.4.12. Realizamos o grafo estrelado negativo de grau n fazendo a seguinte construção: seja o grafo (iii) da Figura 4.5 (uma árvore estrelada negativa de grau 2), onde a aplicação de Gauss é do tipo dobra, ou seja, sem cúspide. Tomando a superfície da Figura 4.6 que realiza esse grafo, para obter um grafo estrelado negativo de grau $n$ basta fazer $n-2$ transições (L) na região de curvatura negativa que nos dará 0 cúspides positivas e $2(n-2)$ cúspides negativas.

O caso de árvore estrelada positiva de grau 5 pode ser realizado com 5 transições (L) na esfera $S^{2}$, obtendo a Figura 4.21 .

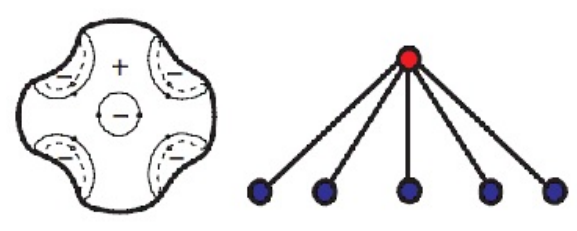

Figura 4.20: Árvore estrelada positiva de grau 5

Apresentaremos duas formas de realizar a árvore estrelada negativa de grau 5( ver Figuras 4.22 e 4.23). A primeira forma é realizada fazendo três transições (L) no grafo (iii) da Figura 4.6. A segunda forma é realizada à partir da sequência de transições apresentada na Figura 4.24, onde 2(L) e 2(B) representam a realização de duas transições (L) e duas transições (B), respectivamente.

Com esse exemplo, temos duas superfícies que possuem o mesmo grafo, mas não tem aplicações de Gauss $\mathcal{A}$-equivalentes, uma vez que uma possui 2 curvas singulares sem cúspide e a outra possui 3 curvas singulares sem cúspide.

Agora, veremos um resultado para grafos bipartidos. 


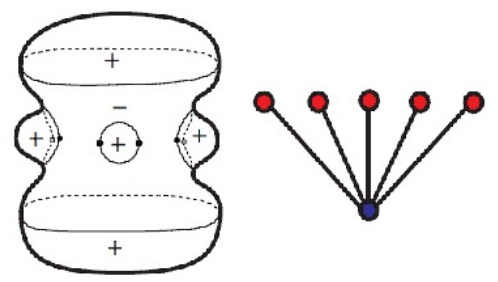

Figura 4.21: $1^{\circ}$ Caso: Árvore estrelada negativa de grau 5

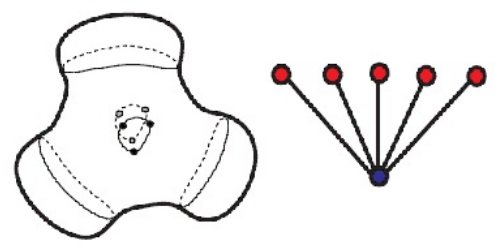

Figura 4.22: $2^{\circ}$ Caso: Árvore estrelada negativa de grau 5

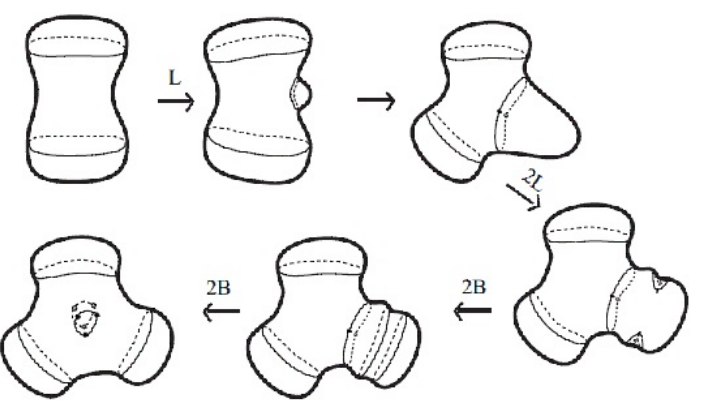

Figura 4.23: Transições para obter a árvore estrelada negativa de grau 5 da Figura 4.23

Teorema 4.4.14. [44] Para um grafo bipartido $\mathcal{G}$ que realiza uma aplicação de Gauss temos que

$$
\Sigma s\left(c_{i}\right)=2\left(A-V-2 \omega\left(V^{-}\right)\right),
$$

onde $A$ é o número de arestas, $V=V^{+}+V^{-}$é o número de vértices e $\omega\left(V^{-}\right)$é a soma dos pesos de $V^{-}$para o grafo $\mathcal{G}$.

Demonstração: Pelo Corolário, 3.3.7 temos $\Sigma s\left(c_{i}\right)=2 \operatorname{deg}(\mathcal{N})-\chi\left(M^{+}\right)+\chi\left(M^{-}\right)$e além disso, $\operatorname{deg} \mathcal{N}=1-g(M)$ onde $g(M)=g\left(M^{+}\right)+g\left(M^{-}\right)+\beta(\mathcal{G})$. Logo,

$$
\Sigma s\left(c_{i}\right)=2-4 g\left(M^{-}\right)+2 \beta(\mathcal{G})
$$

Pela Definição 1.2.9, temos que $\beta(\mathcal{G})=1-V+A$, e além disso $g\left(M^{-}\right)=\omega\left(V^{-}\right)$, de onde o resultado segue fazendo estas substituições. 
Corolário 4.4.15. Uma condição necessária para que a aplicação de Gauss não possua cúspides é que o grafo associado a ela satisfaça a seguinte equação

$$
A-V=2 \omega\left(V^{-}\right)
$$

Temos que a condição dada no corolário não é suficiente para que a aplicação de Gauss seja uma aplicação dobra, pois o grafo do Toro Retorcido (grafo (iv) da Figura 4.5) satisfaz a equação mas seu conjunto singular possui 8 cúspides, sendo 4 positivas e 4 negativas.

\subsection{Algoritmo para a realização dos grafos}

A partir das provas por indução dos Teomemas 4.4.4, 4.4.5 e 4.4.6 temos um método recorrente para construir uma superfície que realize um grafo bipartido dado.

Uma vez que a Observação 4.3.3 nos dá uma forma de aumentar os pesos dos vértices do grafo bipartido, só precisamos de uma forma de construir uma superfície que realiza um grafo com peso zero e pelo menos uma aresta.

Estamos querendo uma forma de realizar qualquer grafo bipartido com pelo menos uma aresta, isso porque o grafo com apenas um vértice positivo é realizado pela esfera, e o grafo com apenas um vértice negativo não é realizável pois não existe superfície hiperbólica compacta sem bordo mergulhado em $\mathbb{R}^{3}$.

Sempre é possível considerar um grafo $\mathcal{T}$ (Definição 1.2.11) que é a árvore geradora do grafo $\mathcal{G}$ que queremos realizar.

Para um grafo bipartido $\mathcal{G}$ com pelo menos uma aresta seguimos os seguintes passos:

1. Consideramos o grafo bipartido $\mathcal{G}$, porém com peso igual a zero em todos os seus vértices;

2. Encontramos uma árvore geradora $\mathcal{T}$ de $\mathcal{G}$;

Realizamos a árvore geradora $\mathcal{T}$ de $\mathcal{G}$ da seguinte forma:

3. Temos que $\mathcal{G}$ possui pelo menos uma aresta, assim também acontece com $\mathcal{T}$, portanto podemos escolher um vértice positivo $v$ de $\mathcal{T}$. Construiremos uma superfície que 
realiza a árvore $\mathcal{T}$ a partir da esfera $S^{2}$ que realiza o grafo bipartido com um único vértice positivo.

4. Para cada vértice $w_{i}$ de $\mathcal{T}$, que possui uma aresta ligando $w_{i}$ a $v$ realizamos uma transição (L) em $S^{2}$, obtendo uma nova superfície, com $i$ variando de 1 a $n-1$, onde o grafo possui $n$ vértices.

5.i Agora, se o vértice $w_{i}$ na árvore geradora $\mathcal{T}$ é um vértice extremo, não fazemos nada na região que este representa. Caso contrário, realizamos uma transição lábios na região $w_{i}$ para cada vértice $w_{j_{1}}, \ldots, w_{j_{k}}$ que está ligado à $w_{i}$, exceto os vértices $\mathrm{v}$ e $w_{i}$ dos passos anteriores, onde os índices $j_{1}, \ldots, j_{k}$ são distintos dos índices usados nos passos anteriores.

Agora, veremos duas formas de construir os ciclos, que são duas formas de ligar um vértice positivo $v$ a um negativo $w$. A primeira forma de construir essa ligação é usando a cirurgia $S^{-+}$. Para usar essa cirurgia precisamos realizar uma transição (L) nas regiões que representam os vértices $v$ e $w$, e depois basta realizar a cirurgia. A segunda forma de construir essa ligação é usando a cirurgia $S^{+}$. Para usar essa cirurgia precisamos realizar uma transição (L) na região negativa, e depois realizamos a cirurgia $S^{+}$entre a nova região positiva e a região que representa o vértice $v$. Cada método resulta em superfícies diferentes visto que o número de cúspides obtidas em cada caso é diferente.

6. Para realizar o grafo $\mathcal{G}$ basta construir os ciclos em $\mathcal{T}$ usando um dos métodos descritos acima.

Assim, realizamos o grafo $\mathcal{G}$ sem pesos em seus vértices.

7. Para realizarmos qualquer grafo $\mathcal{G}$ com peso, basta usarmos a Observação 4.3.3.

\subsection{Matrizes de aplicação de Gauss estáveis}

A seguir, definiremos classes de matrizes para representarem os grafos bipartidos com peso. Definiremos as equivalências $M_{\mathcal{G}}, M_{\mathcal{G}, c}$ e $M_{\mathcal{G}, c, d}$. Estas definições são ideias que 
surgiram durante este trabalho de dissertação. Daremos exemplos de superfícies que não são $\mathcal{A}$-equivalentes e cujas matrizes, de seus grafos, estão na mesma classe $M_{\mathcal{G}}$. Para estas superfícies as classes $M_{\mathcal{G}, c}$ das superfícies as distinguem. As classes $M_{\mathcal{G}, c, d}$ são mais completas por conterem informações sobre mais invariantes.

Esta ideia surgiu por causa das matrizes de incidência e adjacência(Definições 1.2.3 e 1.2.4) da teoria de grafos (ver [31]).

Definição 4.6.1. Seja $\mathcal{G}$ um grafo bipartido com peso, onde $\left\{v_{i}^{+}\right\}$são os n vértices positivos $e\left\{v_{i}^{-}\right\}$são os $m$ vértices negativos. Definimos a matriz $\left(x_{i j}\right), 1 \leq i \leq n+1$, $1 \leq j \leq m+1$, onde

- $x_{i j}$ é igual ao número de arestas ligando o vértice $v_{i}^{+}$ao vértice $v_{j}^{-}$para $1 \leq i \leq n$ $e 1 \leq j \leq m$,

- $x_{i j}=\omega\left(v_{i}^{+}\right)$para $1 \leq i \leq n$ e $j=m+1$,

- $x_{i j}=\omega\left(v_{j}^{-}\right)$para $1 \leq j \leq m$ e $i=n+1$,

- $x_{i j}=0$ para $i=n+1$ e $j=m+1$.

Essa é a matriz representante do grafo com pesos.

A seguir, temos dois grafos isomorfos com suas respectivas matrizes associadas.

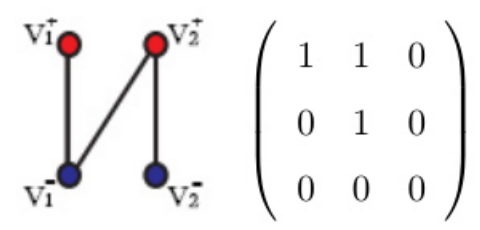

Figura 4.24: Grafo 1 e sua matriz

Esse exemplo mostra que se renomearmos os vértices de $\mathcal{G}$ podemos obter uma matriz nova que representa o mesmo grafo. Para termos uma unicidade de representantes podemos considerar classes de equivalência.

Definição 4.6.2. Duas matrizes $m$ por $n$ são ditas $M_{\mathcal{G}}$-equivalentes se conseguirmos obter uma matriz da outra realizando um número finito de operaçôes do tipo troca de linha, entre 
Figura 4.25: Grafo 2 e sua matriz

as linhas $\{1, \ldots, m-1\}$, e um número finito de operações do tipo troca de coluna, entre as colunas $\{1, \ldots, n-1\}$.

Pela definição anterior, temos uma associação dos grafos com as classes de matrizes $M_{\mathcal{G}}$. Para cada grafo bipartido podemos obter uma matriz representante de uma $M_{\mathcal{G}}$ classe de equivalência, o contrário nem sempre é válido. A matriz, a seguir é um exemplo de uma classe que não possui um grafo para representá-la. Lembre que estamos considerando que os grafos são conexos.

$$
\left(\begin{array}{lll}
1 & 0 & 0 \\
0 & 1 & 0 \\
0 & 0 & 0
\end{array}\right)
$$

Definição 4.6.3. Seja $\mathcal{G}$ um grafo bipartido com peso, associado à aplicação de Gauss da imersão $f: M \rightarrow \mathbb{R}^{3}$, onde $\left\{v_{i}^{+}\right\}$são os $n$ vértices positivos e $\left\{v_{i}^{-}\right\}$são os $m$ vértices negativos, $\left\{a_{k}\right\}$ são as arestas. Definimos a matriz $\left(x_{i j}\right), 1 \leq i \leq n+1+k$ e $1 \leq j \leq$ $m+1+k$ onde

- $x_{i j}$ é igual ao número de arestas ligando o vértice $v_{i}^{+}$ao vértice $v_{j}^{-}$para $1 \leq i \leq n$ $e 1 \leq j \leq m$,

- $x_{i j}=\omega\left(v_{i}^{+}\right)$para $1 \leq i \leq n$ e $j=m+1$,

- $x_{i j}=\omega\left(v_{j}^{-}\right)$para $1 \leq j \leq m$ e $i=n+1$,

- $x_{i j}=0$ para $i \geq n+1$ e $j=m+1$,

- $x_{i j}=0$ para $i=n+1$ e $j \geq m+1$,

- $x_{i j}$ é igual ao número de cúspides apontadas para a direção da região representada por $v_{j}^{-}$presente na curva singular representada por $a_{i-n-1}$, ou $x_{i j}=-1$ caso a 
região representada por $v_{j}^{-}$não tenha como fronteira a curva singular representada por $a_{i-n-1}$, para $i>n+1$ e $j<m+1$,

- $x_{i j}$ é igual ao número de cúspides apontadas para a direção da região representada por $v_{i}^{+}$presente na curva singular representada por $a_{j-m-1}$, ou $x_{i j}=-1$ caso a região representada por $v_{i}^{+}$não tenha como fronteira a curva singular representada por $a_{j-m-1}$, para $i<n+1$ e $j>m+1$,

- $x_{i j}=0$ para $i>n+1$ e $j>m+1$,

Essa é a matriz representante da aplicação de Gauss da superfície, com informações sobre o género das regióes conexas do complemento do conjunto singular e informações sobre as cúspides.

Definimos uma outra classe de equivalência, similar a vista acima, da seguinte forma.

Definição 4.6.4. Duas matrizes $m+1+k$ por $n+1+k$ são ditas $M_{\mathcal{G}, c}$-equivalentes se conseguirmos obter uma matriz da outra realizando um número finito de operações do tipo troca de linhas, entre as linhas $\{1, \ldots, m\}$, um número finito de operações do tipo troca de coluna, entre as colunas $\{1, \ldots, n\}$, e um número finito de operações do tipo linha/coluna, entre as linhas $\{n+2, \ldots, n+1+k\}$ se para cada uma dessas operações realizarmos uma operação similar nas colunas $\{m+2, \ldots, m+1+k\}$, isto é se trocarmos a linha $n+i+l$ pela linha $n+1+p$ trocaremos a coluna $m+1+$ l pela coluna $n+1+p$.

A seguir, temos duas superfícies com suas classes $M_{\mathcal{G}, c}$, respectivamente. Observamos que essas classes são distintas, portanto as classes $M_{\mathcal{G}, c}$ servem para diferenciar as superfícies das Figuras 4.27 e 4.28. Estas superfícies possuem os mesmos grafos, logo as mesmas classes $M_{\mathcal{G}}$, mas não são $\mathcal{A}$-equivalentes como já vimos na Subseção 4.4.3. 

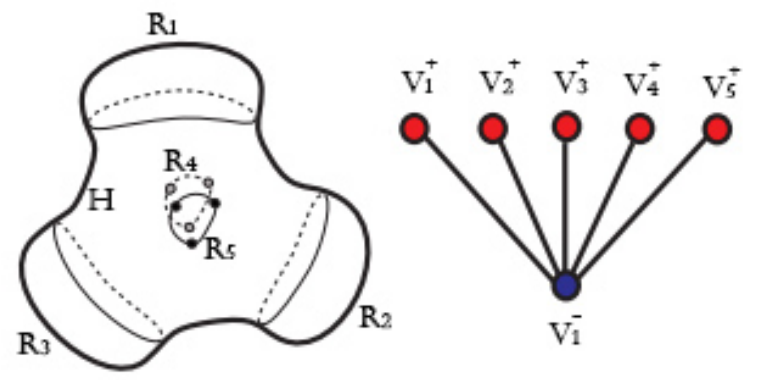

Figura 4.26: Grafo 1

$$
\left(\begin{array}{ccccccc}
1 & 0 & 0 & -1 & -1 & -1 & -1 \\
1 & 0 & -1 & 0 & -1 & -1 & -1 \\
1 & 0 & -1 & -1 & 0 & -1 & -1 \\
1 & 0 & -1 & -1 & -1 & 0 & -1 \\
1 & 0 & -1 & -1 & -1 & -1 & 0 \\
0 & 0 & 0 & 0 & 0 & 0 & 0 \\
0 & 0 & 0 & 0 & 0 & 0 & 0 \\
0 & 0 & 0 & 0 & 0 & 0 & 0 \\
0 & 0 & 0 & 0 & 0 & 0 & 0 \\
3 & 0 & 0 & 0 & 0 & 0 & 0 \\
3 & 0 & 0 & 0 & 0 & 0 & 0
\end{array}\right)
$$

Essa matriz é um representante da classe da matriz $M_{\mathcal{G}, c}$ da aplicação de Gauss da superfície da Figura 4.27. 


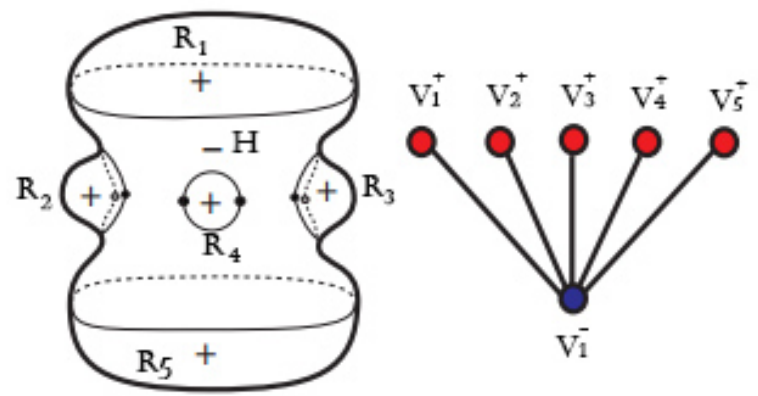

Figura 4.27: Grafo 2

$$
\left(\begin{array}{ccccccc}
1 & 0 & 0 & -1 & -1 & -1 & -1 \\
1 & 0 & -1 & 0 & -1 & -1 & -1 \\
1 & 0 & -1 & -1 & 0 & -1 & -1 \\
1 & 0 & -1 & -1 & -1 & 0 & -1 \\
1 & 0 & -1 & -1 & -1 & -1 & 0 \\
0 & 0 & 0 & 0 & 0 & 0 & 0 \\
0 & 0 & 0 & 0 & 0 & 0 & 0 \\
2 & 0 & 0 & 0 & 0 & 0 & 0 \\
2 & 0 & 0 & 0 & 0 & 0 & 0 \\
2 & 0 & 0 & 0 & 0 & 0 & 0 \\
0 & 0 & 0 & 0 & 0 & 0 & 0
\end{array}\right)
$$

Essa matriz é um representante da classe da matriz $M_{\mathcal{G}, c}$ da aplicação de Gauss da superfície da Figura 4.28.

Definição 4.6.5. Seja $\mathcal{G}$ um grafo bipartido com peso, associado à aplicação de Gauss da imersão $f: M \rightarrow \mathbb{R}^{3}$, onde $\left\{v_{i}^{+}\right\}$são os $n$ vértices positivos e $\left\{v_{i}^{-}\right\}$são os $m$ vértices negativos, $\left\{a_{k}\right\}$ são as arestas. Definimos a matriz $\left(x_{i j}\right), 1 \leq i \leq n+1+k$ e $1 \leq j \leq$ $m+1+k$ onde

- $x_{i j}$ é igual ao número de arestas ligando o vértice $v_{i}^{+}$ao vértice $v_{j}^{-}$para $1 \leq i \leq n$ $e 1 \leq j \leq m$,

- $x_{i j}=\omega\left(v_{i}^{+}\right)$para $1 \leq i \leq n$ e $j=m+1$, 
- $x_{i j}=\omega\left(v_{j}^{-}\right) \operatorname{para} 1 \leq j \leq m$ e $i=n+1$,

- $x_{i j}=0$ para $i \geq n+1$ e $j=m+1$.

- $x_{i j}=0$ para $i=n+1$ e $j \geq m+1$

- $x_{i j}$ é igual ao número de cúspides apontadas para a direção da região representada por $v_{j}^{-}$presente na curva singular representada por $a_{i-n-1}$, ou $x_{i j}=-1$ caso a região representada por $v_{j}^{-}$não tenha como fronteira a curva singular representada por $a_{i-n-1}$, para $i>n+1$ e $j<m+1$,

- $x_{i j}$ é igual ao número de cúspides apontadas para a direção da região representada por $v_{i}^{+}$presente na curva singular representada por $a_{j-m-1}$, ou $x_{i j}=-1$ caso a região representada por $v_{i}^{+}$não tenha como fronteira a curva singular representada por $a_{j-m-1}$, para $i<n+1$ e $j>m+1$,

- $x_{i j}=0$ para $i>n+1$ e $j>m+1$,

- $x_{i j}$ é igual ao número de pontos duplos entre as curvas singulares representadas por $a_{i-n-1}$ e $a_{j-n-1}$ para $i>n+1$ e $j>m+1$ e $j-n-1 \neq i-n-1$

- $x_{i j}=0$ para $i>n+1$ e $j>m+1$ e $j-n-1=i-n-1$.

Essa é a matriz representante do grafo, com informações sobre as cúspides e os pontos duplos.

Definimos a classe $M_{\mathcal{G}, c, d}$ da mesma forma que a classe $M_{\mathcal{G}, c}$, a diferença está no fato das matrizes das classes $M_{\mathcal{G}, c, d}$ terem os pontos duplos na parte quadrada simétrica $\{n+2, \ldots n+1+k\},\{m+2, \ldots m+1+k\}$.

Definição 4.6.6. Duas matrizes $m+1+k$ por $n+1+k$ são ditas $M_{\mathcal{G}, c, d}$-equivalentes se conseguirmos obter uma matriz da outra realizando um número finito de operações do tipo troca de linhas, entre as linhas $\{1, \ldots, m\}$, um número finito de operações do tipo troca de colunas, entre as colunas $\{1, \ldots, n\}$, e um número finito de operações do tipo linha/coluna, entre as linhas $\{n+2, \ldots, n+1+k\}$, se para cada uma dessas operações realizarmos uma operação similar nas colunas $\{m+2, \ldots, m+1+k\}$, isto é se trocarmos a linha $n+i+l$ pela linha $n+1+p$ trocaremos a coluna $m+1+l$ pela coluna $n+1+p$. 
Temos que ambas as classes $M_{\mathcal{G}}, M_{\mathcal{G}, c}$ e $M_{\mathcal{G}, c, d}$ são invariantes por $\mathcal{A}$-equivalência, uma vez que os grafos, as cúspides, os pontos duplos e o género são invariantes. A classe $M_{\mathcal{G}, c, d}$ é um invariante mais completo porém não trabalhamos com os pontos duplos das aplicações de Gauss, assim ainda não temos nenhum exemplo não trivial de classe $M_{\mathcal{G}, c, d}$ onde o número de pontos duplos é não nulo. Para o caso onde o número de pontos duplos é nulo, temos que a matriz do tipo $M_{\mathcal{G}, c}$ é igual a matriz $M_{\mathcal{G}, c, d}$.

Essas matrizes podem ser associadas à aplicações de uma superfície na esfera, ou de uma superfície no plano, uma vez que todos os elementos usados para definir essas matrizes estão presentes nesses dois casos. 


\section{Conclusão e perspectivas futuras}

O objetivo desse trabalho foi entender o conceito de grafos apresentado em [30] para podermos buscar resultados similares para 3-variedades em $\mathbb{R}^{4}$ no doutorado. O trabalho [30] associa grafos à superfícies compactas sem bordo mergulhadas em $\mathbb{R}^{3}$, que tenham a aplicação de Gauss estável. Estudamos as singularidades das aplicações de Gauss utilizando a família de funções altura e a aplicação catástrofe dessa família. Desta maneira, relacionamos as singularidades da função altura com as singularidades da aplicação de Gauss pois essa forma de analisar a aplicação de Gauss pode ser estendida para dimensões maiores. Por fim, introduzimos classes de matrizes como invariantes.

Em pesquisas futuras poderemos também:

1. Buscar propriedades das classes de matrizes como invariantes, e tentar chegar a um invariante completo,

2. Encontrar invariantes para 3-variedades mergulhadas em $\mathbb{R}^{4}$ seguindo o mesmo raciocinio dos grafos para superfícies mergulhadas em $\mathbb{R}^{3}$. 


\section{Referências Bibliográficas}

[1] Y. Ando. Existence theorems of fold maps. Japan J. Math., 30:29-73, 2004.

[2] V. Arnold. Plane curves, their invariants, perestroikas and classifications. Advances in Soviet Math., (41):33-91, 1994.

[3] V. I. Arnold, S. M. Gusein-Zade, and A. N. Varchenko. Singularities of Differentiable Maps: The classification of critical points, caustics and wave fronts. Monographs in mathematics. Birkhäuser, 1985.

[4] T. Banchoff, T. Gaffney, and C. McCrory. Cusps of gauss mappings. Web version by Daniel Dreibelbis, 1982.

[5] J. Boardman. Singularities of Differentiable Maps. University of Warwick, 1965.

[6] J. Bretas. Singularidades de aplicações estáveis de superfícies fechadas e orientadas em $S^{2}$. Dissertação de mestrado, Universidade Federal de Viçosa, 2011.

[7] J. W. Bruce and P. J. Giblin. Curves and Singularities. Cambridge University Press, 2 edition, 1992.

[8] J. W. Bruce, P. J. Giblin, and F. Tari. Families of surfaces: height functions, Gauss maps and duals in Real and Complex Singularities. Pitman Research Notes in Mathematics 333, pp. 148-178, 1995.

[9] J. W. Bruce, P. J. Giblin, and F. Tari. Parabolic curves of evolving surfaces. International Journal of Computer Vision, 17:291-306, 1996. 
[10] J. W. Bruce. Generic geometry and duality. LMS Lecture Note séries 201, Edited by J.P. Brasselet (1994) pp 29-60.

[11] S. Demoto. Stable maps between 2-spheres with a connected fold curve. Hiroshima Math. J. 35 (2005), 93-113.

[12] DIAS, M. D. S. S. Classificação de aplicações estáveis através do uso de grafos Dissertação de mestrado, Instituto de Ciências Matemáticas e de Computação, Universidade de São Paulo, 2012.

[13] C. M. de Jesus, D. Hacon and M. R. Fuster. Stable maps from surfaces to the plane with prescribed branching data. Topology and its Applications, (154):166-175, 2007.

[14] M. R. F. D. Hacon, C. Mendes de Jesus. Topological invariants of stable maps from a surface to the plane from a global viewpoint. Lectures Notes in Pure and Applied Mathematics, 232:227-235, 2003.

[15] M. P. do Carmo. Geometria Diferencial de Curvas e Superfícies. Coleção Textos Universitários. Sociedade Brasileira de Matemática, 2008.

[16] Y. Eliashberg. On singularities of folding type. Math. USSR-Izv., 4:1119-1134, 1970.

[17] E. L. Lima. Curso de Análise. Projeto Euclides, Rio de Janeiro: IMPA, vol. 2, 2008, pp.505-517.

[18] A. C. Felippe. Invariante global de aplicações estáveis de superfície fechada em $S^{2}$ Dissertação de mestrado, Universidade Federal de Viçosa, 2013.

[19] W. Fulton. Algebraic Topology: a First Course. Springer, 1995.

[20] T. Gaffney. The structure of LA.f, classification and an application to differential geometry. Proceedings of Symposia in Pure Maths. Vol 40 (1983), Part 1, 409-427.

[21] M. Golubitsky and V. Guillemin. Stable Mappings and Their Singularities. Number 14 in Graduated Texts in Mathematics. Springer-Verlag New York Inc., 1973. 
[22] V. Goryunov. Local invariants of mappings of surfaces into three-space. Matematisk Institut, Universitet, 1994.

[23] V. Guillemin and A. Pollack. Differential topology. Mathematics Series. Prentice-Hall, 1974.

[24] D. Hacon, C. M. de Jesus, and M. R. Fuster. Fold maps from the sphere to the plane. Experimental Maths, 15(4):491-497, 2006.

[25] D. Hacon, C. Mendes de Jesus, and M. Romero Fuster. Graphs of stable maps from closed orientable surfaces to the 2-sphere. Journal of Singularities Volume 2, 67-80, 2010.

[26] F. Harary. Graph Theory. Reading, Mass., 1969.

[27] M. Hirsch Diferential Topology., Graduate Texts in Mathematics, 33. Springer Verlag, New York, 1976.

[28] N. B. Huamaní. Grafos associados as aplicações estáveis de 3-variedades fechadas e orientadas no $\mathbb{R}^{3}$. Dissertação de mestrado, Universidade Federal de Viçosa, 2015.

[29] W. Massey. A Basic Course in Algebraic Topology. Springer, 1991.

[30] C. Mendes de Jesus, S. Moraes, and M. Romero Fuster. Stable gauss maps from a global viewpoint. Bulletin of the Brazilian Mathematical Society, New Series, 42(1):87$103,2011$.

[31] S. Jurkiewicz. Grafos - Uma Introdução. Apostila 5 do Estágio de treinamento dos alunos premiados da OBMEP, 2009.

[32] L. Kinsey. Topology of Surfaces. Springer Verlag - New York, 1994.

[33] D. S. Machado. Invariante global de aplicações estáveis de superfície fechada no plano. Dissertação de mestrado, Universidade Federal de Viçosa, 2010.

[34] Martinet, J. Deploiments versels des applications differentiables et classification des applications stables, Singularites d'Applications Differentiables, Plans-sur-Bex 1975, Springer Lecture Notes in Math. 535 (1976), 1-44. 
[35] A. Montesinos. Software superficiesII, 2009. http://www.uv.es/montesin/.

[36] J. A. Montaldi, On contact between submanifolds, Michigan Math. J. 33 (1986), 195199.

[37] T. Ohmoto. Vassiliev type invariants of order one of generic mappings from a surface to the plane. Topology of Real Singularities and Related Topics, pages 55-68, 1997.

[38] I. R. Porteous. Geometric differentiation: for the intelligence of curves and surfaces. Cambridge University Press, 2001.

[39] I. R. Porteous, The normal singularities of a submanifold, Jour. Diff. Geom., 5 (1971), 543-564.

[40] I. R. Porteous, The normal singularities of surfaces in $\mathbb{R}^{3}$, Proccedings of Symposia in Pure Mathematics, Volume 40, Part 2, (American Mathematical Society, 1983, Providence), 379-394.

[41] J. Quine. A global theorem for singularities of maps between oriented 2-manifolds. Trans. AMS, 236:307-314, 1978.

[42] F. A. T. Ohmoto. First order local invariants of apparent contours. Topology, (45):2745, 2006.

[43] J. H. Rieger. Families of maps from the plane to the plane. J. London Math. Soc. (2) 36 (1987), no. 2, 351-369.

[44] I. V. Souza. Singularidades de aplicações de Gauss estáveis. Dissertação de mestrado, Universidade Federal de Viçosa, 2012.

[45] F. Tari. Singularidades de aplicações diferenciaveis. Notas didáticas do ICMC No34, 1999.

[46] K. Tenenblat. Introdução à geometria diferencial. Ed. Univ. Brasília, 1988.

[47] L. L. T.H. Bröcker. Differentiable Germs and Catastrophes. Cambridge University Press, 1975. 
[48] J. P. Thirin, New feature points based on geometric invariantes for 3D image registration, INRIA research report No 1901, Int. J. Computer Vision, 1993.

[49] R. Thom. Structural Stability And Morphogenesis. Addison-Wesley, Advanced Book Program, 1975.

[50] R. Thom. Sur la theorie des enveloppes. Jour. Math. Pures Appl. 41 (1962), 177-192.

[51] V. Vasiliev. Complements of Discriminants of Smooth Maps: Topology and Applications. Translations of Mathematical Monographs. American Mathematical Society, 1992.

[52] A. White. Graphs, Groups and Surfaces. Mathematics Studies - Nort Holland, 1973.

[53] H. Whitney. On singularities of mappings of euclidean spaces. I. Mappings of the plane into the plane. Ann of Math, 62:374-410, 1955.

[54] T. Yamamoto and A. Kamenosono The Minimal numbers of singularities of stable maps between surfaces. Topology and its Applications 156 (2009), 2390-2405.

[55] Yung-Che. Singularity Theory and Introduction to Catastrophe. Springer, 1976. 